\title{
SubCRITICAL TRANSITION TO Turbulence in Shear Flows
}

\author{
DisSERTATION \\ FOR THE AWARD OF THE DEGREE \\ "Doctor RERUM NATURAlium" \\ of Georg-August-Universität Göttingen
}

WITHIN The DOCTORAL PROGRAM PROPhyS

of the Georg-August University School of Science (GAUSS)

\author{
SUBMITTED BY \\ LIANG SHI \\ FROM JiAngXi, ChinA
}

GötTingen, 2014 


\section{Thesis Committee}

Prof. Dr. Björn Hof

Nonlinear Dynamics and Turbulence, Institute of Science and Technology Austria

Prof. Dr. Andreas Tilgner

Institute of Geophysics, Georg-August-Universität Göttingen

\section{Member of the Examination Board}

Referee: Prof. Dr. Andreas Tilgner

Institute of Geophysics, Georg-August-Universität Göttingen

2nd Referee: Prof. Dr. Eberhard Bodenschatz

Fluid Dynamics, Pattern Formation and Biocomplexity, Max-Planck Institute for Dynamics and Self-Organization

\section{Further members of the Examination Board}

Prof. Dr. Marc Avila

Institute of Fluid Mechanics, Friedrich-Alexander-Universität Erlangen-Nürnberg

Prof. Dr. Andreas Dillman

Institute of Aerodynamics and Flow Technology, German Aerospace Center (DLR)

Prof. Dr. Björn Hof

Nonlinear Dynamics and Turbulence, Institute of Science and Technology Austria

Prof. Dr. Jörg Schumacher

Department of Mechanical Engineering, Technische Universität Ilmenau

Prof. Dr. Jürgen Vollmer

Dynamics of Complex Fluids, Max-Planck Institute for Dynamics and Self-Organization

Date of oral examination: 20th May 2014 
To Petta 



\section{ABstract}

The theme of this thesis is transition to turbulence in linearly stable shear flows. Laminar-turbulent intermittency and hysteresis are typical feature of these flows at transitional Reynolds numbers, characterizing a subcritical scenario. Subcritical transition is a dynamically rich phenomenon in nature and has been scrutinized for over a century. The critical Reynolds number for the transition and its physical mechanisms are fundamental problems in fluid dynamics and are still not fully understood. These questions are addressed in this thesis by performing direct numerical simulations of Taylor-Couette flow in linearly stable regimes: counterrotating narrow-gap regime (in the plane Couette limit) and corotating quasi-Keplerian regime. The essential contributions in this study can be summarized as follows.

In the first part of the thesis, a highly efficient parallel DNS code for turbulent Taylor-Couette flow has been developed. The Navier-Stokes equations are discretized in cylindrical coordinates with the spectral Fourier-Galerkin method in the axial and azimuthal directions, and high-order finite differences in the radial direction. Time is advanced by a second-order, semi-implicit projection scheme, which requires the solution of five Helmholtz/Poisson equations, avoids staggered grids and renders very small slip velocities. Nonlinear terms are computed with the pseudospectral method. The code is parallelized using a hybrid MPI-OpenMP strategy, which is simple to implement, reduces inter-node communications and is more efficient compared to a flat MPI parallelization. A strong scaling study shows that the hybrid code maintains very good scalability up to $3 \times 10^{4}$ processor cores and thus allows to perform simulations at higher Re with higher resolutions than previously feasible.

In the second part, this code is used to study the subcritical transition to turbulence in plane Couette flow. Here turbulent spots can grow and form continuous stripes, yet in the stripe-normal direction they remain interspersed by laminar fluid. By performing direct numerical simulations in a long narrow domain, it is observed that individual turbulent stripes are transient. In agreement with recent observations in pipe flow it is found that turbulence becomes sustained at a distinct critical point once the spatial proliferation outweighs the inherent decaying process. By resolving the asymptotic size distributions close to criticality, the feature of scale invariance at the onset of turbulence is demonstrated and the critical exponents are obtained. 
These results shows that the transition is a continuous phase transition and may belong to the directed percolation universality class.

Third, subcritical hydrodynamic turbulence is probed in Taylor-Couette flow (TCF) in the quasi-Keplerian regime at Re up to $10^{5}$. Whether hydrodynamic turbulence exists in linearly stable quasi-Keplerian flows is strongly debated and controversial results were reported. To avoid the axial end-wall effects in experiments, DNS simulations of axially periodic TCF have been conducted. By analyzing the temporal evolution of perturbation kinetic energy, secondary instability is identified and it causes the flow break down to turbulence. However, the arising turbulence eventually decays.

The effects of Earth rotation may contribute to the observed turbulence in experiments. In the final part of the thesis, linear stability and transient energy growth have been studied in the plane Couette flow with system rotation perpendicular to the wall. It is found that wall-normal external system rotation causes linear instability. At small rotation rates, the onset of linear instability scales inversely with the rotation rate and the optimal transient growth in the linearly stable region is slightly enhanced, $\sim \operatorname{Re}^{2}$. At large rotation rates, the transient growth is significantly inhibited. 


\section{ACKNOWLEDGMENTS}

This thesis is more than to award a "Dr." title. There are stories of people, with or without or half "Dr.". This thesis is more than a sum of letters, numbers and figures. There are efforts from people, valuable or very valuable or very very valuable.

Here I would like to express my most sincere appreciation to

Prof. Björn Hof. His supervision with great scientific vision and meticulous care makes this thesis a success. Björn always gives me the strongest support whenever I need, always allows me to do whatever satisfies my curiosity. Besides the scientific aspects, during my stay in Björn's lab, some critical bad (also good) habitudes and personality traits have been discovered, which I will never forget about and will try the best to get over.

Prof. Marc Avila. The hand-in-hand working experience with Marc is so precious. The humour and the laugh that Marc brought to me and to the group will never be forgotten. Encouraging students and thinking in the students' place are enormously appreciated. His critics and wisdom make this thesis much much better.

Prof. Andreas Tilgner. His solid theoretical knowledge on fluid mechanics and rich numerical experiences guided me quickly through the difficulties that I encountered in the Ekman-Couette project. The opportunity offered by him to study the astrophysical flows and to experience the German collaborative research culture will be certainly very beneficial to my future career.

Dr. Markus Rampp. Collaboration with Markus on the hybrid code "nsCouette" constitutes an important part of my $\mathrm{PhD}$ time. His impressive technical acume and competency on IT and HPC help to accelerate "nsCouette" significantly. Without the excellent technical support from Markus for my DNS simulations, my thesis could not go so smoothly.

the members in the family group of Björn: Dr. Sebastian Altmeyer (Sleepless nation), Dr. Alberto de Lozar (Spanish), Dr. Markus Holzner (Italian), Dr. 
Shreyas Vaman Jalikop (Indian), Dr. Jakob Kühnen (Austrian), Dr. Grégoire Lemoult (French), Simon Maretzke (Genius nation), Dr. Jose Manuel Gallardo Ruiz (Spanish), Dr. Nicolas Roland (French), Baofang Song (Chinese), Dr. Devranjan Samanta (Indian), Jan Schlüter (German), Dr. Paul Steffen (Haha nation), Xiaoyun Tu (Mama nation), Dr. Mukund Vasudevan (Indian), Dr. Duo $\mathrm{Xu}$ (Chinese). What an international group! Diversity creates NATURE and SCIENCE!

Dr. Xing Wei from Princeton and Hannes Brauckmann from Marburg. Discussions with them are joyful and valuable.

my friend Xiguang Zhang and the "eating" group: Li Li, Wei Huang, De Zhou, Xu Tian, Hui Wang, Xiaowei Wang and others. Eating, playing and traveling are the theme; friendship is the precipitate.

Max Planck Institute for Dynamics and Self-Organization, Georg-August-Universität Göttingen (DFG SFB 963) and Institute of Science and Technology Austria. Money is a necessary goods for everyone.

many supercomputer clusters: Rechnungszentrum Garching, Juropa, PRACE, GWDG. Core-hours to computational scientists are like air to human being.

, last but not least, my family and Petta, for eternal love.

"Extra Gottingam NON EST VITA, Si EST VITA NON EST ITA"

("Outside of Göttingen there's no life; if there's a life, none like this") 
Abstract i

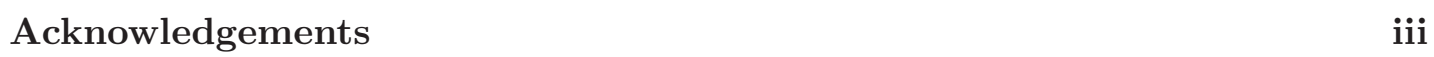

List of Figures vii

List of Tables ix

Abbreviations $\quad$ xi

Symbols $\quad$ xiii

$\begin{array}{llr}1 & \text { Introduction } & 1\end{array}$

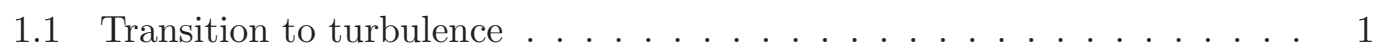

1.2 Subject of the thesis . . . . . . . . . . . . . . . . . 3

1.3 Threshold of sustained turbulence in PCF $\ldots \ldots \ldots \ldots$

1.4 Stability of rotating flows in astrophysical accretion disks . . . . . . . 9 9

1.5 Thesis outline . . . . . . . . . . . . . . . . . . . . . 12

2 A highly efficient parallel DNS code nsCouette $\quad 15$

2.1 Introduction . . . . . . . . . . . . . . . . . . . . . . . . 15

2.2 Navier-Stokes equations $\ldots \ldots \ldots \ldots$. . . . . . . . . . . . . . 17

2.3 Numerical method $\ldots \ldots \ldots \ldots \ldots$

2.3 .1 Spatial discretization . . . . . . . . . . . . . . . . 18

2.3 .2 Temporal scheme . . . . . . . . . . . . . . . . . . . . 20

2.4 Parallelization scheme and its implementation . . . . . . . . . . . 21

2.5 Numerical Accuracy and Code Validation . . . . . . . . . . . 23

2.5 .1 Laminar flow . . . . . . . . . . . . . . . . . . 23

2.5 .2 Time-dependent flow and slip velocity at walls . . . . . . . 24

2.5 .3 Localized turbulence at moderate Re . . . . . . . . . . . 27

2.5 .4 Turbulent flow at high $\mathrm{Re} \ldots \ldots$. . . . . . . . . . . 27

2.6 Computational efficiency . . . . . . . . . . . . . . . . . . . . . . . . . . . . . . 30

2.6 .1 Benchmark setup . . . . . . . . . . . . . . . . . . 30

2.6 .2 Benchmark results and discussion . . . . . . . . . . . . . 31

2.7 Discussion and summary . . . . . . . . . . . . . . . . . 33

\begin{tabular}{|lll}
3 & Onset of sustained turbulence in Couette flow & 35
\end{tabular}

3.1 Introduction . . . . . . . . . . . . . . . . . 35 
3.2 Numerical experiments . . . . . . . . . . . . . . . . . . . . . . 36

3.3 Memoryless dynamical processes $\ldots \ldots \ldots$. . . . . . . . . . . . 37

3.4 Threshold of sustained turbulence . . . . . . . . . . . . . . . 40

$3.5 \quad$ Scale invariance at criticality $\ldots \ldots \ldots$. . . . . . . . . . . . . 40

3.6 Critical exponents at criticality . . . . . . . . . . . . . . . . . . 42

3.7 Discussion and summary . . . . . . . . . . . . . . . . . . . . 47

$4 \quad$ Nonlinear transition to turbulence in quasi-Keplerian flow $\quad 49$

4.1 Numerical specification . . . . . . . . . . . . . . . . 50

4.2 Numerical results . . . . . . . . . . . . . . . . . . . . . . . 52

4.2 .1 Secondary instability . . . . . . . . . . . . . . . . . 54

4.2 .2 Decaving turbulence $\ldots \ldots \ldots \ldots \ldots$

4.3 Discussion and summary . . . . . . . . . . . . . . . . . 56

\begin{tabular}{|lll}
5 & Transient growth of Couette flow under Coriolis force & 61
\end{tabular}

5.1 Introduction . . . . . . . . . . . . . . . . . . 61

5.2 Problem formulation . . . . . . . . . . . . . . . . . . . 63

5.3 Linear instability . . . . . . . . . . . . . . . . . . . . . . 65

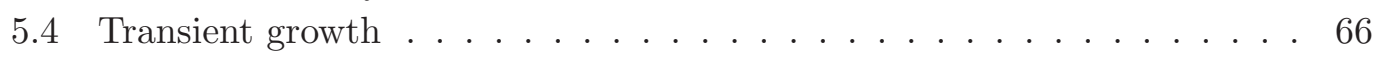

5.5 Discussion and summary . . . . . . . . . . . . . . . . . 69

$\begin{array}{lll}6 & \text { Conclusions } & 73\end{array}$

6.1 Results and discussion . . . . . . . . . . . . . . . . . . . 74

6.2 Future work . . . . . . . . . . . . . . . . . . 76

\begin{tabular}{|lll}
\hline A Computing the azimuthal wave speed of wavy vortices in TCF & 77
\end{tabular}

\begin{tabular}{|lll}
\hline Onset of sustained turbulence in less-resolved Couette flow & 81
\end{tabular}

B.1 Steady-state experiments . . . . . . . . . . . . . . . . 8 81

B.2 Quenching experiments . . . . . . . . . . . . . . . . . . . . 84

B.3 Discussion and conclusion . . . . . . . . . . . . . . . 84

\begin{tabular}{ll}
\hline C Base velocity profile in Ekman-Couette flow & 87
\end{tabular}

\begin{tabular}{lr}
\hline D Publications & 89
\end{tabular}

\begin{tabular}{ll}
\hline Bibliography & 91
\end{tabular} 


\section{List OF FigURES}

1.1 Cartoon of supercritical and subcritical path towards turbulence . . . . 2

1.2 Schematic of Taylor-Couette flow and its parameter space . . . . . . . . 5

1.3 Bond directed percolation model. . . . . . . . . . . . . . . 7

$1.4 \quad$ Spatial-temporal dynamics in bond directed percolation. . . . . . . . . . 7

1.5 Artist rendering of astrophysical accretion disk . . . . . . . . . . 10

2.1 Schematic of the global matrix transposition MPI_Alltoall() . . . . . 22

2.2 Laminar Couette flow . . . . . . . . . . . . . . . . . . 24

2.3 The global relative error $\mathcal{E}_{2} \ldots \ldots \ldots \ldots \ldots \ldots$

2.4 Taylor vortices . . . . . . . . . . . . . . . . . . . . . . . 25

2.5 Wavy Tavlor vortices . . . . . . . . . . . . . . . . . . . . . 26

2.6 Normalized velocity slip at the inner cylinder . . . . . . . . . . . 27

2.7 Probability distributions of the splitting time of single turbulent stripe . 28

2.8 Quasi-Nusselt number at fully turbulent state . . . . . . . . . . . . . 29

2.9 Isosurfaces of the streamwise vorticity in the quasi-Keplerian regime . . 30

2.10 Scalability of nsCouette . . . . . . . . . . . . . . . 32

3.1 Growth of a turbulent spot in PCF . . . . . . . . . . . . . 36

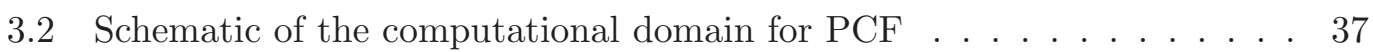

3.3 Numerical resolution . . . . . . . . . . . . . . . . . . . . . . . 38

3.4 Probability distributions of single turbulent stripes . . . . . . . . . . . . . 39

$3.5 \quad$ Scaling of the mean lifetime and splitting time with $\mathrm{Re} . \ldots . . . . .40$

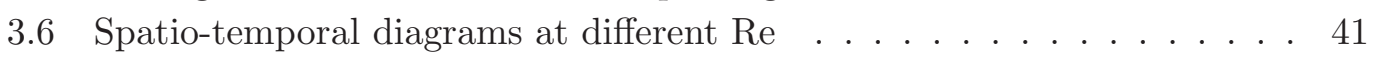

$3.7 \quad$ Size distributions of laminar gaps at $\mathrm{Re}=329$ and $\mathrm{Re}=340$. . . . . . 42

3.8 Temporal evolution of the ensemble-averaged turbulent fraction . . . . . 43

3.9 Mean turbulent fraction as a function of Re. . . . . . . . . . . . . . . . 44

3.10 PDF of laminar gap sizes at different Rd . . . . . . . . . . . . . . . . . . 45

3.11 Probability distribution of the stripe widths $\ldots \ldots$. . . . . . . . 46

3.12 Mean turbulent width as a function of $\mathrm{Re} \ldots \ldots . . \ldots 46$

4.1 The parameter space $R e_{\mathrm{s}}-R_{0} \ldots \ldots \ldots \ldots \ldots$. . . . . . . . . . . 50

$4.2 \quad$ Energy spectra $E^{l, n}(k) \ldots \ldots \ldots \ldots \ldots \ldots \ldots \ldots$

4.3 Temporal evolution of the normalized perturbation energy . . . . . . . . 53

4.4 Temporal evolution of the axial modal energy . . . . . . . . . . . . 55

4.5 Axial and Azimuthal power and dissipation spectra . . . . . . . . . 55

4.6 Temporal evolution of the velocity $\ldots \ldots \ldots$. . . . . . . . . . . 58

4.7 Temporal evolution of the modal energy at $R e_{i}=1 \times 10^{5} \ldots \ldots$. . . . 59

$4.82 \mathrm{D}$ contour plot of the streamwise vorticity $\omega_{\theta} \ldots \ldots \ldots$. . . . . . 60 
$4.93 \mathrm{D}$ contour plot of the streamwise vorticity $\omega_{\theta} \ldots \ldots \ldots$. . . . . . . 60

5.1 Schematic of Ekman-Couette flow . . . . . . . . . . . . . . . 62

5.2 Base velocity profiles of rotating PCE . . . . . . . . . . . . 64

5.3 Critical Reynolds number $\operatorname{Re}^{c}$ as a function of $\Omega \ldots \ldots$. . . . . . . 66

5.4 The variation with $\Omega$ of the wavenumber $k$ and the angle $\theta$. . . . . 66

5.5 Transient growth and Convergency study . . . . . . . . . . . 67

5.6 Contour plot in $(\alpha, \beta)$ space of the global maximum growth rate $G^{m}$. . 68

5.7 Contour plot of the global optimal growth $G^{\text {opt }}$ in the $\Omega$-Re plane . . . . 69

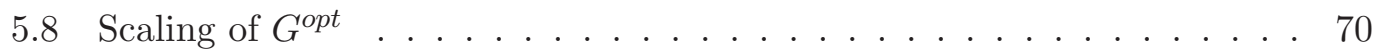

5.9 Scaling with Reynolds number at $\Omega=0$ and $\Omega=0.05$. . . . . . . . 71

5.10 Contour plot of the optimal perturbation $v^{\text {opt }}(x, y, z=0) \ldots 71$

A.1 Time series of the velocity at a mid-gap point . . . . . . . . . . . . 78

A.2 The 2-norm of the velocity difference $\mathcal{E}$. . . . . . . . . . . . . . 79

B.1 Temporal evolution of the turbulent fraction at different Re . . . . . . 82

B.2 Mean turbulent fraction $\bar{F}_{T}$ against $R \theta \ldots$. . . . . . . . . . . . . 83

B.3 Power density function of laminar gap sizes . . . . . . . . . . . . . . . . 83

B.4 Turbulent fraction in the quenching experiments . . . . . . . . . . . 84

B.5 Rescaled turbulent fraction in the quenching experiments . . . . . 85

B.6 Scaling of mean turbulent fraction in fully- and less-resolved simulations 86

B.7 Influence of the cutoff value. . . . . . . . . . . . . . . . . . . 86 


\section{List OF TABLES}

1.1 The universal DP exponents . . . . . . . . . . . . . . . . . . 8

2.1 Critical Reynolds number for Tavlor vortices . . . . . . . . . . . . . . 25

2.2 Breakdown of Runtime and parallel efficient . . . . . . . . . . . . . . . 33

3.1 The Re-dependent $L_{z}$ and resolutions . . . . . . . . . . . . 43

3.2 The power-law fits of the mean turbulent fractions . . . . . . . . . . . . 44

3.3 The best power fits of the mean turbulent fractions . . . . . . . . . . 45

4.1 Parameter comparison between astrophysical Keplerian flows and TCF . 51

4.2 Numerical parameters in TCF simulations . . . . . . . . . . . . . . 52

4.3 Transient growth rates of the optimal perturbations . . . . . . . . . 52

4.4 A comparison between our DNS simulations and the ones from Twente. 56

5.1 Existing experimental setups of plane Couette flows . . . . . . . . . . 72 



\section{ABbreviations}

$\begin{array}{ll}\text { NS } & \text { Navier-Stokes } \\ \text { PCF } & \text { plane Couette flow } \\ \text { TCF } & \text { Taylor-Couette flow } \\ \text { DNS } & \text { direct numerical simulation } \\ \text { DP } & \text { directed percolation } \\ \text { STI } & \text { Spatio-temporal intermittency } \\ \text { FD } & \text { finite difference } \\ \text { HPC } & \text { high performance computing } \\ \text { MPI } & \text { massage passing interface } \\ \text { OpenMP } & \text { open multiprocessing } \\ \text { CFL } & \text { Courant Friedrichs Lewy }\end{array}$





\section{Symbols}

$\begin{array}{lll}R e & \text { Reynolds number } & - \\ R e & \text { Reynolds number of inner cylinder } & - \\ R e & \text { Reynolds number of outer cylinder } & - \\ R_{\Omega} & \text { Rotation number } & - \\ R o & \text { Rossby number } & - \\ r_{i} & \text { radius of the inner cylinder } & - \\ r_{o} & \text { radius of the outer cylinder } & - \\ d & \text { gap distance } & m \\ h & \text { half gap distance } & m \\ U, V, W & \text { velocity } & \mathrm{m} \cdot \mathrm{s}^{-1} \\ k & \text { wavenumber } & 1 / \mathrm{m} \\ E & \text { kinetic energy } & - \\ & & \\ \omega & \text { angular velocity } & \mathrm{rads} \cdot \mathrm{s}^{-1} \\ \eta & \text { radius ratio } r_{i} / r_{o} & - \\ \Omega & \text { Rotation number } & - \\ \nu & \text { kinetic viscosity } & \mathrm{m}^{2} \cdot s \\ \tau_{h} & \text { advection time unit } & \mathrm{s} \\ \tau_{\nu} & \text { viscous time unit } & \mathrm{s} \\ \theta & \text { angle } & \mathrm{rads}\end{array}$



"A JOURNEY OF A THOUSAND MILES STARTS With A

SINGLE STEP."

LAOZI (C. $400-500$ BC)

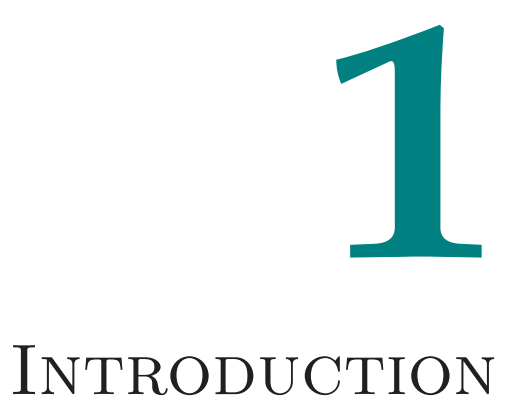

\subsection{Transition to turbulence}

In fluid dynamics, laminar-turbulent transition, the process of a laminar flow becoming turbulent, has been the subject of research for over a century. This transition arises throughout nature from star formation, weather dynamics, down to cardiovascular circulation. A typical example is a rising plume of smoke from a cigarette, where the regular, non-mixing, smooth motions of smoke at the beginning turns chaotic, disordered and turbulent at some distance above the cigarette. The study of laminarturbulent transition has significant theoretical meaning, to understand the origin and nature of turbulence, which is described by Nobel Laureate Richard Feymann as "the most important unsolved problem of classical physics". Besides this, turbulence transition appears also frequently in engineering and has both detrimental and beneficial effects. Turbulent flows lead to much higher energy losses during the transport of liquid due to high wall friction, whereas turbulence improves drastically the mixing efficiency in chemical reactions. If the transition mechanisms were fully understood, efficient turbulence-controlling strategies could be conceived to either delay or promote a transition.

The first quantitative study on transition to turbulent flow dates back to the cir-

cular pipe experiments performed by Osborne Reynolds in 1883 [1]. In his study, Reynolds tried to find a critical velocity below which "the disturbance would settle 
down into a steady condition" and above which turbulence persists. However, he realized that the seemingly simple experiments were extremely complicated. Reynolds finally couldn't obtain a precise critical value but concluded with a rough but relatively accurate estimation. In this paper Reynolds introduced the idea of the most important non-dimensional number in fluid mechanics, later named after him as the Reynolds number, $R e=\frac{U L}{\nu}$, where $L$ is the length scale upon which the characteristic velocity $U$ varies and $\nu$ is the kinematic viscosity . The puzzle left behind by Reynolds triggered tremendous research work on the subject of flow instabilities, including Rayleigh [2], Taylor [3, 4], Laudau [5], Rotta [6], Lorenz [7], Coles [8], Ruelle and Takens [9], Swinney and Gollub [10], Pomeau [11]. However, it was not until 2011 upon cumulative efforts of previous experiments and numerical simulations that the puzzle of the critical point in pipe flow was finally resolved [12]. The whole exciting story of turbulence transition in pipe flow has been recently documented in Kerstin Avila's PhD thesis [13].
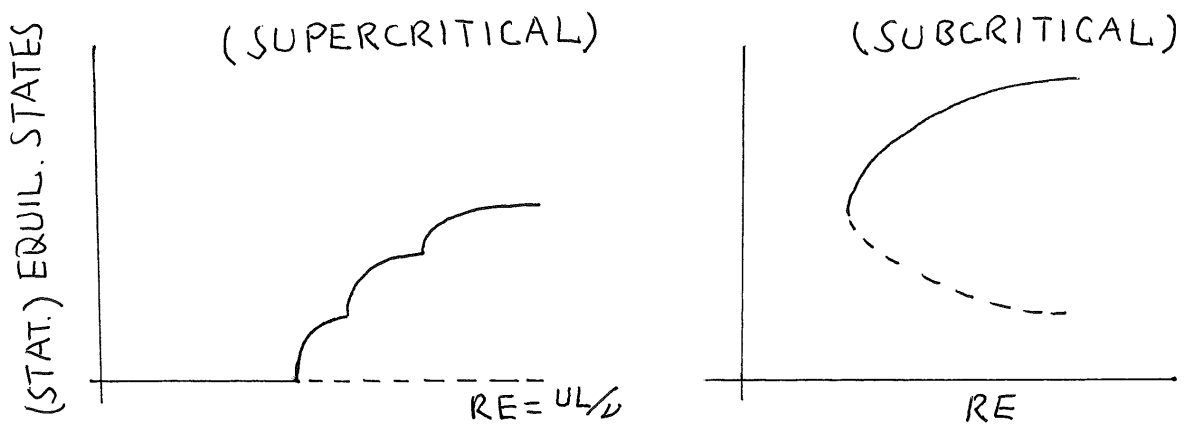

Figure 1.1: Cartoon illustrating the supercritical and subcritical transition to turbulence: statistical equilibrium (Stat. Equil.) states versus the control parameter Reynolds number $R e$. The solid and dashed lines denotes the stable and unstable equilibrium states, respectively. Supercritical transition is characterized by bifurcations sequences and continuous variation, whereas subcritical transition has feature of discontinuity and hysteresis as $R e$ varies.

The transition scenarios from laminar to turbulent flow can be, in light of the nonlinear dynamics theory, categorized into two types: supercritical and subcritical transition. The schematic pictures are illustrated in Fig. 1.1. Supercritical transition often undergoes a series of successive bifurcations and instabilities before the flow eventually breaks down to turbulence (Fig. 1.1 (left)). One of the typical examples is Taylor-Couette flow (TCF) with stationary outer cylinder. TCF is the flow between two independently rotating concentric cylinders. When the outer cylinder is fixed and the inner cylinder is rotated, the base velocity profile (an equilibrium solution of the governing equations of the incompressible fluid flows, Navier-Stokes equations) is linearly unstable according to Rayleigh's criterion [2]. Rayleigh's criterion derives from an inviscid analysis and states that flow with outward decreasing angular momentum is linearly unstable. However, the flow maintains the laminar motion at small Re due to the stabilizing effect of viscosity. Beyond a certain critical Reynolds number, a new state bifurcates from the 
laminar one and it is characterized by axially-periodic roll structures, known as Taylor vortices. As Re is further increased, new (statistically) steady states arise and the complexity of the flow augments continuously, starting from wavy vortices, modulated wavy vortices, to turbulent Taylor vortices and finally cascading to turbulence [14].

In contrast, subcritical transition is much more complicated and has been puzzling many researchers, in that it is very sensitive to the initial conditions and to the historical path of the flow. In this case, the laminar flow is linearly stable. With perturbations of finite amplitude, the complexity of the flow displays discontinuous feature as Re varies: Turbulence arises or decays abruptly, as shown in Fig. 1.1 (right). What makes things more delicate is the fact that turbulent and laminar regions can coexist in the system at moderate $R e$. Pipe and plane Couette flow (PCF) exemplify excellently this type of transition. Literature on the subcritical transition to turbulence in pipe flow can be found in [15 20]. PCF is an idealized flow which denotes the fluid flow sheared between two parallel infinite plates at a finite distance apart and moving with different speed. The flow is purely driven by the viscous force of the fluid and the shear formed by the plates. The linear velocity profile of the base flow in PCF has been theoretically proven to be linearly stable [21, 22]. However, by sufficiently perturbing the flow, turbulence is observed in both experiments [23] and numerical simulations [24]. At moderate Re turbulence forms localized structures, such as turbulent spots and stripes, surrounded by laminar regions. The observation of this spatial irregularity and complexity has actually raised a wave of investigations and it is a hot topic in the community of fluid dynamics. More significantly, it shifts the physical understanding of the transition process from the traditional viewpoints of consecutive bifurcations [5] or temporal chaos 9, 10, 25] to the spatio-temporal perspectives [26]. As has been shown in pipe flow [12] and as will be further evidenced in the first part of this thesis, the spatial complex dynamics indeed plays a crucial role in the subcritical transitions process towards turbulence.

\subsection{Subject of the thesis}

In this thesis, direct numerical simulations (DNS) were performed to study the subcritical transition to turbulence in shear flows. Distinguished from the Reynolds-averaged Navier-Stokes (RANS) and large-eddy simulations (LES), DNS solves directly the Navier-Stokes equations and resolves the whole range of scales relevant to the turbulence dynamics, hence providing data of high fidelity. Moreover, DNS enables access to the whole-field data of physical quantities and it is free from the unavoidable defects in lab experiments (boundary roughness, environmental noise, etc). The disadvantage of DNS is that it is computationally very costly, since the relevant spatial and temporal scales span several orders of magnitude. The largest scale is about the domain 
size $L$ while the smallest one $l$, according Kolmogolov's theory [27, 28], is set by the energy dissipation rate $\epsilon$ and the viscosity $\nu, l \sim\left(\nu^{3} / \epsilon\right)^{1 / 4}$. Assuming that the energycontaining scales dominate the dissipation rate $\epsilon \sim u^{3} / L$, the scale separation $L / l$ is thus enlarged with the increasing Reynolds number as $L / l \sim(R e)^{3 / 4}$. Therefore, the total complexity of a DNS for a three dimensional unsteady flow amounts up to $\mathcal{O}\left(R e^{3}\right)$, with a temporal duration of order $\sim \mathcal{O}\left(R e^{3 / 4}\right)$ needed for the flow to cross the computational domain [29]. One goal of this thesis is to develop a highly efficient parallel DNS code which allows us to make full advantage of modern supercomputers and then to conduct cutting-edge research on long-standing classical problems in transition to turbulence.

The flow between two independently rotating concentric cylinders, Taylor-Couette flow (TCF), is the main subject of our study. Fig. 1.2 (left) shows the geometry of the flow. The inner (outer) cylinder has radius $r_{i}\left(r_{o}\right)$ and rotates at a speed of $\Omega_{i}\left(\Omega_{o}\right)$. The Reynolds number in the inner and outer cylinder is defined as $R e_{i, o}=\Omega_{i, o} r_{i, o} d / \nu$, where $d=r_{o}-r_{i}$ is the gap between the cylinders. The geometry of TCF is fully specified by two dimensionless parameters: the radii-ratio $\eta=r_{i} / r_{o}$ and the length-togap aspect-ratio $\Gamma=L_{z} / d$, where $L_{z}$ is the axial length of the cylinders. The laminar base flow, often called circular Couette flow, has only azimuthal velocity component. The amplitude of the angular velocity $\Omega$ depends on the location in radial direction, as

$$
\Omega^{b}(r)=C_{1}+\frac{C_{2}}{r^{2}}, \text { with } C_{1}=\frac{R e_{o}-\eta R e_{i}}{1+\eta}, C_{2}=\frac{\eta\left(R e_{i}-\eta R e_{o}\right)}{(1-\eta)\left(1-\eta^{2}\right)},
$$

which corresponds to a pure rotary shear flow.

Two different regimes in TC flow are considered in the thesis: counter-rotating regime in the narrow-gap limit (Regime I) and co-rotating quasi-Keplerian regime (Regime II), as shown in Fig. 1.2 (right). In the former, the radius ratio is $\eta=0.993$, where the curvature effects are negligible and the flow behaviors asymptotically as plane Couette flow (PCF). PCF is a canonical example for studying shear flow instabilities and subcritical transition to turbulence. The latter one (Regime II) denotes the corotating region below the Rayleigh line and above the solid-body line in the $\left(R e_{o}, R e_{i}\right)$ parameter space. The Rayleigh line $\left(R e_{o}=\eta R e_{i}\right)$ seperates the linearly stable and unstable flows for inviscid fluids. Below the Rayleigh line, the circular couette flow is linearly stable. On the solid-body line the fluids behaves like a rigid body without shear, which means $\Omega_{i}=\Omega_{o}$ or $R e_{i}=\eta R e_{o}$. In the region limited by these two lines, the base velocity profiles satisfy two conditions: (1) radially increasing angular momentum $\frac{d \Omega^{b}(r) r^{2}}{d r}>0 ;(2)$ radially decreasing angular velocity $\frac{d \Omega^{b}(r)}{d r}<0$. Since the astrophysical Keplerian velocity $\Omega^{K}(r) \sim r^{-3 / 2}$ satisfies both conditions, the region in light blue is often called quasi-Keplerian regime, especially in studying the astrophysical Keplerian flows. 


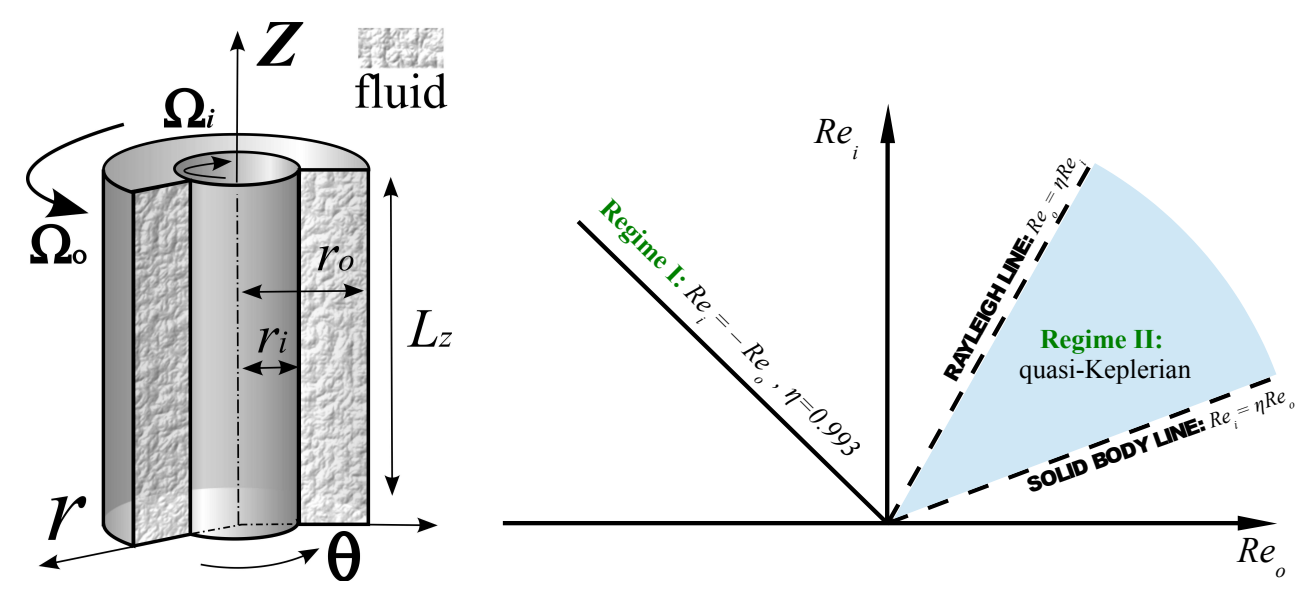

Figure 1.2: (Left) Schematic of the Taylor-Couette system in cylindrical coordinates. The inner and outer cylinder rotate independently with speeds $\Omega_{i}$ and $\Omega_{o}$, respectively. No-slip boundary conditions at the cylinder are used together with axially periodic boundary conditions. The fluid between the cylinders (hatched region) flows by the shear force due to the fluid viscosity. (Right) The parameter space $R e_{o}-R e_{i}$. Two different regimes are here considered: exactly counter-rotating regime I and corotating quasi-Keplerian regime II. In regime I, we take $R e_{i}=-R e_{o}$ and $\eta=0.993$, while the quasi-Keplerian regime is bounded by the Rayleigh line and the solid-body line.

There are two scientific problems that are concerned in this thesis. The first one is on the critical Reynolds number beyond which turbulence sustains and on the physical nature of such a transition in PCF. The simulations were performed in flow regime I in TCF. As shown in an earlier study [30] TCF approaches the PCF limit for these parameter settings and is linearly stable for all Reynolds numbers relevant in this thesis. The second problem is to check whether turbulence in the flow regime II can arise via subcritical mechanisms. This study is motivated by the accretion in weaklyionized astrophysical disks, where the accretion process requires angular momentum transport in the disk and turbulence-enhanced viscosity is the mechanism thought to be responsible for the transportation. However, the question how turbulence arises in cold disks is still unclear. Subcritical transition to turbulence (like in other shear flows) may be a potential candidate.

\subsection{Threshold of sustained turbulence in PCF}

The critical Reynolds number for sustained turbulence in PCF has been investigated for a long time. The classical theoretical approaches to study the stability of PCF include the Kelvin modes method [31], linear stability analysis [32, 33] and the method of solving the initial-value problem of the inviscid PCF introduced by Case [34]. All those methods failed to predict a critical Re since the laminar profile of PCF has been mathematically proven to be stable to infinitesimal perturbations (or to be linearly 
stable) for all Re [21]. On the other hand, Ellingsen and Palm [35] showed that finite three-dimensional disturbances may lead to breakdown of the laminar motion, which was supported by Nagata's discovery of 3D finite-amplitude exact solutions for $R e>125$, giving a lower bound for the onset of turbulence in PCF [36]. Through the numerical investigation of the development of localized turbulent spots, Lundbladh and Johansson [24] proposed for the first time an estimation of the critical Reynolds number $R e^{c} \simeq 375$, which agreed well with the experimental results $R e^{c}=360 \pm 10$ obtained one year later by Tillmark and Alfredsson [23]. These work motivated an effort from the French CEA Saclay group to reveal the subcritical transition to turbulence in PCF. The critical Re had also been successively refined by employing different methods, from examining the evolution of turbulent spots $\left(R e^{c}=370 \pm 10\right)$ [37], measuring the critical amplitude of the initial perturbation to trigger turbulence $\left(R e^{c}=325 \pm 5\right)$ [38], to the statistical approach of measuring the mean lifetime of turbulent spots $\left(R e^{c}=\right.$ $323 \pm 2$ ) [39]. With this statistical method Bottin and Chaté thought "the transition to turbulence in plane Couette flow is best studied".

With the fast development of supercomputing infrastructures and parallel computing software, DNS becomes another powerful tool to explore the physics of turbulence (see [29, 40 42]). By performing DNS simulations over a computational domain with similar size as in previous experiments [39], Duguet et al. [43] monitored the velocity fluctuations and found that the threshold of sustained turbulence happens at $R e_{c}=$ $324 \pm 1$. However, these results have been contradicted by the finding of Schneider et. al. [44], who showed in a small domain of PCF that turbulence is always transient, as argued earlier in pipe flow [16, 45]. Interestingly, it is observed in previous studies on linearly stable flows that at moderate Re turbulence firstly appears in localized form, such as turbulent "puffs" in a pipe [46] or turbulent "spots" or "bands" in PCF and TCF $[8,24,47,48]$, before eventually evolving into fully developed turbulence at higher Re. The dynamics of localized turbulence are rather complex: decay, spreading, splitting, merging, etc. The transient nature of localized turbulence in small systems in [44] (without sufficient spatial interactions) does not necessarily indicate the absence of sustained turbulence in a spatially extended system. This thesis actually shows that the spatial dynamics of the temporally transient localized turbulence lead to sustained turbulence in PCF.

\section{Continuous or discontinuous phase transition?}

Meanwhile, extensive work from French scientists has been devoted to understand the nature of subcritical transition to turbulence in PCF from the viewpoint of phase transition in statistical physics, following the line of argument by Pomeau [11]. Pomeau proposed that subcritical turbulence bears resemblance to "directed percolation" (DP) in statistical physics. Percolation generally describes the physical phenomenon of a 
fluid spreading randomly through a medium, e.g., the wetting of a porous medium, and was firstly mathematically studied by Broadbent and Hammersley [49]. Here the spreading of the fluid is determined by the permeability or randomness of the medium instead of the randomness of the fluid itself like in diffusion processes. When the symmetry of the system is broken in a particular direction due to an external force or law (gravity, time, etc), percolation is usually constrained in this direction and therefore called DP. DP problem was introduced by S. P. Obukhov [50] and the aforementioned wetting problem under gravity is an example. Nowadays, the term DP mostly refers to a universality class of continuous phase transitions out of thermodynamic equilibrium. Taking the simplest bond DP model for example (Fig. 1.3), each active (blue) site at the current level connects to the neighboring two sites at one level down with a fixed probability $p$. This probability controls the local connectivity and is the only parameter to determine the global permeability of the system. Beyond some critical threshold $p>p_{c}$, the system undergoes a phase transition from impermeable to permeable, as shown in Fig. 1.4,

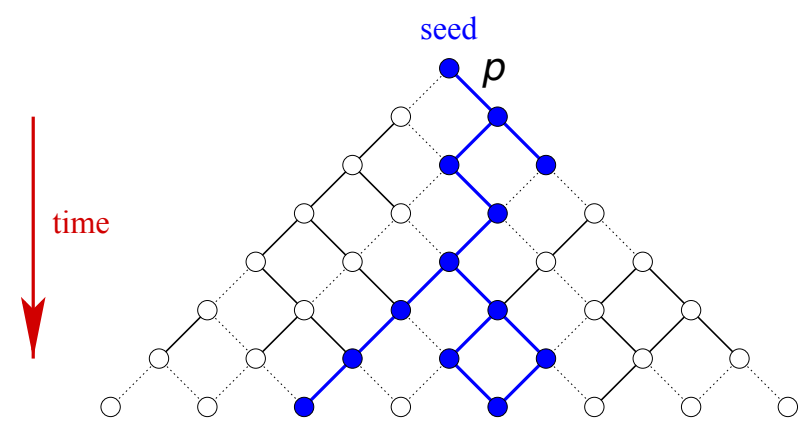

FiguRE 1.3: Bond directed percolation model 51]. In this model, each active site (in blue) connects to a neighbor with probability of $p$. As time evolves downwards, whether the initial active site reaches the bottom totally depends on $p$.
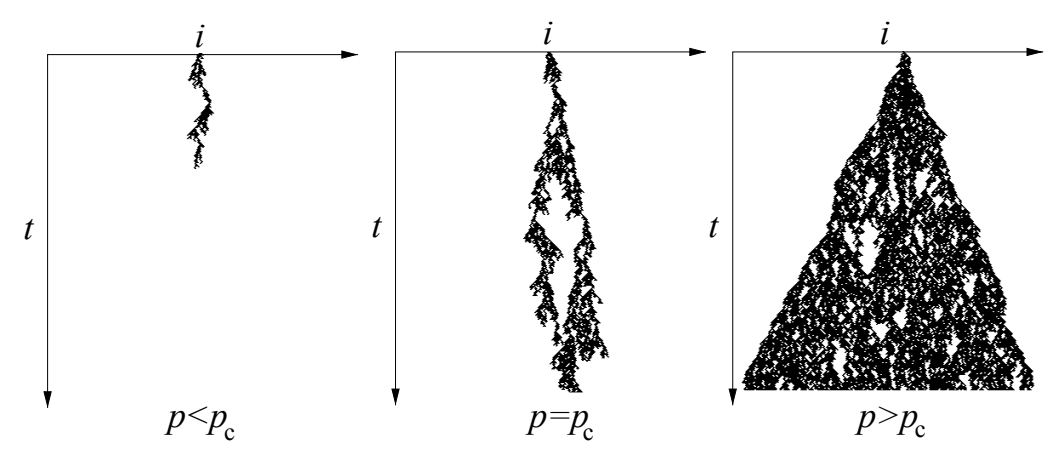

Figure 1.4: Spatial-temporal dynamics in bond DP model [51]. Three different scenarios are shown: below the critical value $p^{c}$, the initial active sites simply die away, while at the critical value they struggle to reach the end. When $p>p^{c}$, the system is almost fully active. These diagrams are typical for systems in the DP universality class. 
The connection proposed by Pomeau between subcritical turbulence and DP model seems reasonable at least at a qualitative level: the localized turbulence can be interpreted as the active individual site, whereas the laminar flow as the inactive site; The Reynolds number Re plays the role of the connecting probability $p$. This analogy had been further advocated by Chaté and Manneville [26], who observed typical features of critical phenomena by numerically solving a group of partial differential equations. It is worth to note that this paper emphasised for the first time both the spatial and temporal aspects in the transition to turbulence with its conceptually beautiful title "Transition to Turbulence via Spatiotemporal Intermittency", although the concept "spatiotemporal intermittency" (STI) was coined earlier in a paper by Kaneko [52]. Additionally, Janssen [53] and Grassberger [54] earlier conjectured that an absorbing continuous phase transition with a single non-negative order parameter (density of active sites in bond DP model), short-range interactions and without unconventional symmetries or quenched disorder generally falls into the DP universality class. The transition to subcritical turbulence appears to fulfill all the above requirements:

1) linearly stable laminar flow;

2) non-negative turbulent fraction as the only order parameter;

3) no long-range interactions ${ }^{1}$.

Quantitatively, the question whether transition via STI to subcritical turbulence falls into the DP universality class, according to Pomeau [11], "could be checked, at least in principle by comparing the predicted "universal" critical exponents of directed percolation with experimental data." In the DP universality class, the order parameter $\rho$, the spatial and the temporal correlation length $\left(\xi_{\perp}\right.$ and $\left.\xi_{\|}\right)$follow universal power-law scalings with a universal exponent, as listed in Table 1.1. To the best of my knowledge, these universal scaling behaviors were only obtained from theoretical and model studies and there is no experimental or numerical evidences, except the one realized in liquid crystals by Takeuchi et al. [55]. In NS equations it has not been shown yet.

\begin{tabular}{cccc}
\hline \hline & order parameter & spatial & temporal \\
\hline scaling & $\rho \sim\left(p-p_{c}\right)^{\beta}$ & $\xi_{\perp} \sim\left(p-p_{c}\right)^{\nu_{\perp}}$ & $\xi_{\|} \sim\left(p-p_{c}\right)^{\nu_{\|}}$ \\
exponents & $\beta$ & $\nu_{\perp}$ & $\nu_{\|}$ \\
\hline 1D & 0.276 & 1.097 & 1.734 \\
2D & 0.583 & 0.733 & 1.295 \\
\hline \hline
\end{tabular}

TABLE 1.1: The universal critical exponents in 1D and 2D DP universality class [56].

\footnotetext{
${ }^{1}$ Although pressure is a global quantity in the flow, the dynamics of localized turbulence (spots or stripes) is confined to the nearby region.
} 
Through a model study in coupled map lattices, Chaté and Manneville [57] quantitatively investigated the STI phenomenon and statistically measured the scaling behaviors. They found a continuous phase transition but with a different exponent and thus concluded that STI does not belong to the DP universality class. Careful readers will notice that the results in this paper actually do not provide sufficient evidence to support this conclusion. The only obvious deviation from DP class is the exponent $\beta \simeq 0.25$ instead of $\beta_{D P} \simeq 0.276$. However, the data points in Fig. 8 in that paper would also easily match well with a power-law scaling with the DP exponent within the range of the error bars. A decade later, Bottin et al. [39, 58] conducted the first series of experiments in PCF aiming to clarify the continuous nature of the transition to sustained turbulence. Based on the seemingly-discontinuous curve of the mean turbulent fraction and the qualitative comparison between experiments and models, they claimed that the transition is discontinuous, i.e., of the first order. Since the system in their study was small and contained only few spots, the results might be influenced by the finite-size effects and thus the conclusion remains controversial. Although recent numerical studies in PCF [43, 59] appears to support a discontinuous nature of the transition, the viewpoint was challenged by both the numerical and experimental observations in pipe flow [12, 60]. To resolve this mystery, quantitative evidence (e.g. critical exponents) are needed. Unfortunately in pipe flow the extremely large time scale $\left(\sim 10^{7-8}\right)$ of dynamical events (turbulence decay or spreading) at criticality makes the measurement of critical exponents beyond the current capabilities. As we will show later in this thesis, the time scale in PCF is several orders of magnitude smaller than that in a pipe, which thus provides a unique opportunity to measure the critical exponents at criticality.

\subsection{Stability of rotating flows in astrophysical accretion disks}

Relevant physical mechanisms for the onset of turbulence in linearly stable flows like PCF and pipe flow are well established. Due to the non-normality of the linearized system, initial disturbances are algebraically amplified by a large factor before the viscosity of the fluid damps out all the fluctuations. This effect is known as transient growth 61 63. Under sufficiently large initial perturbations, linear transient growth will, by pumping energy from the laminar flow to big structures such as streamwise streaks and vorticities, distort strongly the laminar flow. This distortion makes nonlinear interactions between modes no longer negligible, which eventually causes the flow break down to turbulence. The relevance of such mechanisms is much less understood for other shear flows. One interesting example is the turbulence in cold or 
weakly-ionized astrophysical accretion disks, where rotation plays a key role besides shear.

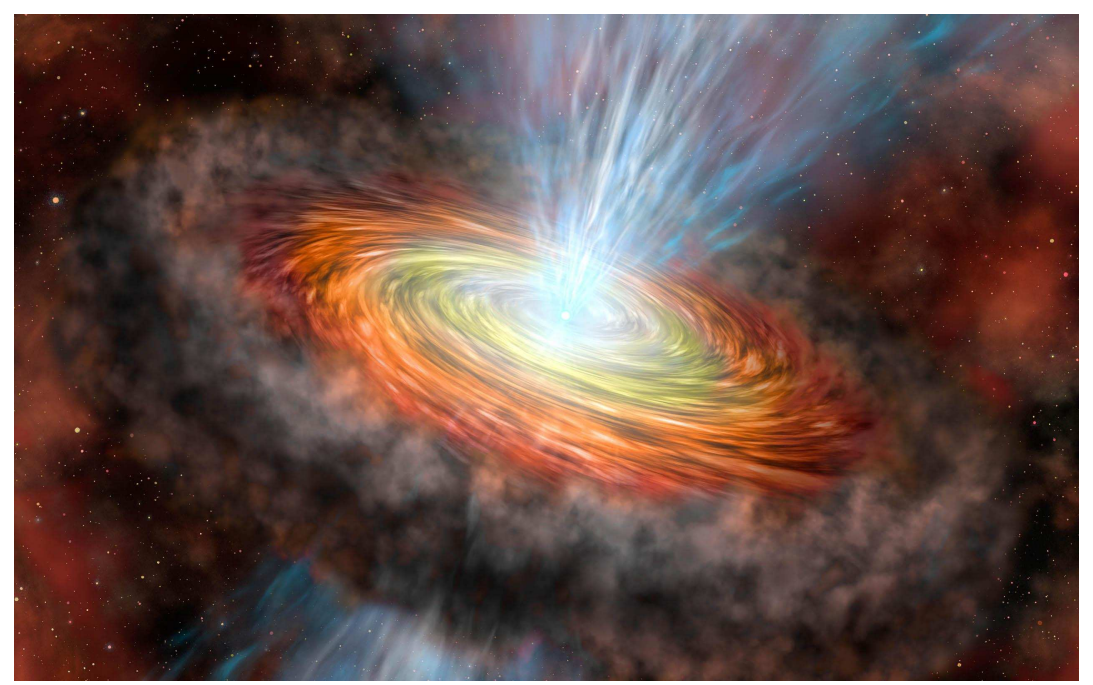

FigURE 1.5: Artist rendering of astrophysical accretion disk: turbulent motions of fluids accompany with the outbursts of radiation jets. (Credit: Gemini Observatory)

As an ubiquitous phenomenon in the universe, astrophysical accretion disks are thinlayer rotating flows of matter (dust, gas, ions, etc) around a massive central body, such as a star or a black hole (see Fig. 1.5). In Keplerian flows, the angular velocity in the disk is $\Omega^{K}(r) \sim r^{-3 / 2}$, which leads to an increasing angular momentum outwards. As matter spirals towards the central body under gravity, it incurs a loss of gravitational energy and angular momentum. The gravitational energy is tranferred into heat or radiated by the emission of electromagnetic radiation. On the other hand, the loss of angular momentum due to the mass falling into the center must be compensated by an angular momentum gain of the mass far from the center, in order that the total angular momentum is conserved in the whole disk. In rotating flows, it must be the torque induced by the azimuthal shear stresses between different layers that causes the redistribution of angular momentum in the disk. Since molecular viscosity is known to be too small to match the observed accretion rates, turbulent viscosity, which can be several orders of magnitude larger than the molecular one, was proposed by Shakura and Sunyaev [64] to account for disk accretion. Observational evidences of turbulent motion have been reported in some disks [65 67]. A more intriguing question is how turbulence arises, given that the Keplerian velocity profile is linearly stable according to Rayleigh criterion [2]. In hot or ionized disks, magnetic fields in stars act on the ionized rotating fluids, which causes linear instability if the fluid velocity decreases outwards [68 70]. This is nowadays called "magnetorotational instability" (MRI). However, in cold disks a clear candidate is still lacking to explain the origin of turbulence. As shown in pipe and Couette flows, nonlinear mechanisms can give rise to sustained turbulence, despite the linear stability of the laminar profiles. Turbulence 
was also observed in case of stationary inner cylinder [4] as well as in the counterrotating regimes [8] in TCF, which are also linearly stable.

For decades, TCF has been employed to probe subcritical turbulence in accretion disks 71 75], as initially proposed by Zeldovich [76]. The main reasons are as follows. Firstly, laminar TCF can realize the outwards increasing distribution of angular momentum as encountered in Keplerian flows and it can approach asymptotically the Keplerian velocity profile. On the other hand, physical processes involving in disk accretion are very complicated, including hydrodynamics, magnetic fields, radiation, thermal stratification, etc. The irregular geometry and free-surface boundaries of the disks adds more complexity to the real physical problem. Because of these difficulties in real disks, TCF is therefore an appropriate laboratory framework to understand as a first step the pure hydrodynamics in the disks. A more detailed comparison between accretion disks and TCF can be found in [77].

Recently, conflicting results on whether subcritical hydrodynamic turbulence exists in the quasi-Keplerian regime in TCF (regime II in Fig. 1.2) have been reported. All cited experiments do not include magnetic or other physical fields except the hydrodynamic flows. Ji et al. [72, 73] measured the Reynolds stress or the $\beta$ parameter introduced by Richard and Zahn [71] at discrete interior locations at Re up to $2 \times 10^{6}$ and found that experimentally measured $\beta$ is far below the value calculated from astrophysical observations and hence cannot explain the observed transportation rate of angular momentum in disks. However, this was challenged by the results from another two groups [74], where strong level of angular momentum transport was observed in independent experiments in TCF at similar Re as in [72]. The estimated $\beta$ based on the torque measurement in the inner cylinder is at similar level as in astrophysical disks. However, differences between the experiments, such as geometry (axial-length-to-gap aspect ratio $\Gamma$ ) and end-cap treatment as well as the measured physical quantities, make a direct comparison very difficult [78]. In TCF experiments, the finite-size effect due to the presence of end caps is unavoidable and not expected in astrophysical disk flows. This experimentally imposed axial boundary condition drives the nearby fluids at the same rotational speed as the end caps and causes instabilities penetrating into the bulk flow, which is known as the Ekman effect. The team of Ji claimed that, although they have a very small aspect ratio $\Gamma=2.104$, the split end caps in their setup [72] help to mitigate and minimize the Ekman effects, whereas Paoletti et al. have longer cylinders $(\Gamma=11.47)$ and argued that the measurement in the middle section of the inner cylinder would be free of Ekman effects. By contrast, direct numerical simulations of above experimental flows showed that the presence of top and bottom end caps leads to strong deviations from the Keplerian velocity profile and that these deviations cause turbulence to arise [75]. It turns out that experiments alone are hard 
to fully address the subtle question whether disks can be turbulent for purely hydrodynamic reasons. This motivated us to perform direct numerical simulations of TCF with axially periodic cylinders and hence free of end-cap effects.

\subsection{Thesis outline}

The thesis addresses the following four questions.

1. What is the critical Reynolds number in PCF beyond which turbulence is sustained?

2. Is this transition a continuous or discontinuous phase transition in statistical physics?

3. Can sustained turbulence in quasi-Keplerian TCF arise for purely hydrodynamic mechanisms?

4. How does the Earth rotation influence linearly stable rotating flow?

For the questions $1-3$, we solve numerically the governing equations of fluid flows and conduct the direct numerical simulations (DNS) of TCF. Simulations concerning question 1-2 are performed in regime I of the parameter space shown in Fig. 1.2, where the flow approaches asymptotically the PCF limit as stated in Sec. 1.2, The Reynolds numbers of interest here are at low range, $R e \sim \mathcal{O}\left(10^{2}\right)$. Due to the stochastic nature of turbulence in $\mathrm{PCF}$, large ensemble of simulations are performed and statistical methods are employed to analyze the data. Regime II in Fig. 1.2 is often referred to as the quasi-Keplerian regime, which is related to Question 3. To reach the Reynolds number of astrophysical relevance, a highly efficient and parallelized DNS code has been developed. The code will be presented in Chapter 2 and we perform simulations at shear Re up to $1 \times 10^{5}$. To understand the influence of Earth rotation on linearly stable flows, we choose the simplest shear flow PCF and put it under system rotation. We solve the linearized governing equations, conduct the linear stability analysis and transient growth study.

The structure of the rest of the thesis is as follows. The governing equations of fluid flows and the numerical method are presented in Chapter 2, which also provides a detailed demonstration of the high efficient parallel code nsCouette. In Chapter 3, the results on the onset of sustained turbulence in PCF are shown. By balancing the time scale of the decay and spreading dynamical processes, a distinct Re is obtained, $R e \simeq 325$, which defines a lower bound for the real critical point $R e^{c}$ of the transition. At criticality, the critical exponents are measured and the subcritical transition 
to sustained turbulence is found to be a continuous phase transition in spatially extended systems, which may fall into the DP universality class. Chapter 4 presents simulations in the quasi-Keplerian regime of TCF. Starting with optimal perturbations, our simulations reveal a secondary instability in the axial direction. Through nonlinear interactions this instability gives rise to transient turbulence. The influence of Earth rotation on shear flow experiments is shown in Chapter 5. It is found that infinitesimal system rotation perpendicular to the walls of PCF causes the system linear instability. The results of the thesis are summarized and discussed in Chapter 6. 

"THERE ARE 3 RULES TO FOLLOW WHEN PARALLELIZING LARGE CODES. UNFORTUNATELY, NO ONE KNOWS WHAT THESE RULES ARE."

\section{A COMPUTER SCIENTIST}

"ANYONE WHO CONSIDERS ARITHMETICAL METHODS of PRODUCING RANDOM Digits IS, OF COURSE, IN A STATE OF SIN."

John Von Neumann (1903 - 1957)

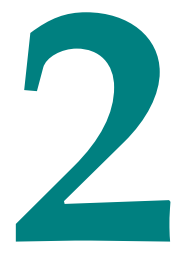

\section{A HIGHLY EFFICIENT PARALLEL DNS CODE nsCouette 1}

\section{$2.1 \quad$ Introduction}

Starting with the study of homogeneous isotropic turbulence conducted by Orszag and Patterson [79], direct numerical simulation (DNS) has been proven as a very powerful approach to explore the physics of turbulent flows (see Ref. [41], [42]). It has been widely used in fundamental research on both transitional and fully-developed turbulence in boundary layers over a flat plate (e.g. 80], [81]), channel (e.g. [82], [83]), pipe (e.g. [84], [85]) and Couette flows (e.g. [86], [87]). Distinguished from Reynoldsaveraged equations (RANS) and Large-Eddy simulation (LES), a carefully performed DNS resolves all temporal and spatial scales relevant to turbulence and thus provides data of high fidelity. Its advantage is also its main drawback: resolving the physics of turbulence implies a scaling of the computational complexity as $\mathcal{O}\left(R e^{3}\right)$ [88].

In this section we present a highly efficient DNS code for TCF with axially periodic boundary conditions using a hybrid two-level parallelization strategy. It enables DNS to be performed up to $R e \sim \mathcal{O}\left(10^{5}\right)$, and thus provides access to a broad range in

\footnotetext{
${ }^{1}$ The content in this Chapter is mainly from the paper: L. Shi, M. Rampp, B. Hof, M. Avila, A Hybrid MPI-OpenMP Parallel Implementation for Pseudospectral Simulations: Application to TaylorCouette Flow, accepted with minor revision to Computers Ef Fluids, (2014).
} 
the parameter space of TCF, including quasi-Keplerian flows at experimentally relevant Reynolds numbers. The most efficient method for discretizing partial differential equations with periodic boundary conditions is the spectral Fourier-Galerkin method, so we use this in the axial and azimuthal directions. Many authors use the spectral Galerkin-method in the non-periodic radial direction by employing Chebyshev, Legendre or Jacobi polynomials. The two latter render however a computational complexity of $\mathcal{O}\left(M^{2}\right)$, where $M$ is the degree of the approximation, due to the lack of fast transformations between physical and spectral spaces. This makes computations too expensive at large Reynolds number. In contrast, with the Chebyshev method the fast cosine transform allows it to keep the cost at $\mathcal{O}(M \log (M))$. However, in order to use accurate quadratures the projection basis must be different from the basis used to discretize the Navier-Stokes equations (Petrov-Galerkin method) [89, 90]. On the other hand, if the spectral method is used directly at a collocation grid (in physical space) the resulting differentiation matrices are dense. Hence the solution of the Poisson equations, for example with the diagonalization method [1], requires $\mathcal{O}\left(M^{2}\right)$ operations. A common drawback of all the aforementioned spectral methods is that the density of collocation nodes towards the boundaries scales as $\mathcal{O}\left(M^{2}\right)$. Although this allows to properly resolve boundary layers with relatively low resolutions, at large Reynolds numbers the clustering is excessive and the required resolution is often given by the spacing of nodes far from the boundaries. Moreover, this clustering poses a severe restriction on the time step of $\Delta t=\mathcal{O}\left(M^{-2}\right)$ because of the CFL condition. Although transformations of the node distribution have been proposed [92], these result in the loss of the spectral convergence. Finally, it becomes impractical to use the Chebyshev method for large resolutions $M \gtrsim 600$, as needed in the simulation of turbulence at large $R e$ (see $\$ 2.5$ ). For these reasons we use the high-order finite-difference (FD) method in the radial direction, which makes the stretching of grid nodes straightforward.

The dimensionless, incompressible Navier-Stokes equations in primitive variables are integrated in time with a second-order $\mathcal{O}\left(\Delta t^{2}\right)$ time-splitting scheme proposed by Hugues \& Randriamampianina [93]. The scheme is semi-implicit and is second-order accurate also for the pressure, rendering a very small $\mathcal{O}\left(\Delta t^{3}\right)$ slip-velocity error at the boundary while fulfilling the incompressibility constraint. It is straightforward to implement: it avoids staggered grids and requires the solution of five equations of Poisson or Helmholtz type. The nonlinear advective term is computed in physical space with the pseudospectral method. The code is parallelized by combining the Message Passing Interface (MPI) and the Open Multiprocessing (OpenMP) paradigms. The Fourier-Galerkin method leads to mode-decoupled linear equations, which makes the one-dimensional MPI parallelization rather straightforward to implement. OpenMP threading within MPI tasks allows to efficiently use modern high performance computing (HPC) architectures and mitigates the overhead induced by MPI All-to-all inter-task communications which are typical of spectral methods. 


\subsection{Navier-Stokes equations}

The equations governing the motion of an incompressible fluid of kinematic viscosity $\nu$ and constant density $\rho$ is called Navier-Stokes equations. Its mathematical form consists of a equation of momentum and a equation of mass conservation,

$$
\partial_{t} \mathbf{u}+\mathbf{u} \cdot \nabla \mathbf{u}=-\frac{1}{\rho} \nabla p^{h}+\nu \Delta \mathbf{u}, \quad \nabla \cdot \mathbf{u}=0
$$

where $\mathbf{u}(\mathbf{r}, t)$ is the velocity field and $p^{h}(\mathbf{r}, t)$ is the hydrodynamic pressure. Here cylindrical coordinates $\mathbf{r}=(r, \theta, z)$ are used. The geometry and parameters of the TCF system are shown in Fig. 1.2 and presented in $\$ 1.2$. At the cylinders no-slip boundary conditions are applied, whereas in the axial direction periodic boundary conditions are imposed to avoid end-wall effects. This approximates the case of very long cylinders. In the azimuthal direction periodic boundary conditions occur naturally. However, it is often computationally convenient to simulate only an angular section $L_{\theta} \leq 2 \pi$ of the cylinders, and periodic boundary conditions are then used for $\theta \in\left[0, L_{\theta}\right]$. This is justified provided that the correlation length of the turbulent flow in the azimuthal direction is shorter than $r_{i} L_{\theta}$ [87].

Henceforth, all variables will be rendered dimensionless using $d, \tau=d^{2} / \nu$, and $\nu^{2} / d^{2}$ as units for space, time, and the reduced pressure $p=p^{h} / \rho$, respectively. The NavierStokes equations (2.1) for this scaling become

$$
\begin{aligned}
\partial_{t} \mathbf{u}+\mathbf{u} \cdot \nabla \mathbf{u} & =-\nabla p+\Delta \mathbf{u} \\
\nabla \cdot \mathbf{u} & =0 .
\end{aligned}
$$

In cylindrical coordinates the equations read

$$
\begin{aligned}
\left(\partial_{t}+\mathbf{u} \cdot \nabla\right) u_{r}-u_{\theta}^{2} / r & =-\partial_{r} p+\Delta u_{r}-u_{r} / r^{2}-2 \partial_{\theta} u_{\theta} / r^{2} \\
\left(\partial_{t}+\mathbf{u} \cdot \nabla\right) u_{\theta}+u_{\theta} u_{r} / r & =-\partial_{\theta} p / r+\Delta u_{\theta}-u_{\theta} / r^{2}+2 \partial_{\theta} u_{r} / r^{2} \\
\left(\partial_{t}+\mathbf{u} \cdot \nabla\right) u_{z} & =-\partial_{z} p+\Delta u_{z}, \\
u_{r} / r+\partial_{r} u_{r}+\partial_{\theta} u_{\theta} / r+\partial_{z} u_{z} & =0 .
\end{aligned}
$$

with $\nabla=\left(\partial_{r}, \partial_{\theta} / r, \partial_{z}\right)$ and $\Delta=\partial_{r} / r+\partial_{r r}^{2}+\partial_{\theta \theta}^{2} / r^{2}+\partial_{z z}^{2}$. Note that the Reynolds numbers enter the system through the boundary conditions

$$
\begin{aligned}
u_{\theta}\left(r_{i, o}, \theta, z\right) & =\operatorname{Re}_{i, o}, \\
u_{r, z}\left(r_{i, o}, \theta, z\right) & =0, \\
\mathbf{u}(r, \theta, z) & =\mathbf{u}\left(r, \theta+L_{\theta}, z\right), \\
\mathbf{u}(r, \theta, z) & =\mathbf{u}(r, \theta, z+\Gamma) .
\end{aligned}
$$


By taking the divergence of the first equation and then applying the incompressibility condition, we obtain a Poisson equation for the pressure,

$$
\Delta p=-\nabla \cdot \mathbf{N}(\mathbf{u}), \quad \text { where } \quad \mathbf{N}(\mathbf{u})=\mathbf{u} \cdot \nabla \mathbf{u},
$$

with consistent boundary conditions 94

$$
\left.\partial_{n} p\right|_{r=r_{i, o}}=\mathbf{n} \cdot\left[-\partial_{t} \mathbf{u}-\mathbf{N}(\mathbf{u})+\Delta \mathbf{u}\right] .
$$

As explained in 92.3 .2 , this equation will be solved for the pressure prediction.

\section{$2.3 \quad$ Numerical method}

The governing equations (2.3) are solved for the primitive variables $(\mathbf{u}, p)$. We discretize the equations with a combination of the spectral-Fourier method with the finite-difference method (FD) in space, whereas time is advanced with a semi-implicit fractional-step method proposed by Hugues and Randriamampianina [93], who employ a second-order-accurate backward-differentiation formula with second-order extrapolation for the nonlinear term. The pseudospectral technique with 3/2-dealiasing [79] is applied to compute the nonlinear term $\mathbf{N}(\mathbf{u})$ in physical space.

\subsubsection{Spatial discretization}

In the periodic axial and azimuthal directions, the velocity field and pressure are approximated as

$$
\begin{aligned}
\mathbf{u}(r, \theta, z) & =\sum_{l=-L}^{L} \sum_{n=-N}^{N} \hat{\mathbf{u}}^{l n}(r) e^{i\left(l k_{z} z+n k_{\theta} \theta\right)}, \\
p(r, \theta, z) & =\sum_{l=-L}^{L} \sum_{n=-N}^{N} \hat{p}^{l n}(r) e^{i\left(l k_{z} z+n k_{\theta} \theta\right)},
\end{aligned}
$$

where $k_{z}$ is the minimum (fundamental) axial wavenumber and fixes the axial nondimensional length $\Gamma=2 \pi / k_{z}$ of the computational domain. Similarly, $L_{\theta}=2 \pi / k_{\theta}$ is the azimuthal arc degree; $k_{\theta}=1$ corresponds to the natural periodic boundary condition in the azimuthal direction, whereas $k_{\theta}=4$ corresponds to one quarter of an annulus. The hat symbol ${ }^{\wedge}$ in (2.7) denotes quantities in Fourier space and the tuple $(L, N)$ determines the spectral numerical resolution.

By substituting (2.7) into (2.3) and projecting the result onto a basis $e^{-i\left(l k_{z} z+n k_{\theta} \theta\right)}(l=$ $-L, \cdots, L ; n=-N, \cdots, N)$, we obtain the mode-decoupled Navier-Stokes equations. 
For each Fourier mode $(l, n)$, they read

$$
\begin{aligned}
& \partial_{t} \hat{u}_{r}+\hat{N}_{r}=-\partial_{r} \hat{p}+\hat{\Delta} \hat{u}_{r}-\hat{u}_{r} / r^{2}-2 i n k_{\theta} \hat{u}_{\theta} / r^{2}, \\
& \partial_{t} \hat{u}_{\theta}+\hat{N}_{\theta}=-i n k_{\theta} \hat{p} / r+\hat{\Delta} \hat{u}_{\theta}-\hat{u}_{\theta} / r^{2}-2 i n k_{\theta} \hat{u}_{r} / r^{2}, \\
& \partial_{t} \hat{u}_{z}+\hat{N}_{z}=-i l k_{z} \hat{p}+\hat{\Delta} \hat{u}_{z} .
\end{aligned}
$$

Here $\hat{\Delta}=\partial_{r} / r+\partial_{r r}-n^{2} k_{\theta}^{2} / r^{2}-l^{2} k_{z}^{2}$ and the superscripts $(l, n)$ have been omitted for clarity. Note that the nonlinear term couples Fourier modes and it is thus computed in physical space with the pseudospectral method. Details of the implementation and parallelization of the nonlinear term are given in \$2.4. Equations (2.8) couple the radial and azimuthal velocities. By applying the following change of variables [95]

$$
\begin{aligned}
& \hat{u}_{+}=\hat{u}_{r}+i \hat{u}_{\theta}, \\
& \hat{u}_{-}=\hat{u}_{r}-i \hat{u}_{\theta},
\end{aligned}
$$

to equation (2.8), we yield the decoupled equations

$$
\begin{aligned}
\partial_{t} \hat{u}_{+}(r)+\hat{N}_{+}(r) & =-\partial_{r} \hat{p}(r)+n k_{\theta} \hat{p}(r) / r+\left(\hat{\Delta}-1 / r^{2}-2 n k_{\theta} / r^{2}\right) \hat{u}_{+}, \\
\partial_{t} \hat{u}_{-}(r)+\hat{N}_{-}(r) & =-\partial_{r} \hat{p}(r)-n k_{\theta} \hat{p}(r) / r+\left(\hat{\Delta}-1 / r^{2}+2 n k_{\theta} / r^{2}\right) \hat{u}_{-}, \\
\partial_{t} \hat{u}_{z}(r)+\hat{N}_{z}(r) & =-i l k_{z} \hat{p}(r)+\hat{\Delta} \hat{u}_{z},
\end{aligned}
$$

where $\hat{N}_{ \pm}=\hat{N}_{r} \pm i \hat{N}_{\theta}$.

We use a standard high-order, central finite-difference method to approximate the radial derivatives in equations (2.9) (see Ref. [96]). The radial nodes are distributed as 92 ]

$$
r_{j}=\frac{1+\eta}{2(1-\eta)}+\frac{\sin ^{-1}(-\alpha \cos (\pi j / M))}{2 \sin ^{-1} \alpha}, \quad j=0, \ldots, M .
$$

For $\alpha=1$ the grid is uniform, whereas for $\alpha \rightarrow 0$ the Chebyshev collocation points are obtained. Here stencils of $n_{s}=9$ points, corresponding to a scheme of formally order 7 was found to give the best compromise in our tests. Note that we reduce the stencil length gradually towards the boundaries in order to keep the FD-matrices banded. We show in $\$ 2.5$ that due to the clustering of nodes near the walls with typical values of $\alpha=0.5$ this reduction of the order of accuracy does not produce a larger error at the boundaries.

With $(L, N)$ Fourier modes and $M$ radial nodes, the number of grid points in physical space is $\left(n_{r}, n_{\theta}, n_{z}\right)=(M, 2 N+1,2 L+1)$ in radial, azimuthal and axial direction, respectively. Note that we dealise the nonlinear term by computing it in a grid of $(M, 3 N+1,3 L+1)$ points. 
Chapter 2. A highly efficient parallel DNS code nsCouette

\subsubsection{Temporal scheme}

A stiffly stable temporal scheme based on a backward differentiation formula with extrapolation for the nonlinear term is adopted (see Ref. [93, 97]). It reads

$$
\frac{3 \mathbf{u}^{i+1}-4 \mathbf{u}^{i}+\mathbf{u}^{i-1}}{2 \Delta t}+2 \mathbf{N}^{i}(\mathbf{u})-\mathbf{N}^{i-1}(\mathbf{u})=-\nabla p^{i+1}+\Delta \mathbf{u}^{i+1} .
$$

This is often referred in the literature to as Adams-Bashforth backward-difference method of second order (AB2BD2). The viscous terms are discretized implicitly, whereas the nonlinear terms are treated explicitly. At each time step, equation (2.11) is solved through a fractional step method proposed by Hugues and Randriamampianina [93]. The method is summarized below. Here $\left(\hat{\mathbf{u}}^{i}, \hat{p}^{i}\right)$ denote the spectral coefficients at the $i^{\text {th }}$ time step.

1. Obtain spectral coefficients of the nonlinear term, $\hat{\mathbf{N}}^{i}(\mathbf{u})$, using the $3 / 2$-dealiasing rule

- Do matrix-vector multiplication to calculate $\partial_{r} \hat{\mathbf{u}}^{i}$ (FD method)

- Compute dot product in Fourier space to calculate $\partial_{\theta} \hat{\mathbf{u}}^{i}$ and $\partial_{z} \hat{\mathbf{u}}^{i}$

- Perform Fourier transform of $\partial_{r, \theta, z} \hat{\mathbf{u}}^{i}$ and $\hat{\mathbf{u}}^{i}$ to obtain the velocity field and all its derivatives in physical space;

- Calculate $\mathbf{N}^{i}(\mathbf{u})=\mathbf{u}^{i} \cdot \nabla \mathbf{u}^{i}$;

- Perform inverse Fourier transform to obtain the spectral coefficients $\hat{\mathbf{N}}^{i}(\mathbf{u})$.

2. Obtain the pressure prediction, $\hat{p}^{*}$ : solve the Poisson equation

$$
\Delta \hat{p}^{*}=\nabla \cdot\left[-2 \hat{\mathbf{N}}^{i}(\mathbf{u})+\hat{\mathbf{N}}^{i-1}(\mathbf{u})\right],
$$

with consistent Neumann boundary conditions (2.6). In practice, to remove the singularity of the problem, the Neumann boundary condition at the outer cyliner is replaced by the Dirichlet boundary condition.

3. Obtain the velocity prediction, $\hat{\mathbf{u}}^{*}$ : solve the three Helmholtz equations

$$
\frac{3 \hat{\mathbf{u}}^{*}-4 \hat{\mathbf{u}}^{i}+\hat{\mathbf{u}}^{i-1}}{2 \Delta t}+2 \hat{\mathbf{N}}^{i}(\mathbf{u})-\hat{\mathbf{N}}^{i-1}(\mathbf{u})=-\nabla \hat{p}^{*}+\Delta \hat{\mathbf{u}}^{*}
$$

with Dirichlet boundary conditions (2.4).

4. Correct via an intermediate variable $\phi=\frac{2 \Delta t\left(\hat{p}^{i+1}-\hat{p}^{*}\right)}{3}$ : The incompressibility condition $\nabla \cdot \hat{\mathbf{u}}^{i+1}=0$ leads to a Poisson equation for $\phi$ with homogeneous 
Neumann boundary conditions (see Ref. [93, 94])

$$
\begin{gathered}
\Delta \phi=\nabla \cdot \hat{\mathbf{u}}^{*}, \\
\left.\partial_{r} \phi\right|_{r=r_{i, o}}=0
\end{gathered}
$$

5. Compute pressure and velocity correction, $\hat{p}^{i+1}$ and $\hat{\mathbf{u}}^{i+1}$ :

$$
\begin{aligned}
\hat{p}^{i+1} & =\hat{p}^{*}+3 \phi /(2 \Delta t) \\
\hat{\mathbf{u}}^{n+1} & =\hat{\mathbf{u}}^{*}-\nabla \phi
\end{aligned}
$$

6. Go back to step 1

The Navier-Stokes equations are thus advanced in time by solving five systems of linear equations (2.12)-(2.14), of Poisson or Helmholtz type. This method accounts for a divergence-free velocity field and a small slip at the wall of the order of $\mathcal{O}\left(\Delta t^{3}\right)$ in the tangential velocities, $u_{z}$ and $u_{\theta}$. We note that the method was originally developed and tested [93] for the two-dimensional Navier-Stokes equation discretized on a ChebyshevChebyshev collocation grid, and the Poisson and Helmholtz equations were solved using the double diagonalization method. Here, the FD-discretized Poisson and Helmholtz render banded matrices which are solved with the LU-method. The decompositions are precomputed at the beginning of a simulation and at each time step only backward and forward substitutions need to be computed, resulting in an operation count of $\mathcal{O}(M)$ for the solution of each system. Note that for the axially and azimuthally invariant Fourier mode, $n=l=0$, the Poisson equations (2.12) and (2.14) are singular: their solution is defined up to a constant because of the Neumann boundary conditions. Here a Dirichlet homogeneous boundary condition was employed at the outer cylinder to select a particular solution.

\subsection{Parallelization scheme and its implementation}

A hybrid MPI-OpenMP parallelization strategy is adopted for the implementation of the code. Since the linear equations (2.12)-(2.14) are mode-independent, it is convenient to employ an MPI-based, one-dimensional domain decomposition (also known as "slab" decomposition, Fig. 2.1): The Fourier coefficients $\left(\hat{u}_{+}, \hat{u}_{-}, \hat{u}_{z}, \hat{p}\right)$ corresponding to different modes are distributed across the MPI tasks which allows to solve equations (2.12)-(2.14) concurrently, without any inter-task communication. Each of the $N_{\text {tasks }}$ MPI tasks operates on data corresponding to a number of $m_{\theta} \cdot m_{z} / N_{\text {tasks }}$ modes, where $\left(m_{r}, m_{\theta}, m_{z}\right)=(M, N+1,2 L)$ are the dimensions of variables in Fourier space. OpenMP threading inside each MPI task allows to efficiently exploit the remaining coarse-grained parallelism (see below). 
Chapter 2. A highly efficient parallel DNS code nsCouette
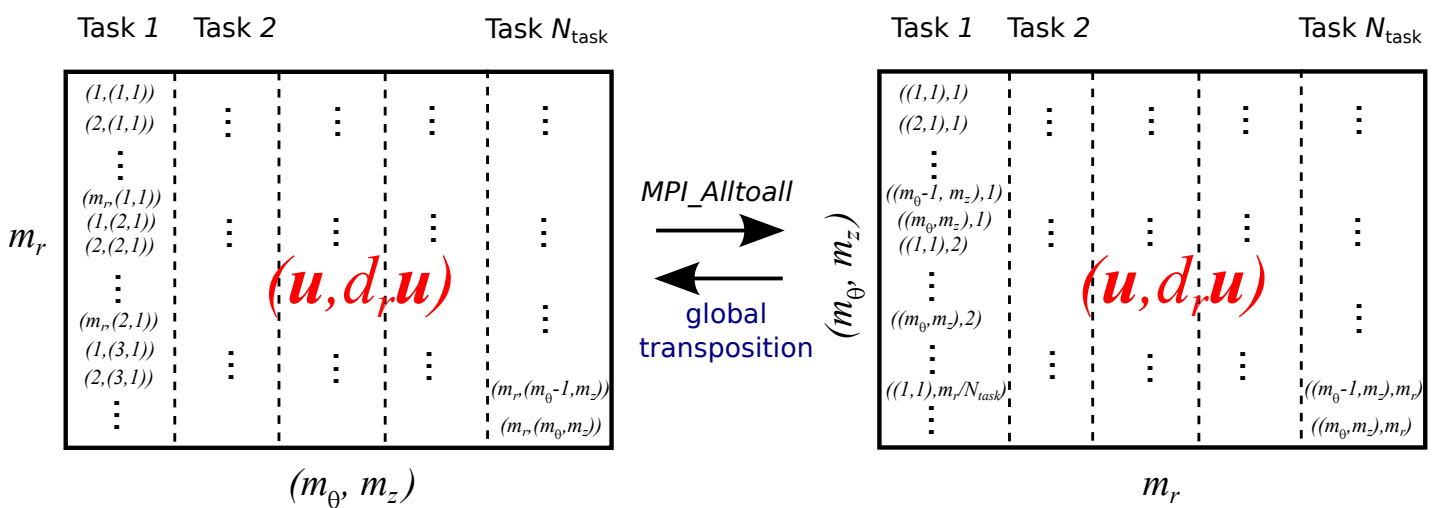

Figure 2.1: Schematic of the MPI-based, one-dimensional "slab" domain decomposition and the global transposition by using the function MPI_Alltoall(). Modeindependent spectral coefficients in Fourier space are distributed among different MPI tasks. Each variable has a dimension of $\left(m_{r}, m_{\theta}, m_{z}\right)$.

We compute the nonlinear term (step 1 in Section 2.3.2) by performing global matrix transpositions (Fig. 2.1) of the discretized fields $\partial_{r} \hat{\mathbf{u}}$ and $\hat{\mathbf{u}}$ such that for each radial point the complete spectrum of Fourier modes is localized in one MPI task. This requires a collective communication operation of type "all-to-all" but allows to most efficiently compute the Fourier transformations and the derivatives with respect to the spectral coordinates, namely $\theta$ and $z$. Finally, inverse transpositions are performed for the resulting array $\hat{\mathbf{N}}$.

In our applications, typically $m_{\theta} \cdot m_{z} \gg m_{r}$ applies, i.e. there are much more Fourier modes than radial grid points. Hence, the number of MPI tasks in our slab decomposition is bounded by $N_{\text {tasks }} \leq m_{r}$ (cf. Fig.. 2.1) and consequently the maximum achievable parallel speedup would be limited by $m_{r}$. However, OpenMP threads allow to parallelize over the $m_{\theta} \cdot m_{z} / N_{\text {tasks }}$ modes within a MPI task while retaining the one-dimensional MPI domain decomposition which is conceptually straightforward to implement. Similarly, we can exploit concurrency in the nonlinear part if $N_{\text {tasks }}<m_{r}$ applies. In addition, the Fourier transformations and the individual partial derivatives required for evaluating $\mathbf{u} \cdot \nabla \mathbf{u}$ are computed concurrently and the transposition of $\partial_{r} \hat{\mathbf{u}}$ is overlapped with the computation of $\mathbf{u}, \partial_{\theta} \mathbf{u}$, and $\partial_{z} \mathbf{u}$.

Theoretically, this strategy allows to utilize a number of $\min \left(m_{r}, m_{\theta} \cdot m_{z}\right) \cdot N_{\text {threads }}$ processor cores where $N_{\text {threads }}$ is the maximum number of threads a shared-memory compute node provides. Current high performance computing (HPC) platforms feature at least 16 cores with up to 32 logical threads per node (e.g. Intel Sandy-Bridge E5 clusters), and thread-based concurrency on the node-level is expected to increase substantially in the near future, in particular with the upcoming many-core processors and GPU-accelerated nodes [98]. In practice, we achieve excellent parallel efficiencies when MPI tasks are mapped to the individual "sockets" (i.e. CPUs or NUMA domains) of a compute node and the number of OpenMP threads equals the number of physical 
cores per socket. Due to the smaller number of MPI tasks per node (compared with a plain MPI parallelization) the amount of inter-node communications is reduced in the global transposition. This transposition, which is implemented by MPI_Alltoall collective communication and task-local transpositions, ultimately limits the overall parallel scalability of the code at high task counts (see Section 2.6).

The code is implemented in FORTRAN 90 and has been ported to a number of major HPC architectures, including IBM Power and BlueGene, as well as compute clusters based on x86_64 processors and high-performance interconnects such as InfiniBand. We employ vendor-optimized BLAS and LAPACK routines for the matrix-vector multiplication (BLAS level-2 routine DGEMV) and the linear solvers (variants of LAPACK routines DGBTRF, DGBTRS taken e.g. from the Intel Math Kernel Library, or IBM ESSL), respectively, and utilize the FFTW library [99] for performing the Fourier transformations in the nonlinear part of the code. For data output we employ the parallel HDF5 libraries which enable collective output of the MPI-distributed data into a single file in a transparent and efficient way. This facilitates data handling, post-processing and visualization, e.g. with VisIT or Paraview (cf. Fig. 2.9).

\subsection{Numerical Accuracy and Code Validation}

The code has been tested over a wide range of Reynolds numbers $R e \in[50,100000]$. A number of specific test cases will be given in the following.

\subsubsection{Laminar flow}

We firstly computed the laminar velocity profile, which is also known as circular Couette flow. It can be expressed as $\mathbf{U}=\left(0, U_{\theta}(r), 0\right)$, where $U_{\theta}(r)=C_{1} r+C_{2} / r$ with $C_{1}=\left(R e_{o}-\eta R e_{i}\right) /(1+\eta)$ and $C_{2}=\eta\left(R e_{i}-\eta R e_{o}\right) /\left((1-\eta)\left(1-\eta^{2}\right)\right)$, corresponding to pure rotary shear flow . The tests were performed at $R e_{i}=50, R e_{o}=200$ and at $\eta=0.5$. A non-uniform grid according to formula (2.10) is used in the radial directio n, clustering at the boundaries. Fig. 2.2(top) shows the numerical velocity and pressure profiles for $\alpha \rightarrow 0$ (Chebyshev points ) and $n_{r}=32$, which match well with the theoretical curves (dashed lines). The distributions of the relative error $\epsilon_{u}(r)=\left|\frac{u_{\theta}-U_{\theta}}{U_{\theta}}\right|$ along the radial direction are shown in Fig. 2.2(bottom) for different stencil lengths $n_{s}$. In FD method, the stencil length is the number of consecutive points used to approximate the derivatives. The error is quite smoothly dist ributed at the bulk flow. As $n_{s}$ is increased, the relative error decreases until approaching the machine precision. In order to make the best compromise be tween the computing time and the accuracy, $n_{s}=9$ is chosen for the following tests. 

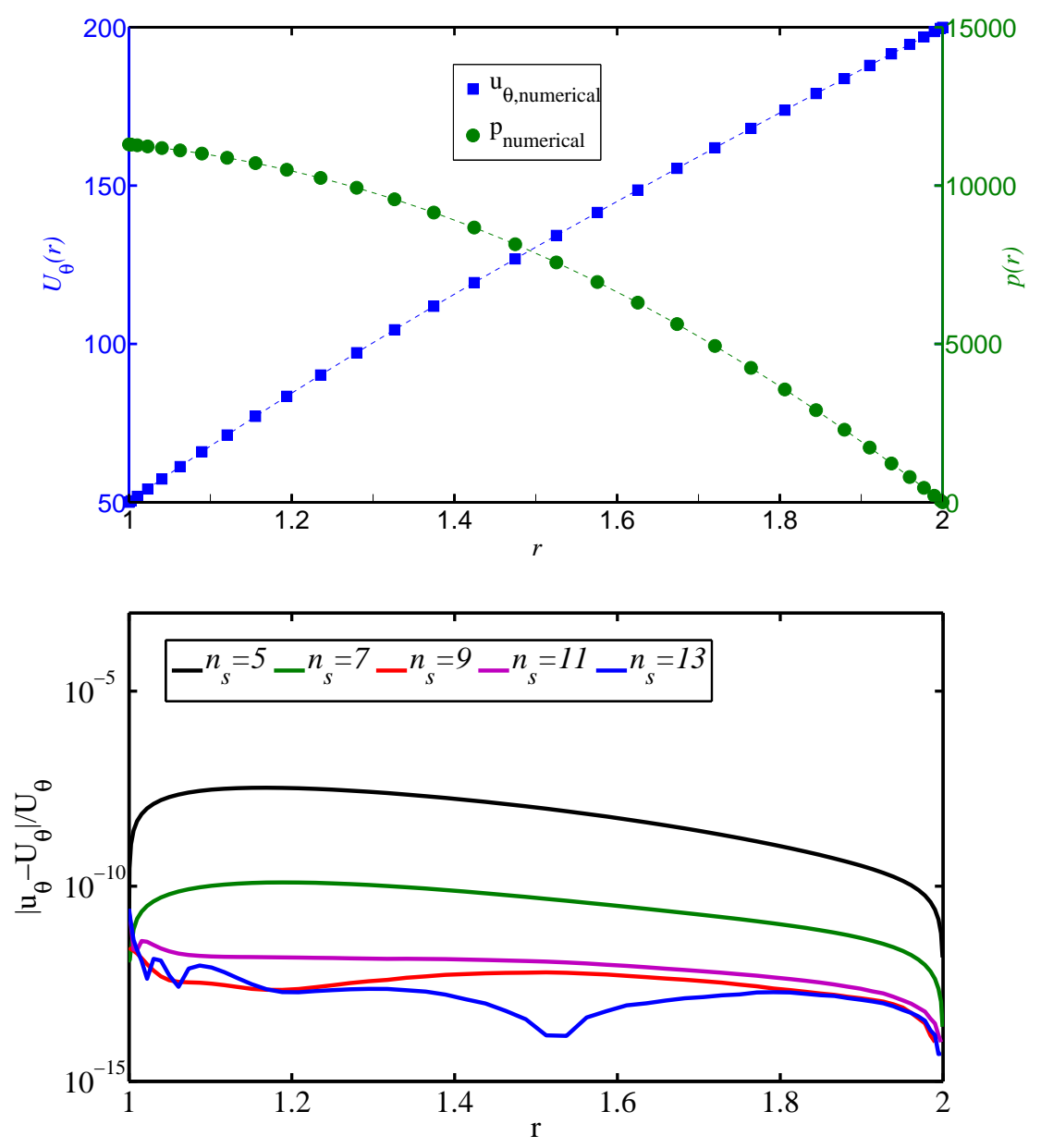

Figure 2.2: Laminar Couette flow. (top) The numerical and theoretical (dashed lines) streamwise velocity profile (blue squares) and pressure field (green circles). (bottom) The local relative error $\epsilon_{u}$ as a function of $r$. Here $\Gamma=2$ and $k_{\theta}=2$, with a resolution of $\left(n_{r}, n_{\theta}, n_{z}\right)=(64,8,8)$. In the radial direction Chebyshev points are used, $\alpha \rightarrow 0$.

To measure the global error, we integrate the local error $\epsilon_{u}$ over the radial direction, $\mathcal{E}_{u}=\int_{r_{i}}^{r_{o}} \epsilon_{u} r d r$, which is pl otted in Fig. 2.3 as a function of $n_{r}$ and the parameter $\alpha$. In the left figure, $\mathcal{E}_{u}$ scales as a power law with $n_{r}$ for bo th $\alpha=0$ and $\alpha=0.5$. The power exponent is fitted to be about -11, which is even better than as is expected from the 9-points-stencil FD scheme. The right figure shows that the error is minimized for $\alpha \simeq 0.5$ and that below 0.5 the errors are almost at the same level and thus are all acceptable at this Re. Except that $n_{r}$ is varied in the left figure, the rest resolution is the same as in above figure.

\subsubsection{Time-dependent flow and slip velocity at walls}

The onset of stability in the case of stationary outer cylinder $\left(R e_{o}=0\right)$ has been verified at two different radius ratios $\eta=0.5,0.95$. As the Reynolds number of the 

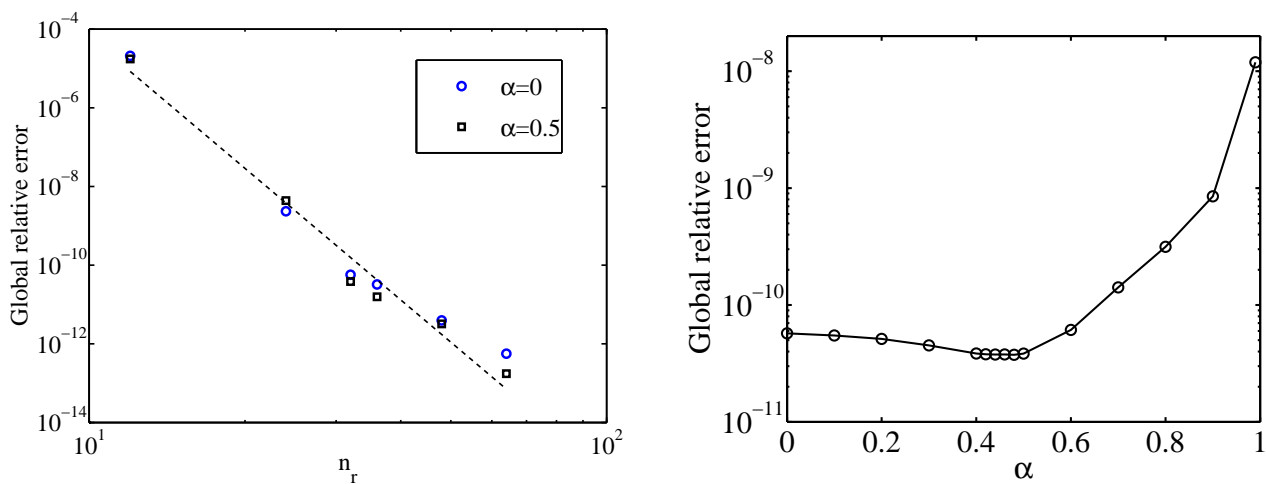

FiguRE 2.3: The global relative error $\mathcal{E}_{u}$ as a function of (left) $n_{r}$ and (right) $\alpha$. The dashed line in the left figure is the power fit with an exponent about -11 . The stencil length is $n_{s}=9$. The resolution $\left(n_{r}, n_{\theta}, n_{z}\right)=(32,8,8)$, except $n_{r}$ in the left figure.

inner Cylinder increases beyond a certain value, the laminar Couette flow gives way to Taylor vortices (see Fig. 2.4). In our simulations, this critical Reynolds number is estimated by measuring the exponential growth rate of the perturbed kinetic energy, which vanishes at the critical point. It turns out that $R e_{i}^{\text {crit }} \in[68,68.5]$ for $\eta=0.5$ and $R e_{i}^{c r i t} \in[184.5,186]$ for $\eta=0.95$, which are consistent with the values reported in previous publications (see Table 2.1).

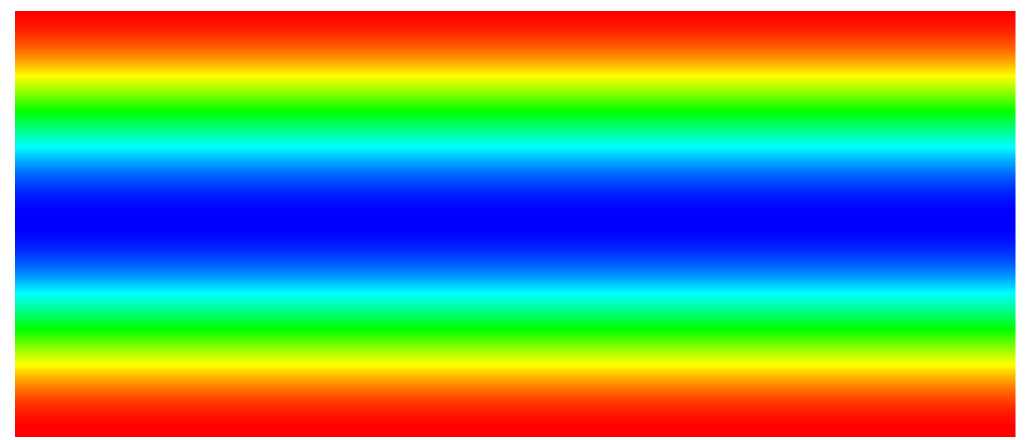

Figure 2.4: Contour plot of the mid-gap streamwise velocity in the middle $(\theta, z)$ plane for Taylor vortices at $R e_{i}=182, R e_{o}=0$.

\begin{tabular}{ccc}
\hline \hline$\eta$ & $R e_{i}^{\text {crit }}$ & reported value $[100]$ \\
\hline 0.5 & $68.2 \pm 0.3$ & 68.2 \\
0.95 & $185 \pm 0.5$ & $\in[184,186]$ \\
\hline \hline
\end{tabular}

TABLE 2.1: Critical Reynolds number at inner cylinder for the appearance of Talor vortices: $\eta=0.5$ and $\eta=0.95$.

Time-dependent periodic flow was computed at $R e_{i}=458.1, R e_{o}=0, \eta=0.868$. The axial length was chosen as $\Gamma=2 \pi / k_{z}=2.4$ and $k_{\theta}=6$ to compare to the experimental observations of King et al. [101] and numerical simulations of Marcus [91]. At these parameter values the flow is characterized by wavy Taylor vortices with azimuthal wavenumber 6, as shown in Fig. 2.5. Wavy Taylor vortices are a relative equilibrium: 
they consist of a constant pattern rotating as a solid at a constant wave speed. Marcus [91] notes: 'A test that is more sensitive than the comparison of torques is the comparison of the numerically computed wave speed with the experimentally observed wave speed'. We performed this test with spatial resolution $\left(n_{r}, n_{\theta}, n_{z}\right)=32 \times 32 \times 32$ and time-step size $\Delta t=2 \times 10^{-5}$. The wave speed normalized by the rotation speed of the inner cylinder was accurately computed with a rigorous method ba sed on Brent's minization algorithm (for details see Appendix A). Our result the wave speed $c=0.34432$, with the pattern rotating at about one-third of the speed of inner the cylinder, agrees to all decimal places given in [101]. The same result was reproduced at higher resolutions and on various HPC platforms.

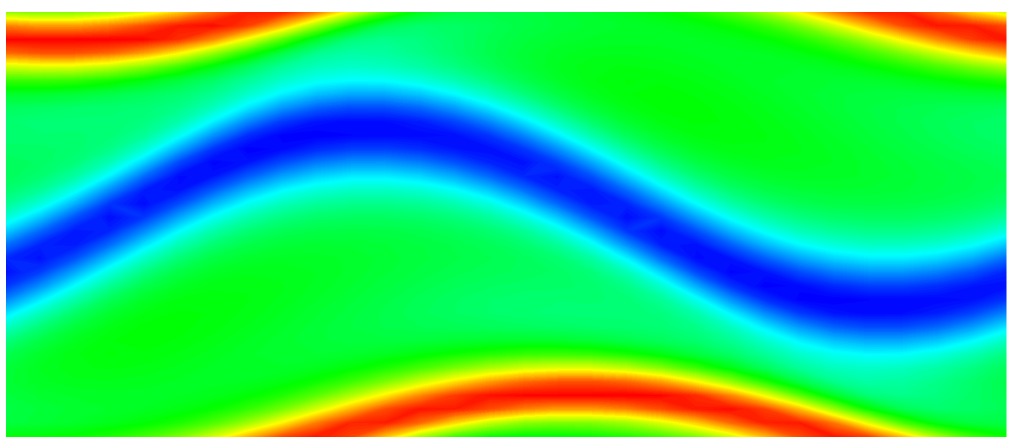

Figure 2.5: Contour plots of the streamwise velocity in the middle $(\theta, z)$ plane for wavy Taylor vortices. The outer cylinder is stationary, whereas the inner cylinder rotates with $R e_{i}=458.1$. The geometrical parameters are $\eta=0.868$ and $\Gamma=2.4$ and only one sixth of the circle $\left(k_{\theta}=6\right)$ was used in the simulations and is displayed here.

We further examined the tangential velocity slip at the cylinders. In the projection scheme we employed, the incompressibility constraint $\nabla \cdot \mathbf{u}=0$ is discretely fulfilled by construction, in that the Poisson equation for $\phi$ in 2.3 is derived by applying the divergence-free condition. However, the velocities at the inner and outer cylinders slip by an amount of $|\nabla \phi|=\mathcal{O}\left(\Delta t^{3}\right)$ after the correction step [93, 102]. We evaluated the L2-norm of the tangential velocity slip at the inner cylinder, $\int_{\theta} \int_{z} \sqrt{\left.\left(\left(u_{\theta}-R e_{i}\right)^{2}-u_{z}^{2}\right)\right|_{r=r_{i}}} d \theta d z$. In Fig. 2.6 the relative velocity slip, i.e. slip velocity normalized with $R e_{i}$, is shown as a function of $\Delta t$ for several radial resolutions $n_{r}$ (see Fig. 2.6). For the lowest resolution $n_{r}=32$ the curve rapidly levels off, indicating that spatial-discretization errors domina te over temporal errors. Note that with the largest time-step size allowed for stability and lowest resolution we already obtain five digits in the accuracy of $c$. As $n_{r}$ is increased the slip velocity decreases and its scaling gradually approaches a power law, here with an exponent of approximately 2.5. Improving the resolution in $\theta$ and $z$ directions does not change the scaling and the reason why it deviates from the expected value of 3 is not clear. Nevertheless, we can conclude that in typical simulations the dominating source of error comes from the spatial discretization. The largest possible time-step size yield s already very accurate results in the solution and very small slip velocities. 


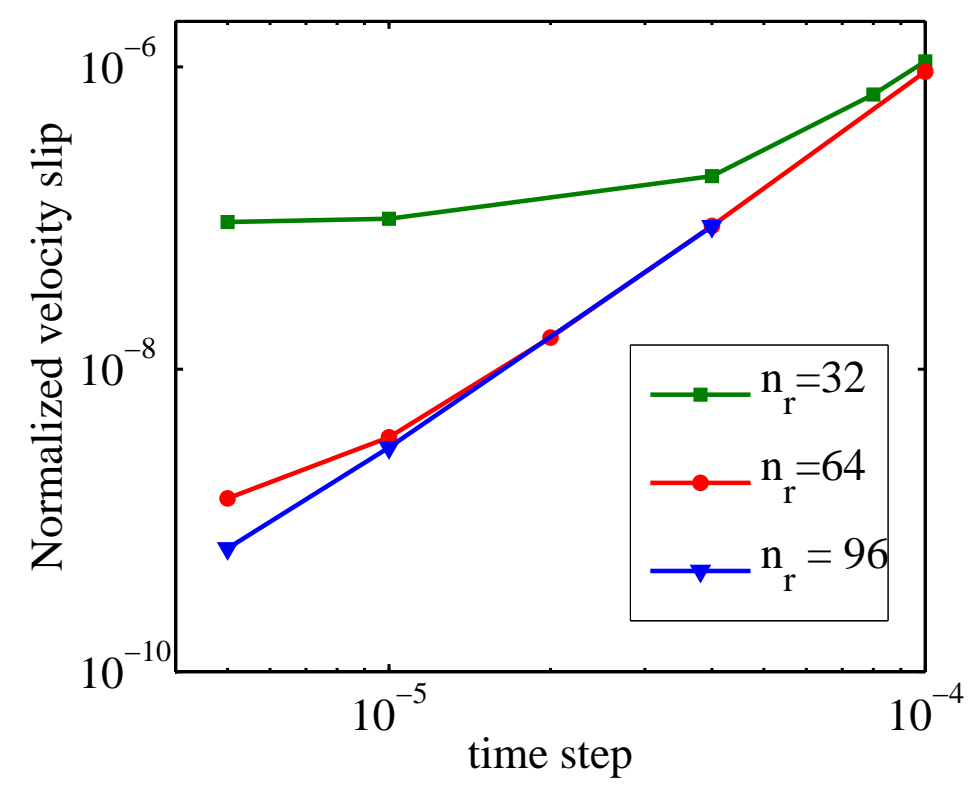

FiguRE 2.6: Normalized velocity slip at the inner cylinder against time-step size $\Delta t$ for different $n_{r}$. The parameters are the same as in Figure 2.4. The spatial resolution in axial and azimuthal directions is $\left(n_{\theta}, n_{z}\right)=(32,32)$

\subsubsection{Localized turbulence at moderate $\mathrm{Re}$}

Localized turbulence, interspersed in the surrounding laminar flow, is a typical feature of transitional Reynolds numbers in shear flows. The turbulent stripe pattern found in the counter-rotating Taylor-Couette flow in the narrow-gap limit is an example. We obtained this pattern at $R e_{i}=680, R e_{o}=-680$ with $\eta=0.993$. The time-step size was $\Delta t=2 \times 10^{-5}$ and the domain size in the axial and azimuthal directions was $\Gamma=50$ and $k_{\theta}=179$. The $\theta$-direction in our computational domain is tilted with an angle of $24^{\circ}$ to the streamwise direction (for details see [103]). We tested the probability distributions of the splitting times of turbulent stripes reported in [103], which were obtained by using the spectral Petrov-Galerkin code of Meseguer et al. [90]. We here used $n_{r}=32$ in the radial direction, whereas Shi et al. [103] used modified Chebyshev polynomials of degree up to 26 . In both cases the azimuthal and axial resolutions are $n_{\theta}=48$ and $n_{z}=640$, respectively. The exponential distributions of splitting times obtained by both codes are statistically equivalent (see the inset in Fig. 2.7): our computed decay rate of the exponential distributions is well within the $95 \%$ confidence interval about the decay rate reported in [103].

\subsubsection{Turbulent flow at high Re}

The robustness of the code was further validated at high Reynolds numbers in the linearly unstable regime, where the flow is fully turbulent. Here we computed the 


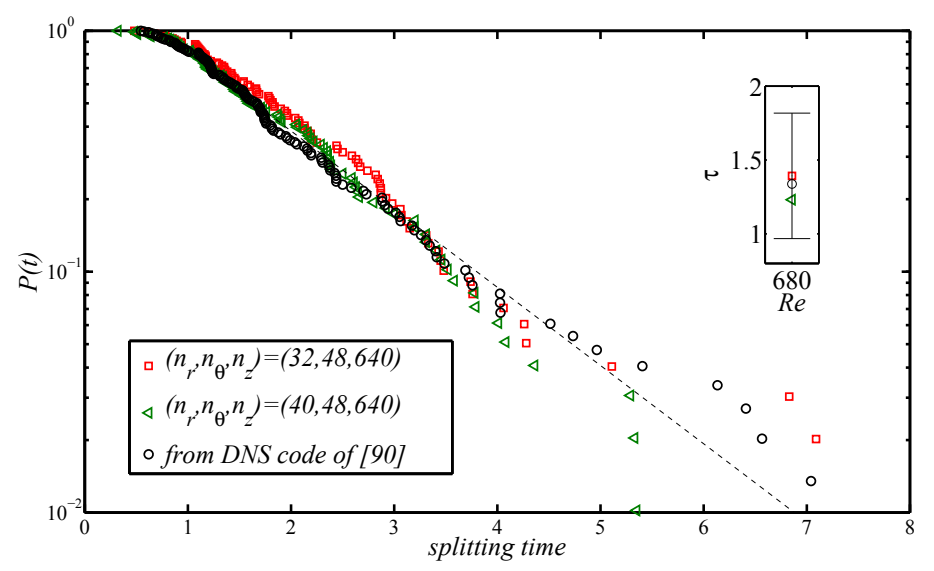

FIGURE 2.7: Probability distributions of the splitting time of single turbulent stripe at $R e_{i}=680, R e_{o}=-680$ and at $\eta=0.993$. The black data set was obtained with the spectral Petrov-Galerkin code of Meseguer et al. [90] and the red one with the new code with $n_{r}=32$. In both cases the azimuthal and axial resolutions are $n_{\theta}=48$ and $n_{z}=640$, respecitvely. The red curve is from the present code. Inset: characteristic splitting time estimated by the sample mean, with the error bar corresponding the $95 \%$ confidential interval.

global torque exerted by the fluid on the inner and outer cylinders, which characterizes the turbulence intensity and the transport of angular momentum [87]. The tests were done at $R e_{i}=8000$ and stationary outer cylinder with $\eta=0.5, \Gamma=2 \pi / k_{z}=\pi$ and $k_{\theta}=2$. The time-step size is $\Delta t=2 \times 10^{-7}$. As is shown in Fig. 2.8(top), the quasi-Nusselt number $N u_{\omega}$ [87], which is the torque normalized by the torque of the laminar flow, converges to 8.815 at the resolution $\left(n_{r}, n_{\theta}, n_{z}\right)=(128,192,320)$. This value agrees very well to the value of 8.816 recently reported by Brauckmann and Eckhardt [87], who also used the spectral Petrov-Galerkin code of Meseguer et al. [90]. The temporal fluctuation of the Nusselt number obtained with the highest resolution is shown in the bottom figure. At this $R e$, we also examined the influence of the radial node distribution by varying the parameter $\alpha$ in Eq. 2.10 . Three runs with $\alpha=0,0.5,0.99$ were done, and all three render $N u_{\omega}=8.81 \pm 0.05$. The maximum time-step size is $\Delta t \approx 3 \times 10^{-7}$ in all cases and it is independent of $\alpha$. This is explained by the fact that the CFL number is highest in the azimuthal direction. Thus at this Reynolds number the Chebyshev node distribution does not impose a restriction in the time-step, as one may have expected.

Another test run was performed at $R e_{i}=10^{5}, R e_{o}=79685$ and at $\eta=0.71$. To the best of our knowledge, no DNS at such a high Re have been reported for TaylorCouette flow. We used $k_{\theta}=16$ and axial length $\Gamma=0.5$, with a spatial resolution $(1152 \times 384 \times 384)$ and time step $\Delta t=1 \times 10^{-9}$. The initial condition at $t=0$ is taken from the optimal initial perturbation which gives the maximal transient energy growth [104] supplemented with very small three-dimensional noise. Fig. 2.9] shows the $3 \mathrm{D}$ contour plot of the streamwise vorticity, $\omega_{\theta}=\partial_{z} u_{r}-\partial_{r} u_{z}$, at $t=5 \times 10^{-4}(\simeq 3.3$ 
Chapter 2. A highly efficient parallel DNS code nsCouette
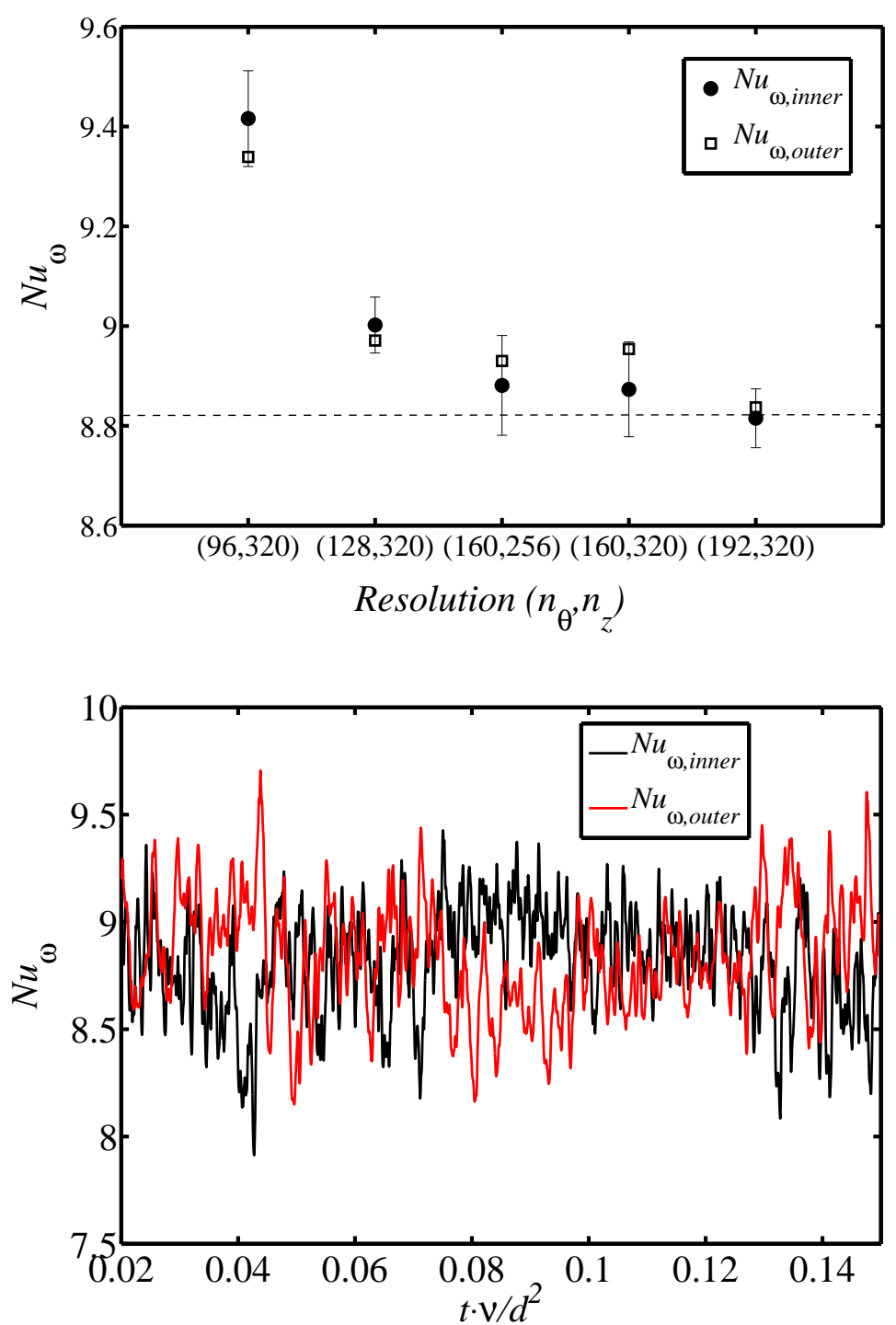

FiguRe 2.8: Top: Quasi-Nusselt number at $R e_{i}=8000, R e_{o}=0, \eta=0.50, k_{z}=\pi$ and $k_{\theta}=2$, as a function of the azimuthal and axial resolutions for $n_{r}=128$. The dashed line corresponds to the value $\left(N u_{\omega} \simeq 8.816\right)$ reported in 87 ]. The error bars indicate the $95 \%$ confidential interval. Bottom: The temporal fluctuation of the quasi-Nusselt number at the highest resolution $\left(n_{r}, n_{\theta}, n_{z}\right)=(128,192,320)$.

cylinder rotations) which illustrates a transiently turbulent flow state. The research is still ongoing and will be disseminated in future publications. We expect that the results will contribute to clarify the role of pure hydrodynamic turbulence, if any, in astrophysical disks [78]. 


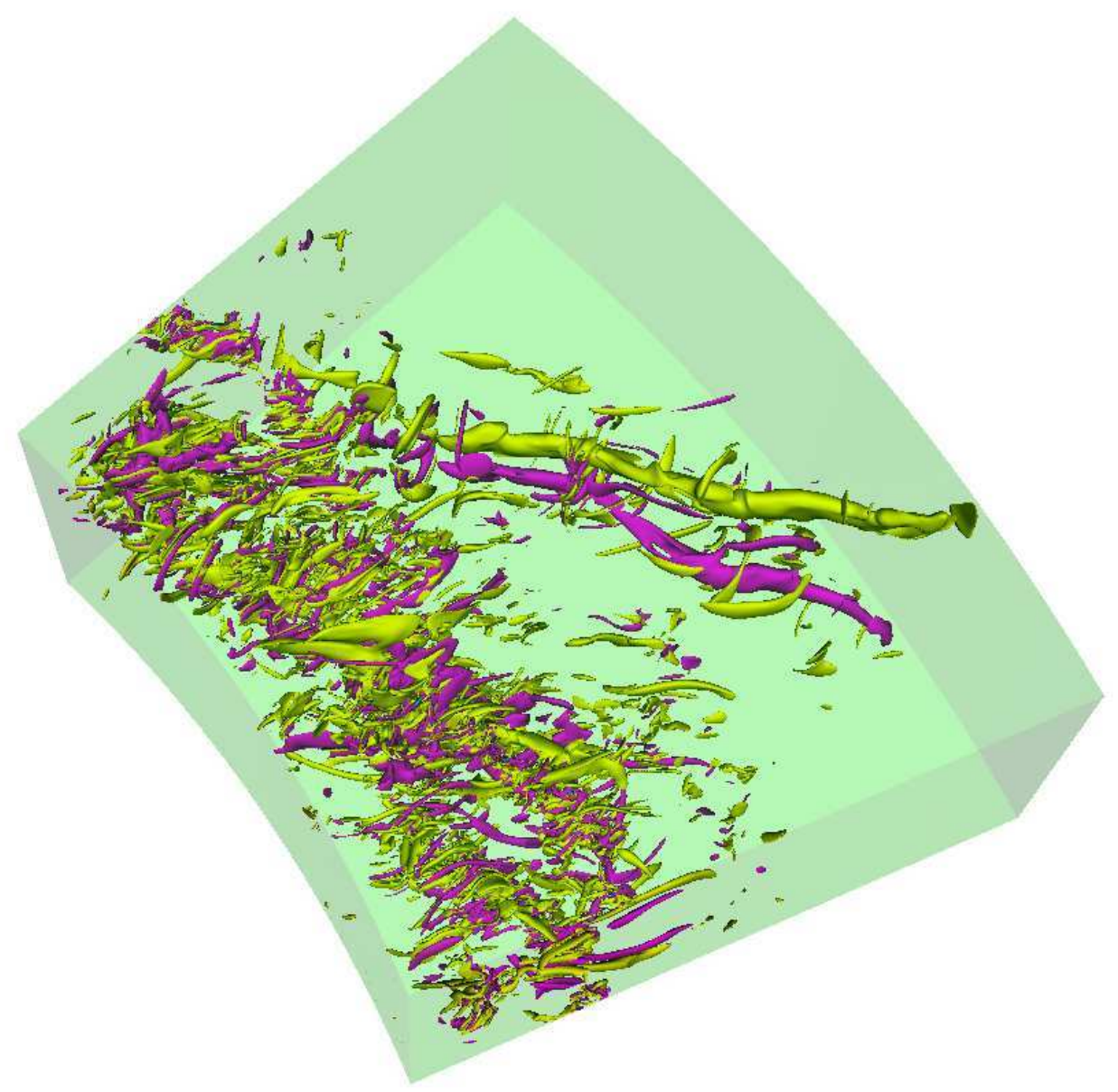

Figure 2.9: Isosurfaces of the streamwise vorticity in the quasi-Keplerian regime at $R e_{i}=10^{5}, R e_{o}=79685, \eta=0.71, \Gamma=0.5$ and $k_{\theta}=16$. The resolution is $\left(n_{r}, n_{\theta}, n_{z}\right)=(1152,384,384)$.

\subsection{Computational efficiency 2}

\subsubsection{Benchmark setup}

In this section we report benchmarks results obtained with the new code, using up to 8192 processor cores of an IBM iDataPlex compute cluster with Intel Sandy-Bridge processors and InfiniBand (FDR 14) interconnect. Each shared-memory compute node hosts two Intel Xeon E5-2670 eight-core processors (CPUs) with a clock frequency of 2.6 GHz. We employ Intel compilers (version 12.1), the Intel Math Kernel Library (MKL 10.3) and the FFTW library (version 3.3.2 with AVX kernels enabled).

\footnotetext{
${ }^{2}$ The work in this section is mainly done by Dr. Markus Rampp and is put here for the reason of consistency.
} 
We have performed two strong scaling studies, i.e., the scaling of the runtime with the number of cores for a fixed problem size. Two different, representative setups were considered:

a) a "SMALL" setup with number of grids points $\left(n_{r}, n_{\theta}, n_{z}\right)=(32,384,640)$. This setup is used to investigate the localized turbulence at the transitional stage ( $R e \sim \mathcal{O}\left(10^{2}\right)$ ), where the structures inside the turbulence are relatively large. The probability distributions of the splitting time of localized turbulent stripe mentioned in Section 2.5 are obtained at this resolution level.

b) a "LARGE" setup with number of grids points $\left(n_{r}, n_{\theta}, n_{z}\right)=(512,256,1024)$. This resolution is representative of our ongoing studies of hydrodynamic turbulence in Taylor-Couette flows with quasi-Keplerian velocity profiles at Reynolds numbers up to $\mathcal{O}\left(10^{5}\right)$.

\subsubsection{Benchmark results and discussion}

Fig. 2.10 provides an overview of the strong scalability of the hybrid code. Different colors and symbols are used to distinguish runs which use different numbers of MPI tasks $\left(N_{\text {tasks }}\right)$ and OpenMP threads ( $\left.N_{\text {threads }}\right)$. The total number of processor cores is given by $N_{\text {cores }}=N_{\text {tasks }} \cdot N_{\text {threads }}$.

For both setups we observe good scalability up to the maximum number of cores our parallelization scheme admits on this compute platform, i.e., $\quad N_{\text {cores }}=32 \cdot 16=512$ for the SMALL setup, and $N_{\text {cores }}=512 \cdot 16=8192$ for the LARGE setup. Beyond a number of 8 threads per MPI task the scalability curves markedly level off. The code, however, still delivers a parallel efficiency of more than 0.5 , which is commonly considered as the minimum for acceptable resource usage.

For the SMALL setup (Fig. 2.10, left) we show that up to a number of 8 threads per MPI task the run times for a given number of cores are virtually the same, independent of the distribution of the resources to MPI tasks and OpenMP threads (compare the green and the red symbols at $N_{\text {cores }}=32$, or the red and the blue symbols at $N_{\text {cores }}=$ 128). This indicates that the efficiency of our coarse-grained OpenMP parallelization is almost the same as the explicit, MPI-based domain decomposition, thus demonstrating the additional gain in flexibility of our hybrid approach compared to a plain MPI parallelization. Moreover, as the results for the LARGE setup (Fig. 2.10, right) show, it can even be more efficient to use less than the maximum of $n_{r}$ MPI tasks for a given number of cores and utilize the resources with OpenMP threads (compare the green and the red symbols at moderate core counts). This is due to the fact that a lower number of MPI tasks per node reduces the amount of inter-node MPI communication 
(specifically the MPI_Alltoall communication pattern for the global transpositions) and hence network congestion. Notably, for the LARGE setup, the hybrid code shows nearly perfect linear scaling between 128 and 2048 cores.

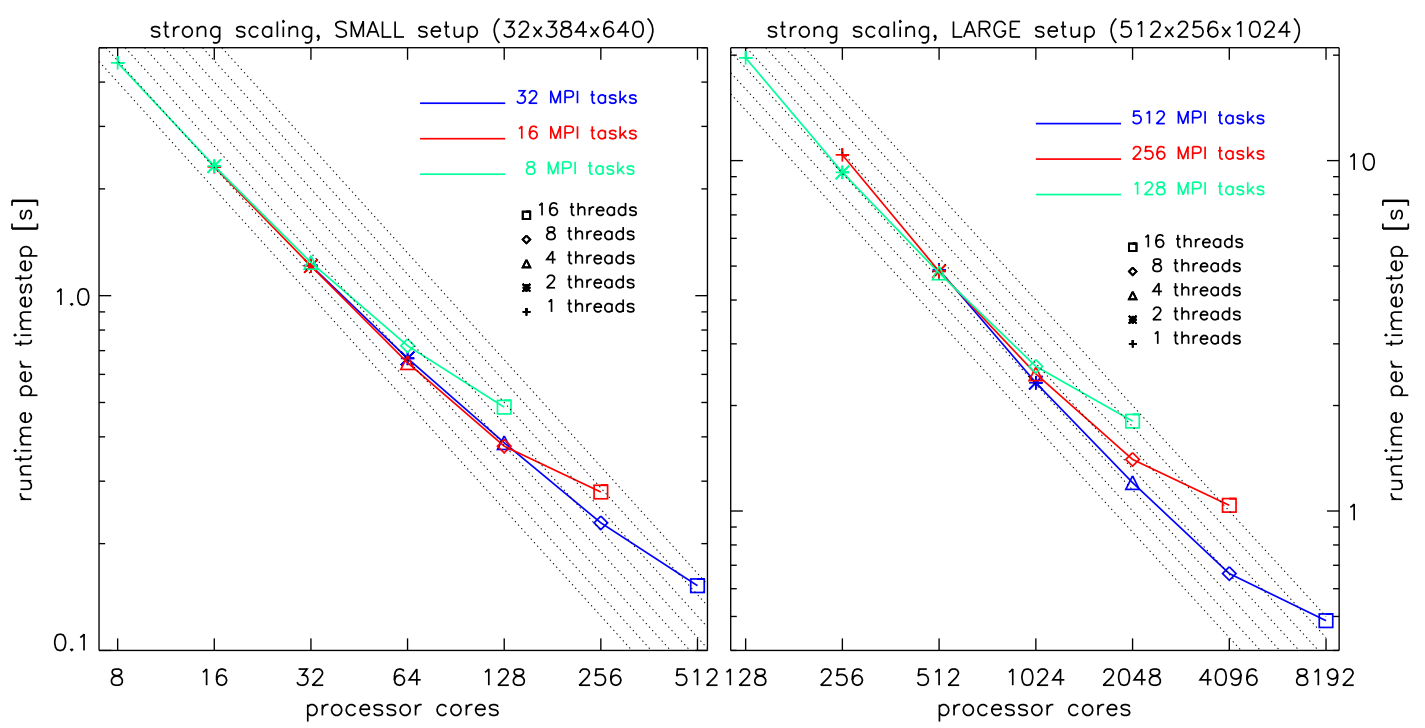

FiguRE 2.10: Runtime per time step for the SMALL setup (left panel) and for the LARGE setup (right panel) as a function of the number of cores, $N_{\text {cores }}=N_{\text {tasks }}$. $N_{\text {threads. }}$. Different colors and symbols are used to distinguish runs with different numbers of MPI tasks $\left(N_{\text {tasks }}\right)$ and OpenMP threads $\left(N_{\text {threads }}\right)$, respectively. The slope of an ideal scaling curve is indicated by dotted lines.

The details on the absolute run times and the parallel efficiencies of the whole code (the bottom row) as well as the individual parts of the algorithm (cf. Section 2.3) are listed in Table 2.2. The first column, which corresponds to a plain MPI-parallelization using the maximum number of tasks $\left(N_{\text {tasks }}=n_{r}\right)$ for the given setup, is assigned an efficiency of 1.0, by definition. The code reaches a floating-point performance of about 16 GFlop/s per compute node which is roughly $5 \%$ of the nominal peak performance.

For the SMALL setup (the upper part of Table 2.2) we observe perfect OpenMP efficiency up to 8 threads (which are pinned to the 8 physical cores of single CPU socket) per MPI-task for the pressure and velocity predictor steps, the corrector step, and also the matrix-vector multiplication in the nonlinear part. When using all 16 cores of a shared-memory node with a single MPI task one notices a slight degradation in OpenMP efficiency due to memory-bandwidth limitations and NUMA effects. The overall parallel efficiency (the bottom row) can be considered as very good up to 128 cores, but gets increasingly bounded by the global transposition (MPI_Alltoall communication) and the limited parallelism in the nonlinear part.

For the LARGE setup (the lower part of Table 2.2), where the highly scalable linear parts and the matrix-vector multiplication still contribute more than $60 \%$ to the total runtime on 4096 processor cores, the code maintains an excellent parallel efficiency 
Chapter 2. A highly efficient parallel DNS code nsCouette

\begin{tabular}{|c|c|c|c|c|c|c|c|c|c|c|}
\hline \multicolumn{11}{|c|}{$\overline{\text { SMALL setup }(32,384,640)}$} \\
\hline cores $\left(N_{\text {threads }}\right)$ & \multicolumn{2}{|c|}{$32(1)$} & \multicolumn{2}{|c|}{$64(2)$} & \multicolumn{2}{|c|}{$\overline{128(4)}$} & \multicolumn{2}{|c|}{$256(8)$} & \multicolumn{2}{|c|}{$512(16)$} \\
\hline & $T_{1}[\mathrm{~s}]$ & $\eta$ & $T_{2}[\mathrm{~s}]$ & $\eta$ & $T_{4}[\mathrm{~s}]$ & $\eta$ & $T_{8}[\mathrm{~s}]$ & $\eta$ & $T_{16}[\mathrm{~s}]$ & $\eta$ \\
\hline nonlinear & 0.760 & $100 \%$ & 0.435 & $87 \%$ & 0.265 & $72 \%$ & 0.161 & $59 \%$ & 0.105 & $45 \%$ \\
\hline$p$ prediction (2) & 0.084 & $100 \%$ & 0.042 & $99 \%$ & 0.021 & $100 \%$ & 0.011 & $97 \%$ & 0.006 & $84 \%$ \\
\hline $\mathbf{u}$ prediction $(3)$ & 0.218 & $100 \%$ & 0.109 & $99 \%$ & 0.055 & $98 \%$ & 0.028 & $96 \%$ & 0.016 & $83 \%$ \\
\hline correction (4) & 0.089 & $100 \%$ & 0.044 & $101 \%$ & 0.022 & $101 \%$ & 0.011 & $99 \%$ & 0.006 & $90 \%$ \\
\hline complete step & 1.217 & $100 \%$ & 0.666 & $91 \%$ & 0.385 & $79 \%$ & 0.229 & $66 \%$ & 0.152 & $50 \%$ \\
\hline \multicolumn{11}{|c|}{ LARGE setup $(512,256,1024)$} \\
\hline cores $\left(N_{\text {threads }}\right)$ & \multicolumn{2}{|c|}{$512(1)$} & \multicolumn{2}{|c|}{$1024(2)$} & \multicolumn{2}{|c|}{$2048(4)$} & \multicolumn{2}{|c|}{$4096(8)$} & \multicolumn{2}{|c|}{$8192(16)$} \\
\hline & $T_{1}[\mathrm{~s}]$ & $\eta$ & $T_{2}[\mathrm{~s}]$ & $\eta$ & $T_{4}[\mathrm{~s}]$ & $\eta$ & $T_{8}[\mathrm{~s}]$ & $\eta$ & $T_{16}[\mathrm{~s}]$ & $\eta$ \\
\hline nonlinear & 1.20 & $100 \%$ & 0.67 & $90 \%$ & 0.33 & $91 \%$ & 0.20 & $75 \%$ & 0.19 & $39 \%$ \\
\hline$p$ prediction (2) & 1.12 & $100 \%$ & 0.49 & $114 \%$ & 0.25 & $112 \%$ & 0.13 & $108 \%$ & 0.08 & $88 \%$ \\
\hline $\mathbf{u}$ prediction $(3)$ & 1.38 & $100 \%$ & 0.61 & $113 \%$ & 0.31 & $111 \%$ & 0.17 & $101 \%$ & 0.11 & $78 \%$ \\
\hline correction (4) & 1.12 & $100 \%$ & 0.52 & $108 \%$ & 0.28 & $100 \%$ & 0.15 & $93 \%$ & 0.09 & $78 \%$ \\
\hline complete step & 4.88 & $100 \%$ & 2.32 & $106 \%$ & 1.20 & $102 \%$ & 0.66 & $92 \%$ & 0.49 & $62 \%$ \\
\hline
\end{tabular}

TABLE 2.2: Runtime per time step, $T_{n}$ and parallel efficiency $\eta$ of the OpenMP parallelization as a function of the number $N_{\text {threads }}$ of OpenMP threads per MPI task, using the maximum number of 32 MPI tasks for the SMALL setup, and 512 MPI tasks for the LARGE setup, respectively. Parallel efficiency is conventionally defined as $\eta:=T_{1} /\left(n \cdot T_{n}\right)$ with $n=N_{\text {threads. }}$. Different rows show the contributions of the individual algorithmic steps (numbering in brackets chosen according to Section 2.3) to the total runtime of a complete time step (the bottom row).

of $92 \%$. Even up to 8192 cores an acceptable parallel efficiency of more than $60 \%$ is achieved. Computing times on the order of $0.5 \mathrm{~s}$ per time step enable us to perform highly resolved simulations (e.g. of Keplerian flows which require on the order of a million time steps) within a couple of days.

\subsection{Discussion and summary}

With the motivation of exploring high-Reynolds-number turbulent flows, we have developed a highly efficient parallel DNS code for Taylor-Couette flows. The incompressible Navier-Stokes equations in cylindrical coordinates are solved in primitive variables by using an improved projection method proposed by Hugues et. al. [93], which is secondorder accurate in both pressure and velocity. This method leads at each time step to the solution of five linear differential equations, either of Poisson or of Helmholtz type, which simplifies significantly the programming of the code. For the spatial discretization, we use a combination of Fourier spectral in axial and azimuthal directions and finite differences in the radial direction, which allow the use of tailored stretched grids. The computing cost scales linearly with the number of grid points in each direction.

In order to reach higher Reynolds numbers and to take full advantage of the modern HPC facilities, the code is parallelized by a hybrid MPI-OpenMP strategy, combining the simplicity of a MPI-based one-dimensional "slab" domain decomposition in 
Fourier space with efficient exploitation of the remaining coarse-grained parallelism by OpenMP threading. Compared to a flat MPI-parallelization, the hybrid code maps more naturally to the current multi-node, multi-core architectures and, most importantly, reduces inter-node communications, which improves the overall efficiency and scalability. The strong scaling study which was performed with scientifically relevant setups shows a very good scalability of the code up to $\mathcal{O}\left(10^{4}\right)$ cores. This allows to perform simulations with much higher resolutions than previously possible. With the current HPC technology, this code pushes the achievable Re to the order of magnitude of $\mathcal{O}\left(10^{5}\right)$ in DNS of Taylor-Couette flow, which therefore opens up the possibility to study quasi-Keplerian flows at experimentally relevant parameters.

The new code has been shown to be very accurate in various regimes: laminar Couette flow, wavy vortices, transitional and turbulent flow at high Re. With the high efficiency of the hybrid parallel scheme, this code possesses great potential to explore the turbulent TC flows in a much broader parameter space. 
"Big WHIRLS HAVE LITTLE WHIRLS

THAT FEED ON THEIR VELOCITY;

AND LITTLE WHIRLS HAVE LESSER WHIRLS

AND SO ON TO VISCOSITY."

LEWIS FRY RichaRdson (1881 - 1953)

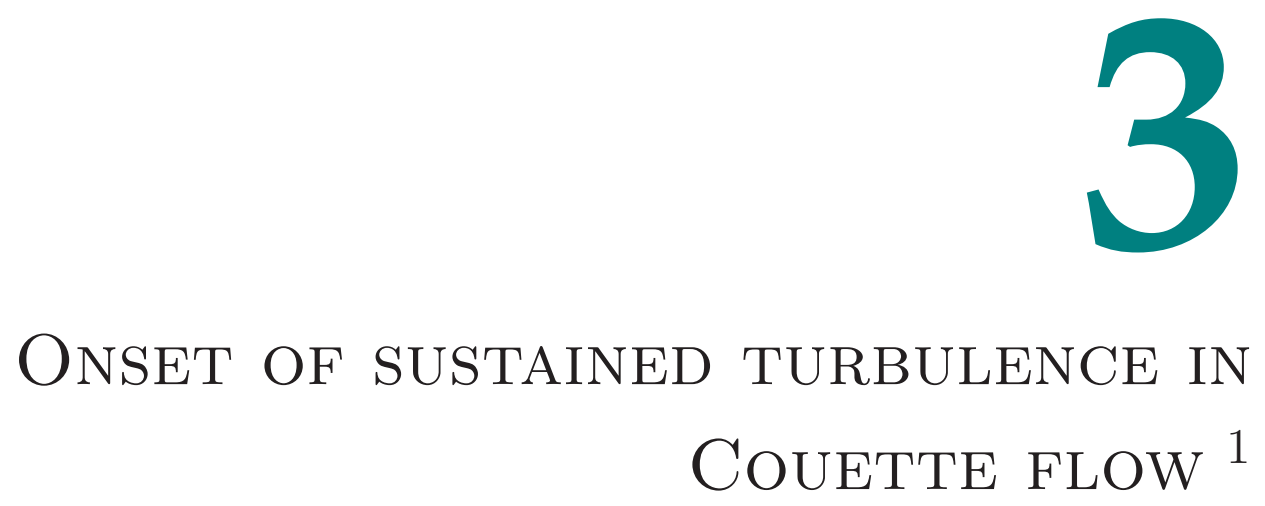

\subsection{Introduction}

Turbulence often arises despite linear stability of the laminar flow and the nature of this transition has remained unresolved for over a century [1]. Prominent examples are pipe, channel and Couette flows. Here finite amplitude perturbations can lead to sufficiently strong distortions of the base flow, that for large enough Reynolds numbers cause transition to turbulence. At moderate Re turbulent structures are localized and are commonly referred to as spots or stripes in Channel and Couette flow, and puffs in pipe flow. Individual localized structures are of transient nature [16, 18, 45, 105] but they can also temporarily grow and seed new spots in their vicinity, overall resulting in complex spatial-temporal dynamics. Figure 3.1 shows the growth process of a turbulent spot in PCF, leading to a statistically steady state, a turbulent stripe. The turbulent stripes can further spread through the splitting events (more details in [106]). As shown for pipe flow, this spreading rate increases with $R e$, eventually outweighing the decay [12]. It has been argued that this mechanism causes a phase transition in the thermodynamic limit from transient to sustained turbulence [60]. As Re surpasses the critical point, turbulence can overall survive due to the spatial spreading. The

\footnotetext{
${ }^{1}$ The first part of the content in this Chapter is mainly from the paper: L. Shi, M. Avila, B. Hof, Scale Invariance at the Onset of Turbulence in Couette Flow, Phys. Rev. Lett., 110, 204502 (2013).
} 
observed dynamics bears resemblance to contact process such as directed percolation (DP) 11]. In this analogy laminar flow corresponds to the absorbing passive state and turbulence to the active one. However, for pipe flow [12] the phase transition has not been characterized directly, but has only been inferred from the statistical behaviour (mean splitting and decay times) of individual spots. The main difficulty in pipe flow is that the relevant time scales of the relevant processes are extremely large, putting a study of correlation exponents presently beyond reach. Here it is reported a numerical (DNS) study of another fundamental shear flow, plane Couette flow (PCF) where the fluid is sheared between two sliding plates (see Figure 3.2(left)). As will be shown below, the time scales close to the critical point are much smaller than those in pipe flow. Hence size distributions and turbulent fractions can be determined close to criticality, which provides a unique opportunity to characterize the phase transition.

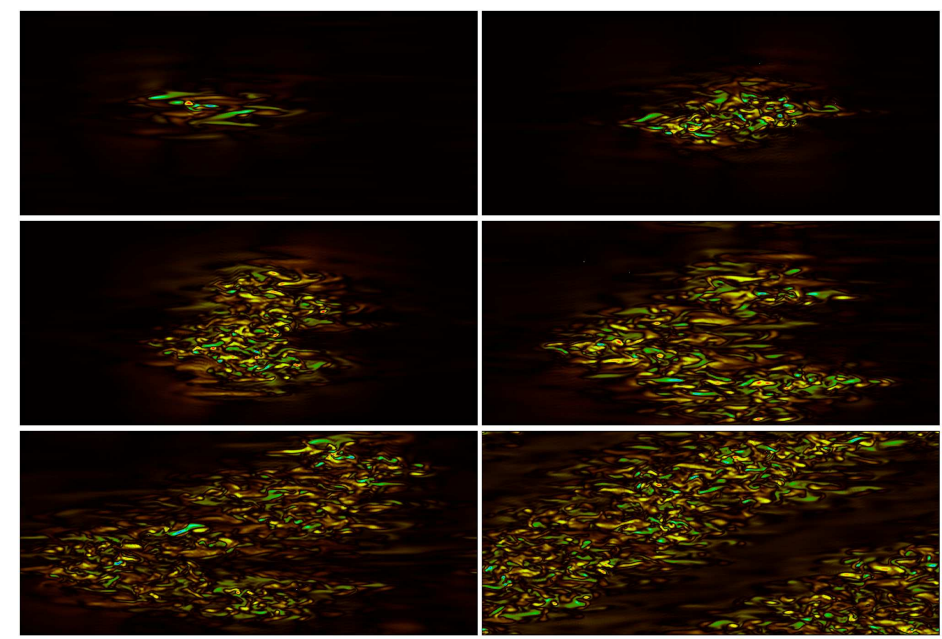

Figure 3.1: Growth of the turbulent spot in PCF at $R e=350$, based on half gap distance. Streamwise vorticity field in the mid-plane is plotted and time is advanced from top to bottom and from left to right.

\subsection{Numerical experiments}

Because of its unlimited width in the spanwise direction $\left(z^{\prime}\right.$ in Figure 3.2), PCF is spatially more complex than pipe flow. In order to simplify the spatial-temporal complexity and to enable us to resolve the long interaction times, we choose a slender computational domain inclined with the flow streamwise direction, as shown in Figure 3.2 (right). The idea of such a "tilted" computational domain was originally introduced to reduce the computing cost by Barkley \& Tuckerman [48, 107].

Simulations are carried out using the parallel code nsCouette 2. The two cylinders are chosen to counter-rotate and a radius ratio (inner to outer cylinder radius) of

\footnotetext{
${ }^{2}$ Part of the results in this Chapter are obtained by using another numerical code for Taylor-Couette flow (TCF) (see [90] for details).
} 


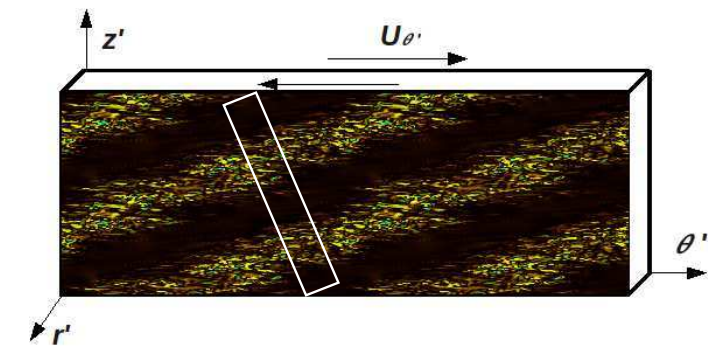

(a)

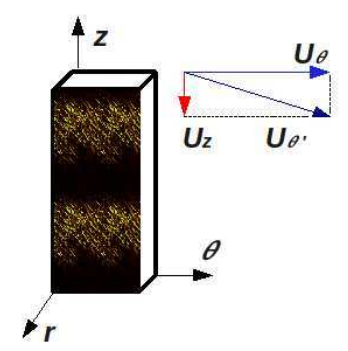

(b)

FiguRE 3.2: Schematic of the computational domain for PCF. (a) normal box; (b) "tilted" slender box [white box in (a)]. The stripe pattern in the background is a visualization of the streamwise vorticity field.

$\eta=0.993$ has been selected so that curvature effects are negligible. As shown in an earlier study [30] TCF approaches the PCF limit for these parameter settings and is linearly stable for all Re relevant to this study. By using the half gap distance $h=d / 2$ and $\tau_{h}=h / U$ (here $U$ is the magnitude of the boundary velocity) as the length and time unit, we solve numerically the incompressible Navier-Stokes equation (see section (2.3). The Reynolds number is defined as $R e=U h / \nu$. Periodic boundary conditions are imposed in the in-plane directions, while no-slip boundary conditions are applied in the wall-normal direction. The size of the computational domain is chosen sufficiently long in the $z$ direction $\left(L_{z} \times L_{\theta} \times L_{r}\right)=(100 h \times 10 h \times 2 h)$ to study the evolution of isolated stripes whereas for the study of stripe interactions $L_{z}$ is increased to $960 \mathrm{~h}$ and $1920 \mathrm{~h}$. The spatial resolution (number of grid points) for the smaller domain is $\left(n_{z} \times n_{\theta} \times n_{r}\right)=(512 \times 48 \times 32)$, the adequacy of which is checked by the one-dimensional energy spectra and by the convergence study of the statistical lifetime and splitting time distributions (see Fig. 3.3) 3. For the larger domains the axial resolution is increased to 6144 and 12288 , respectively.

\subsection{Memoryless dynamical processes}

For the investigation of the decay and splitting of individual localized turbulent stripes, uncorrelated velocity fields of single stripes were used as initial conditions, which were generated at $\operatorname{Re}=325$ and contain single turbulent stripe in the domain. Since the decay and splitting are observed to depend strongly on the initial conditions, many realizations are necessary to determine the mean lifetimes and splitting times. The Reynolds numbers of interest are in the range of $\operatorname{Re} \in[310,350]$. At each Re, 300 realizations with different initial conditions are conducted and each simulation was

\footnotetext{
${ }^{3}$ These two figures were obtained with another spectral Petrov-Galerkin code of Meseguer et al. 90], where the radial direction is discretized by the Chebyshev spectral method. The resolution is $\left(n_{z} \times\right.$ $\left.n_{\theta} \times n_{r}\right)=(512 \times 48 \times 26)$. The comparison between two codes are shown in $\S 2.5 .3$.
} 

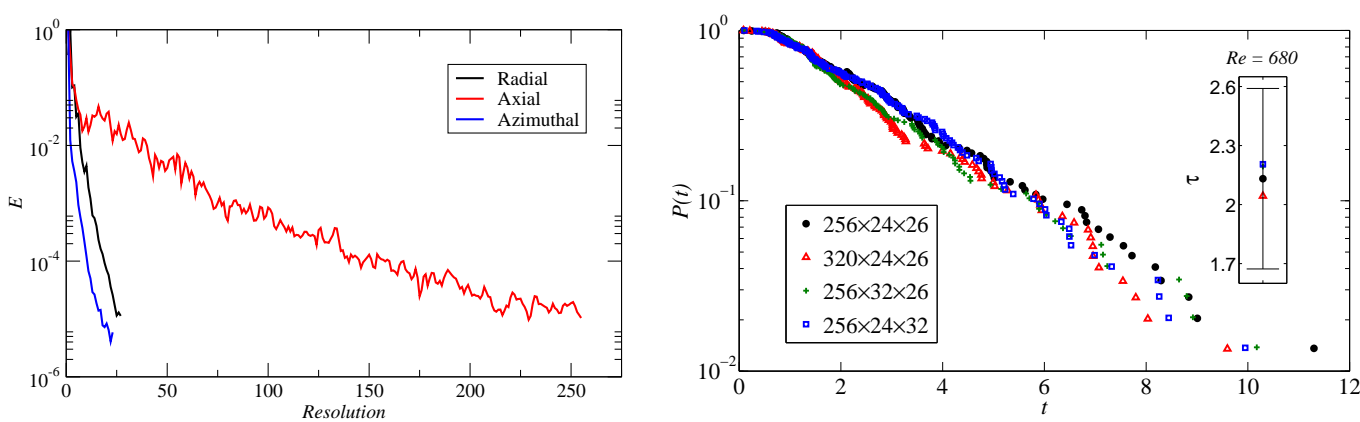

FiguRE 3.3: Numerical resolution [106]. (left) One dimensional energy spectra as function of resolution, with $\log$-scale in y-axis; (right) Probability distribution of splitting events at $R e=340$ for different resolutions. The distributions with higher resolutions are statistically equivalent. Inset is the scale parameter $\tau$ associated to the corresponding resolution. The error bar of the original resolution represents about $95 \%$ confidential interval.

run for a predefined time duration (cutoff time). If a decay or splitting occurred earlier, the run was terminated after this event. Let $N^{s}, N^{d}$, and $N^{c}$ denote the number of splitting, decaying cases and the ones reaching the cutoff time, respectively. By sorting in increasing order all the final times associated with splitting events (or splitting time), we obtain a splitting time series $\left\{t_{i}^{s}\right\}_{i=1}^{N^{s}}$, with the probability that a stripe has not split up to a time $t$

$$
P\left(\text { splitting at } t \geqslant t_{i}^{s}\right)=P_{i}^{s}=1-(i-1) / N^{s}, i=1, \ldots, N^{s} \text {. }
$$

As shown in Fig. 3.4 (a), the probability distributions of the splitting have exponential tails (excluding the initial formation period $t_{0}$ ). This shows that stripe splitting is a memoryless process and we can therefore determine the mean time $\tau^{\mathcal{S}}(R e)$ for a splitting to occur by the following exponential ansatz:

$$
P^{s}(t)=\exp \left[-\left(t-t_{0}\right) / \tau^{s}(R e)\right]
$$

with $\tau^{s}$ estimated by the sample mean

$$
\tau^{s}=\frac{1}{N^{s}} \sum_{i=1}^{N^{s}} t_{i}^{s}-t_{0} .
$$

The sample mean is effectively the maximum likelihood estimator of the scale parameter $\tau^{s}$ [108]. $t_{0}$ has been determined in the same way as described in [18]. From Fig. 3.4(a), we find that the mean splitting time (slope of the distributions) decreases quickly with increasing Re. The exact dependence of $\tau^{s}$ on Re is shown in Fig. 3.5 
(dark square points). It turns out that the best fit for the simulation data is a superexponential function represented by the dark dashed line. Hence $\tau^{s}(\operatorname{Re})$ only approaches infinity asymptotically as Re decreases and consequently a nonzero splitting probability remains even for small Re.
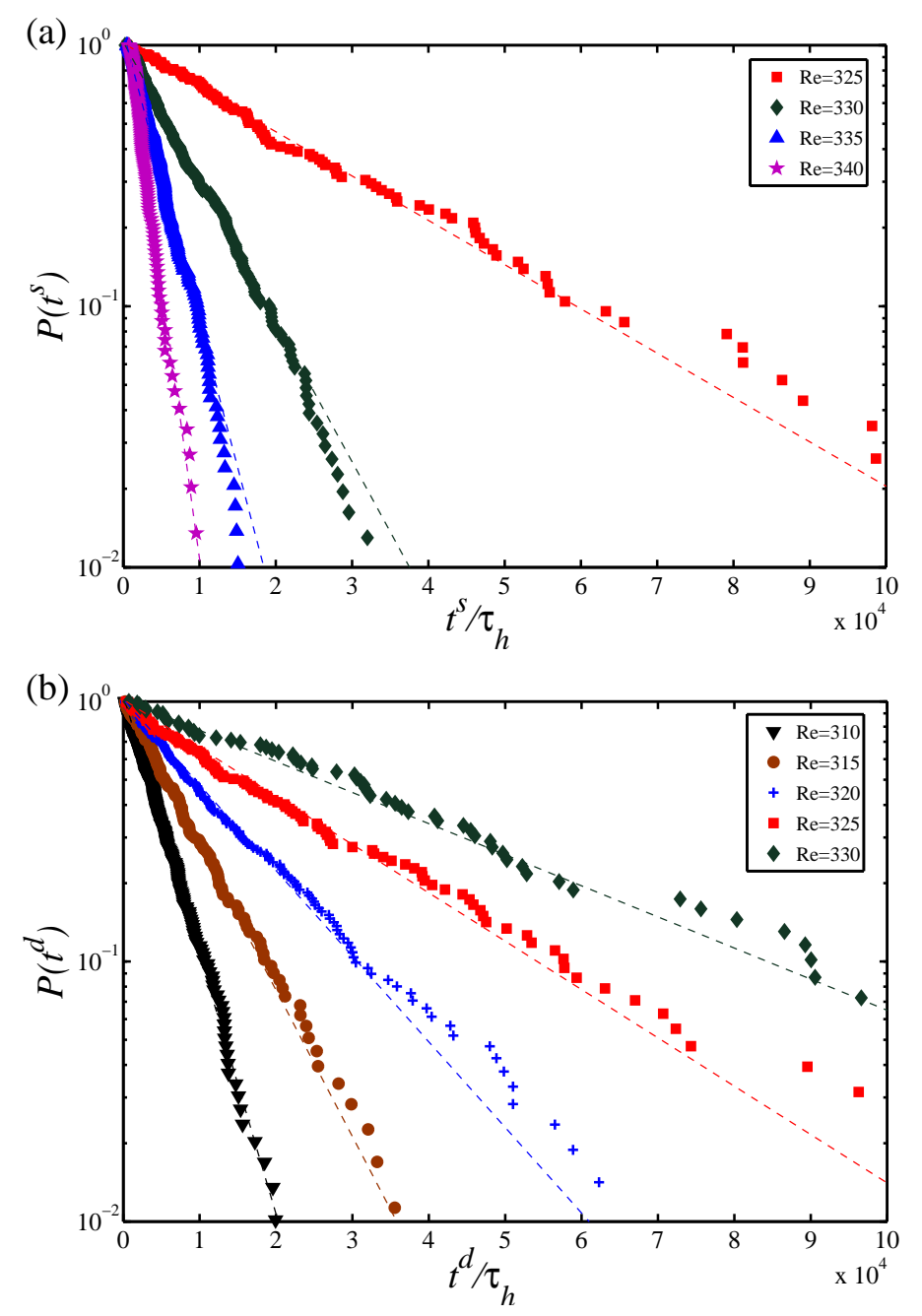

Figure 3.4: Probability distributions of a single turbulent stripe at different Reynolds numbers. (a) Splitting times; (b) lifetimes, normalized by $\tau_{h}=h / U$. The dashed lines are the corresponding exponential curves from Eq. (3.2). Y axis is in the logarithmic scale.

The same method is applied to obtain the probability distributions of the decay events (or lifetimes), the result of which is plotted in Fig. 3.4(b). The lifetimes of localized turbulent stripes are also exponentially distributed, with the mean lifetime $\tau^{d}$ scaling super-exponentially with Re (red circles in Fig. 3.5). Overall splitting and decay statistics show the same qualitative behavior as pipe flow (see Ref. [12]), suggesting that the key physical processes are largely independent of the geometry and possibly apply to many canonical shear flows. 


\subsection{Threshold of sustained turbulence}

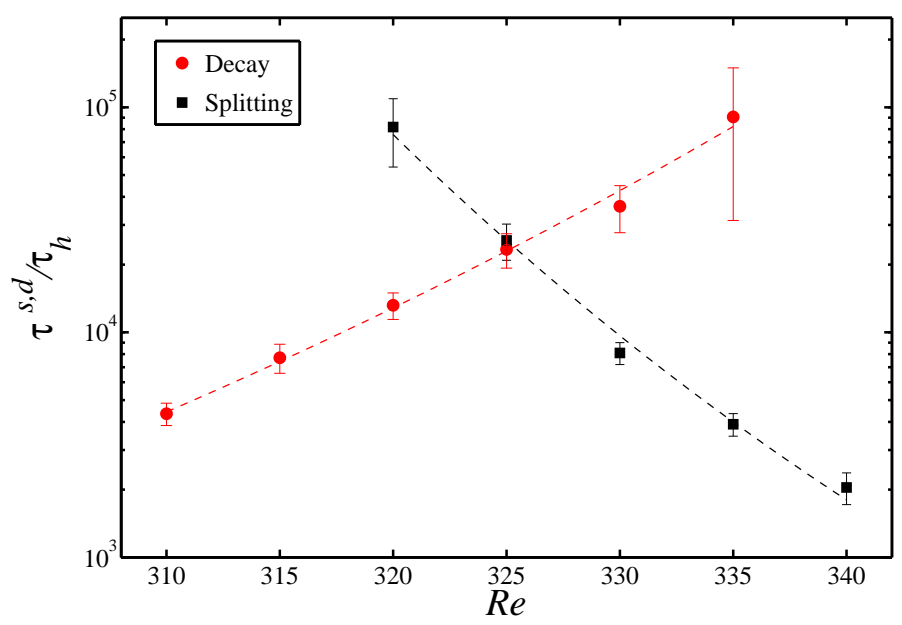

FIGURE 3.5: Scaling of the parameter $\tau^{s, d}$ with Re of the splitting (dark square) and decaying (red circle) of individual turbulent stripes. The error bars correspond to the $95 \%$ confidence intervals. The dashed lines are the super-exponential curves to guide the eyes.

The intersection of the two curves $\tau^{s, d}(\mathrm{Re})$ in Fig. 3.5 fixes a distinct Reynolds number $R e \simeq 325$ where the mean time scales of both processes are in balance, namely, $\tau^{s}(\operatorname{Re})=\tau^{d}(\operatorname{Re})$. This value gives a lower bound for the critical $\operatorname{Re}_{c}$ below which turbulence decays after a sufficiently long time and it is also very close to earlier estimates of the critical point [39, 43], although those were carried out in domains with a much larger spanwise length. While characterization of the spreading and decay processes for flows in streamwise and spanwise long domains would require excessive computation time, the same line of argument would be applicable here. Again turbulence should become sustained once growth processes (streamwise and spanwise) outweigh the decay.

\subsection{Scale invariance at criticality}

In analogy to contact processes like DP the turbulent and laminar domains can be viewed respectively as the active and passive state. In order for turbulence to survive in the system the splitting rate has to be larger than the decay rate, otherwise turbulence dies out. The onset of sustained turbulence may, in analogy to DP-like systems, become sustained at a nonequilibrium phase transition [26, 59]. If that were the case the spacing between active sites should become scale invariant close to the critical point and hence the passive regions would have no characteristic length. 


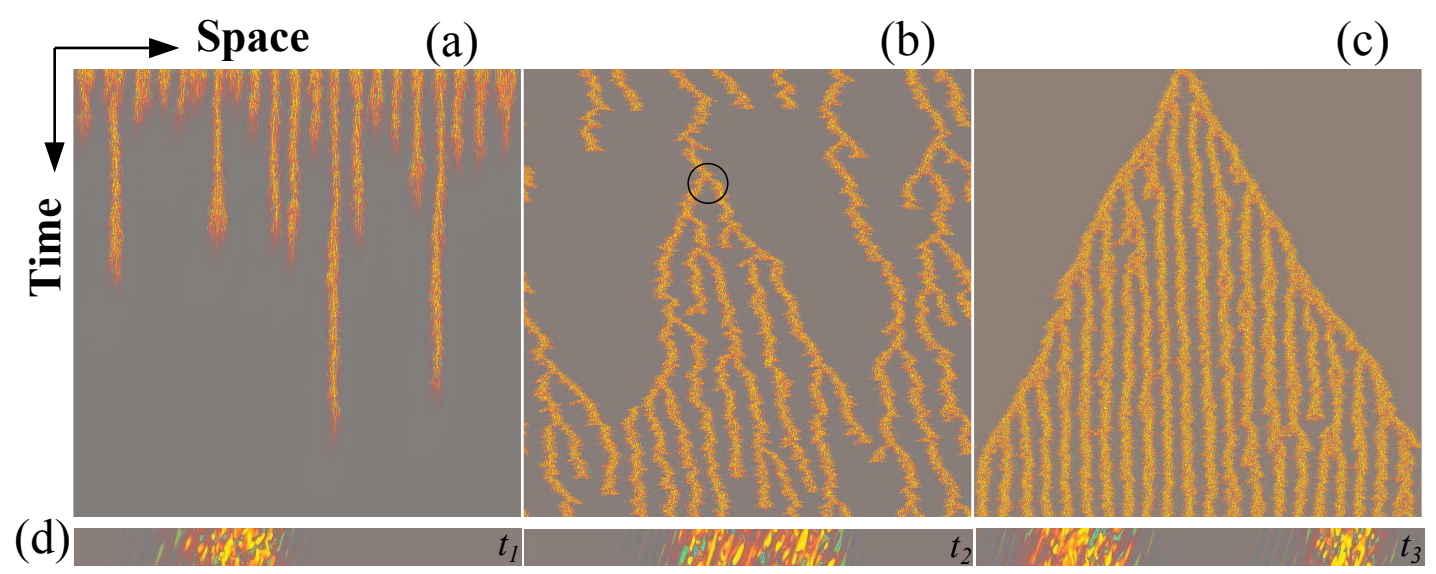

Figure 3.6: Spatio-temporal diagrams at different regimes: (a) subcritical at $\mathrm{Re}=$ 300; (b) slightly above the critical point at $R e=329.5$; (c) supercritical at $\operatorname{Re}=360$. (d) A close-up view of the splitting event at the moment indicated by the circle in (b). Snapshots are taken at the mid $\theta-z$ plane. Color map shows the streamwise vorticity.

To test this, another set of simulations are performed and we extend the box size in the stripe-normal direction $\hat{e}_{z}$ to $960 h$ such that spatial correlations are taken into account. Through the simulations at three different Reynolds numbers (subcritical, critical and supercritical), the observed dynamics agree qualitatively with the DP model (see Fig. 3.6): At $\operatorname{Re}=300$, the flow returns to the laminar state after sufficiently long time; at $\mathrm{Re}=360$, an initial single stripe quickly splits until it reaches a statistically stationary state with a typical stripe spacing. At $\operatorname{Re}=329.5$ on the other hand, turbulence persists but the stripe spacings change throughout, exploring all scales permitted in the given domain size. An example of stripe splitting is shown in Fig. 3.6(d). After an initial increase in width, the stripe breaks up into two segments of similar size that then continue to separate. It should be noted that this process differs from puff splitting in pipes where the new puff originates from a thin filament of vorticity that disconnects from the original puff (Fig. 2A in [12]). Furthermore, in pipes, splitting exclusively occurs in the downstream direction whereas in the present case no preferable splitting direction exists.

For a quantitative evaluation of the laminar spacing, sizes of laminar gaps are measured by setting a cutoff value to the averaged vorticity, below which the flow is considered to be laminar, and the distributions are tested to be insensitive to the cutoff value (except for shifts of the absolute values). Data are sampled at the (quasi-)stationary state, over a time interval of $\mathcal{O}\left(10^{5}\right) \tau_{h}$. It is observed that the size distributions sufficiently above critical are exponential and hence possess a characteristic size. Close to the critical point the distributions follow a power law instead ( $\mathrm{Re}=329$ in Fig. (3.7), implying that there is no characteristic length. Size distributions hence indeed exhibit scale invariance, confirming that the intersection point in Fig. 3.5 marks a phase transition and the onset of sustained turbulence. Furthermore the circumstance that 
size distributions follow a power law close to criticality and become exponential at larger supercritical values shows that the resulting intermittent flows are intrinsically irregular and do not form fixed patterns as had been proposed previously [47, 109].

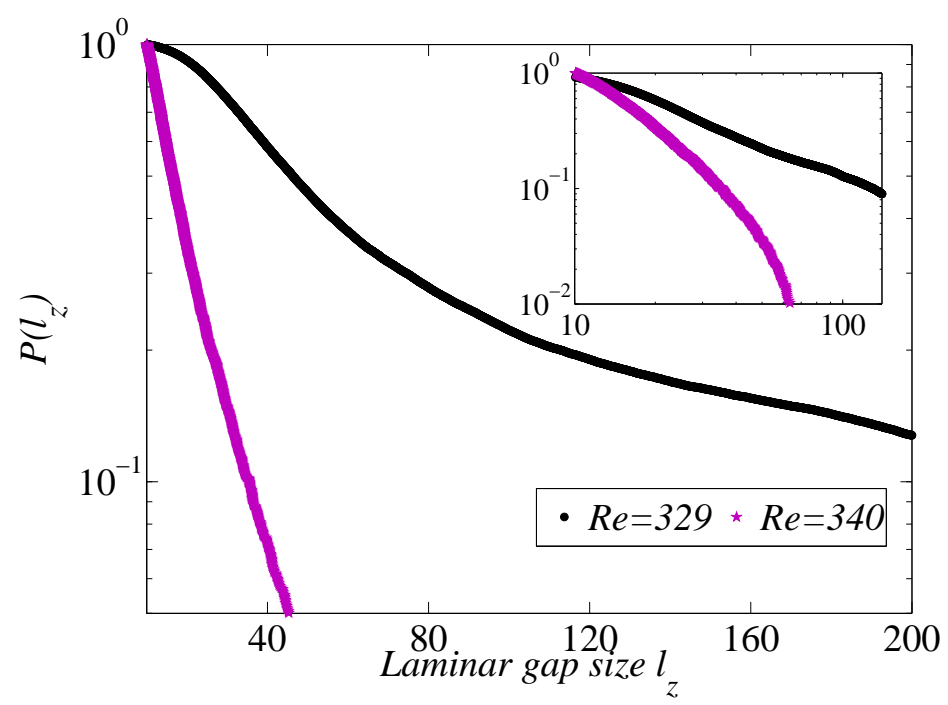

Figure 3.7: Size distributions of laminar gaps at $\mathrm{Re}=329$ and $\mathrm{Re}=340$ in semilogarithmic scale. The length in $z$ direction is $L_{z}=960 h$. Inset is the plot in log-log scale.

\subsection{Critical exponents at criticality}

While the feature of scale invariance observed above shows that the transition to turbulence is a nonequilibrium continuous phase transition, measurement of the critical exponents at criticality allows us to test Pomeau's conjecture [11], on whether the subcritical transition to turbulence belongs to the DP universality class. Simulations with different initial conditions have been performed at $R e \in[328,360]$. The spatial length $L_{z}$ is required to be long enough to avoid the finite-size effects. However, to make a better trade-off between the spatial extension and the computational costs, the size of the computational domain in our simulations depends on Re: $L_{z}$ is larger at Re closer to the critical point while the other two directions are kept unchanged. Table 3.1 lists the Re-dependent domain sizes and the corresponding resolutions. At each Re, five runs with different initial conditions have been done over a time inteval of $\mathcal{O}\left(10^{4}-10^{5}\right) \tau_{h}$. The laminar-gap size and the turbulent-stripe length in $Z$ direction are obtained with the same method as described in Sec. 3.5.

The time series of the ensemble-averaged (averaged over all realizations at each Re) turbulent fraction $F_{T}(t)$ is shown in Fig. 3.8. As Re approaches the critical point, the fluctuations of the ensemble-averaged turbulent fraction become larger and this 
Chapter 3. Onset of sustained turbulence in Couette flow

\begin{tabular}{cccc}
\hline \hline $\operatorname{Re}$ & 328.85 & {$[329,332.5]$} & {$[335,360]$} \\
\hline$L_{z}$ & $1920 h$ & $960 h$ & $480 h$ \\
$n_{z}$ & 12288 & 6144 & 3072 \\
\hline \hline
\end{tabular}

TABLE 3.1: The computational domain size $L_{z}$ and the corresponding resolutions for different Re. The other two directions have a size of $\left(L_{\theta} \times L_{r}\right)=(10 h \times 2 h)$ with resolution of $(48 \times 32)$.

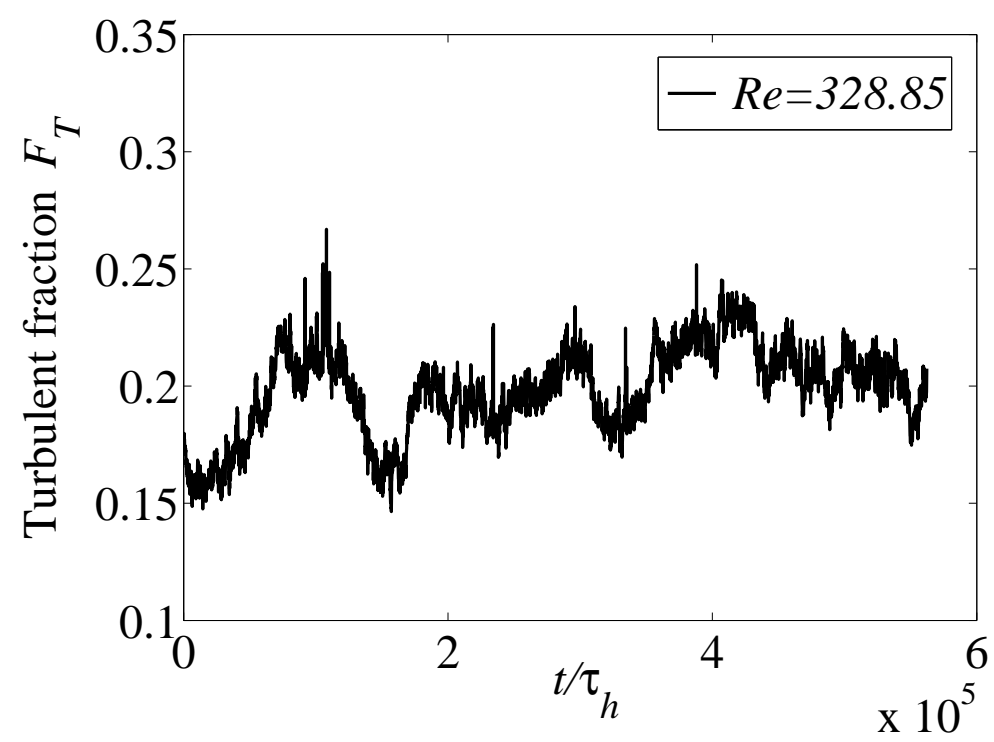

FiguRE 3.8: Temporal evolution of the ensemble-averaged turbulent fraction $F_{T}$ at $R e=328.85$. Time is in the advective time unit $\tau_{h}=h / U$.

requires longer time to obtain better statistics. At the statistically steady state, we take the temporal mean of the ensemble-averaged turbulent fraction $F_{T}(t)$, labeled here "mean turbulent fraction" $\bar{F}_{T}$. The mean turbulent fraction depends solely on Re and is considered as the order parameter in our system. Below the critical Reynolds number $R e^{c}, \bar{F}_{T}=0$, while $\bar{F}_{T}>0$ for $R e>R e^{c}$, as shown in Fig. 3.6.

The mean turbulent fraction $\bar{F}_{T}$ is plotted against Re in Fig. 3.9 and is then fitted with different power-law functions $\left(\bar{F}_{T} \sim \epsilon^{\beta}\right.$ where $\left.\epsilon=\frac{R e-R e^{c}}{R e^{c}}\right)$ as listed in Table 3.2 . In Fig. 3.9, the black square points are obtained from simulations. The red dashed line shows the power-law fit with a fixed exponent $\beta=0.276$, which is here called " $\beta$-fit ". The $\beta$-fit is done for Re $\in[328.85,332.5]$, giving a critical Reynolds number $R e_{c}^{\beta \text {-fit }} \simeq 328.65$. Note that the chosen exponent is the universal value in the onedimensional DP universality class (see Table 1.1). Within the error bars and the range of fitting, the numerical date matches very well with the fitting line. In the best fit (the blue dot-dashed line), the exponent $\beta$ is a fitting parameter, together with another two: the coefficient $a$ and the critical value $R e_{c}$. The power exponent from the best fit is $\beta^{\text {best-fit }} \simeq 0.211$, about $24 \%$ lower than the $1 \mathrm{D}$-DP value. The critical Reynolds 
number is almost the same $R e_{c}^{\text {best-fit }} \simeq 328.76$, although the fitting range of Re is different.

\begin{tabular}{cccccc}
\hline \hline power fit & fitting function & Re range & $a$ & $R e_{c}$ & $\beta$ \\
\hline$\beta$-fit & $a\left(R e-R e_{c}\right)^{0.276}$ & {$[328.85,332.5]$} & 0.323 & 328.65 & 0.276 \\
best fit & $a\left(R e-R e_{c}\right)^{\beta}$ & {$[328.85,340]$} & 0.337 & 328.76 & 0.211 \\
\hline \hline
\end{tabular}

TABLE 3.2: The power-law fits of the mean turbulent fractions: $\beta$-fit has two fitting parameters $\left(a, R e_{c}\right)$ with a fixed $\beta=0.276$, the $1 \mathrm{D}-\mathrm{DP}$ universal value; In the best fit, $\beta$ is an additional fitting parameter.

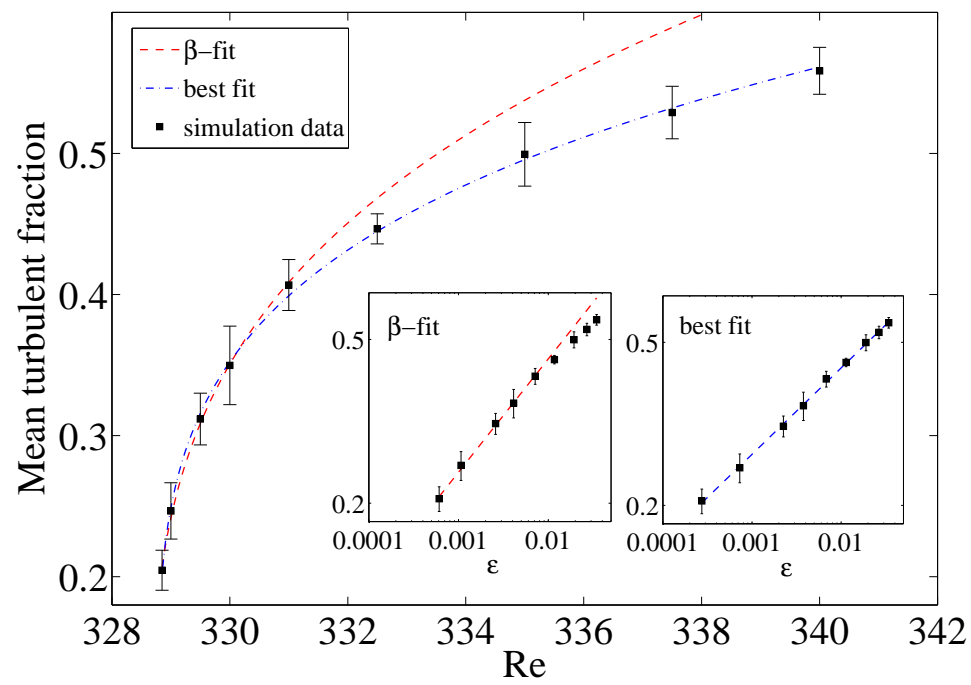

FiguRE 3.9: Mean turbulent fraction $\bar{F}_{T}$ as a function of Re. The black square points denote the numerical data. The red dashed line is the power fit with fixed exponent $\beta=0.276$ (" $\beta$-fit") while the blue dot-dashed line is the best power fit. The fits give a critical Reynold number $R e_{c}=328.5 \pm 0.5$. Error bars correspond to the standard deviation. Inset is log-log plots, with the normalized Reynolds number $\epsilon=\frac{R e-R e^{c}}{R e^{c}}$ in the $X$ axis.

Different fitting methods have also been tried. One example is the best fit within various range of Re (number of data points) (see Table 3.3). For different fits, the critical value $R e_{c}$ changes very little, only at the first or even the second decimal place. Hence, $R e_{c}=328.5 \pm 0.5$. Note that the critical Reynolds number is slightly higher than the crossing point $R e \simeq 325$ in Fig. 3.5. In statistical phase transitions, the real critical point typically occurs not at the balance point of two competing processes, but at a higher point where the spreading rate slightly outweighs the decay one [12].

However, the exponent $\beta$ from different fits varies approximately from 0.21 to 0.28 , very sensitive to the data point closest to criticality and the fitting method. Certainly, more statistics are required to obtain a conclusive value for $\beta$. Besides, a more reliable way to obtain $\beta$ is to fit the data with decreasing number of fitting points until the fitting $\beta$ converges to a certain value. The reason is that in the 1D-DP universality class the scaling $\bar{F}_{T} \sim \epsilon^{0.276}$ holds only at criticality. Therefore, the deviation of the above 
$\beta^{\text {best-fit }}$ from the universal value does not exclude the possibility that the transition falls into the DP universality class. Moreover, the agreement between the $\beta$-fit and the simulation data near the criticality are actually very good.

\begin{tabular}{cccccc}
\hline \hline \# points & 5 & 6 & 7 & 8 & 9 \\
\hline$\beta$ & 0.247 & 0.229 & 0.223 & 0.216 & 0.211 \\
$R e_{c}$ & 328.71 & 328.74 & 328.75 & 328.76 & 328.76 \\
$a$ & 0.330 & 0.332 & 0.333 & 0.335 & 0.335 \\
\hline \hline
\end{tabular}

TABLE 3.3: The best fits of the mean turbulent fractions with different number of data points. "5" means the first 5 points in Fig. 3.9.

Furthermore, we analysed the probability density function (PDF) of laminar gap sizes at criticality. As shown in Fig. 3.10, the PDF at Re close to the critical point follows a power law, whereas at $R e=335$ it deviates obviously from a power-law scaling, which further confirms the feature of scale invariance at criticality as illustrated in 93.5. At $R e=329$, the power exponent from the best fit is $\mu \simeq-1.75$. According to [51], at criticality this so-called empty-interval exponent is linked to the spatial critical exponent $\nu_{\perp}$ by the following relation, $\mu_{\perp}=\beta / \nu_{\perp}-2$. Therefore, we have $\left.\nu\right|_{R e=329} \simeq 1.1$, which is very close to the universal value 1.097 and hence provides evidence that the transition in our system belongs to 1D DP universality class.

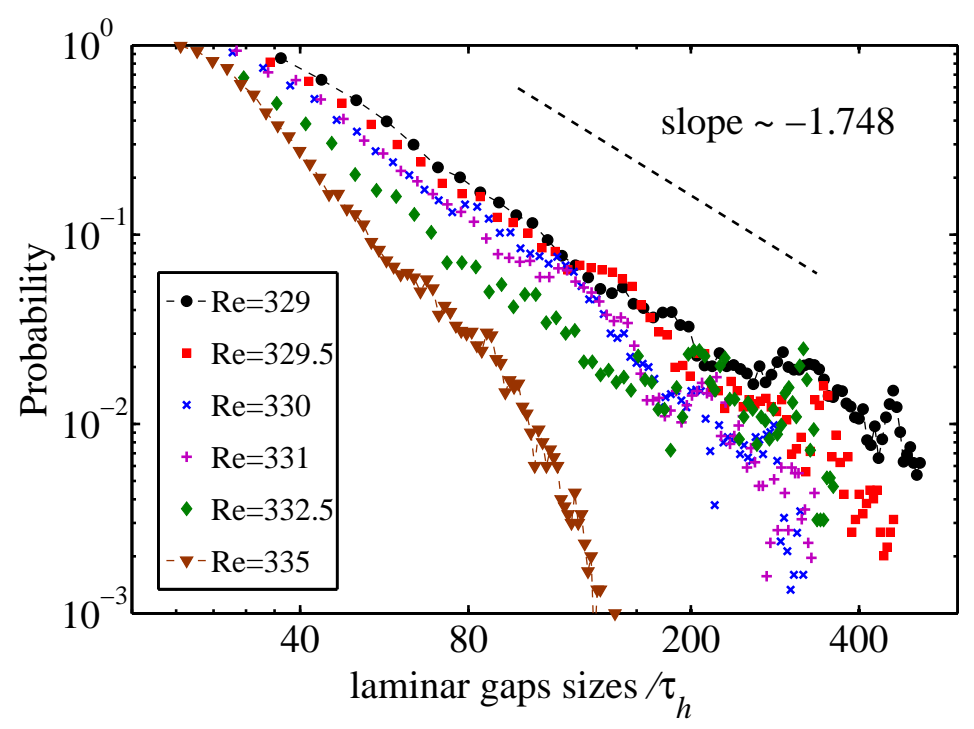

Figure 3.10: Probability density function (PDF) of laminar gap sizes at different Re. The line with slope -1.748 (the value of 1D DP class) serves as guide to our eye. Both axes are in logarithmic scale.

In contrast, the width of turbulent stripes are exponentially distributed even at Re close to the critical point, meaning that there is a characteristic length for the turbulent stripe. Figure 3.11 shows the cumulative probability distributions of the widths of turbulent stripes. All curves collapse together, with a mean width of about $41 h$ (see 
Fig. (3.12). This value is, to the best of my knowledge, for the first time quantitatively obtained and is consistent with the observations from previous experiments [47] and numerical simulations [48]. It is worth to note that this value is slightly dependent on the cutoff vorticity level below which the flow is considered to be laminar.

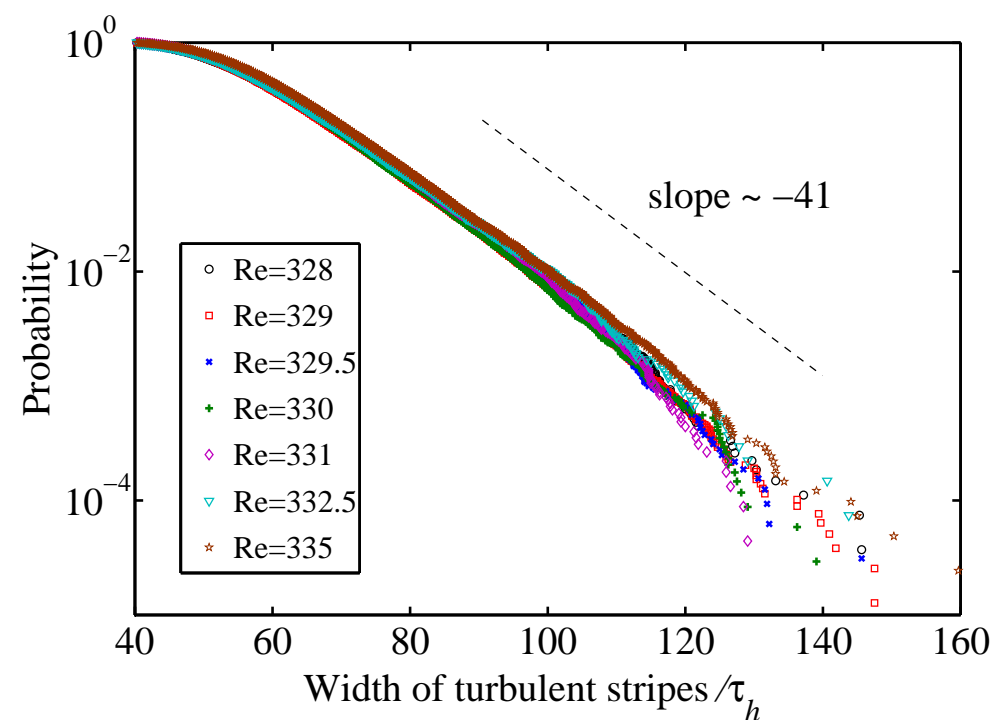

Figure 3.11: Probability distributions of the widths of turbulent stripes at different Re. $Y$ axis is in logarithmic scale. The slope " 41 " is the slope of the exponential functions in log-scale, meaning the characteristic length of turbulent stripes.

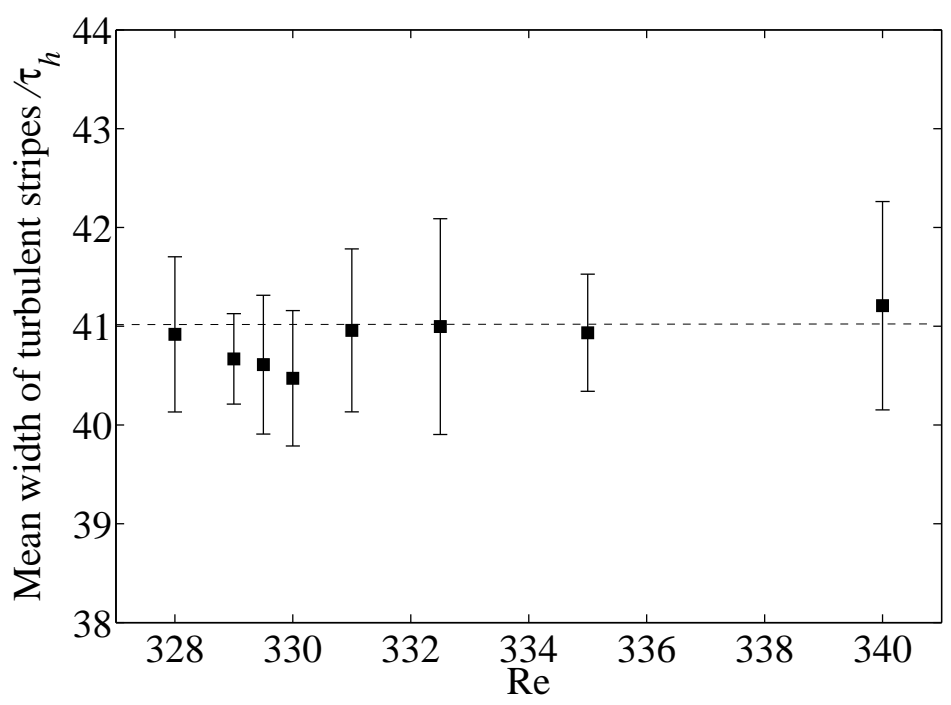

Figure 3.12: Mean turbulent width as a function of Re. The error bars indicate the $95 \%$ confidential interval. 


\subsection{Discussion and summary}

Laminar-turbulent intermittency is intrinsic to the transitional regime of a wide range of fluid flows including pipe, channel, boundary layer, and Couette flow. In the latter turbulent spots can grow and form continuous stripes, yet in the stripe-normal direction they remain interspersed by laminar fluid. We carried out direct numerical simulations in a long narrow domain and observed that individual turbulent stripes are transient. In agreement with recent observations in pipe flow, we find that turbulence becomes sustained at a distinct critical point once the spatial proliferation outweighs the inherent decaying process. Furthermore, by resolving the asymptotic size distributions close to criticality we demonstrate scale invariance at the onset of turbulence, which indicates that the transition to turbulence is a continuous (2nd order) phase transition. The power-law exponents associated with the turbulent fraction and the laminar gap sizes are in reasonably good agreement with the universal values in the 1D-DP class and hence support that the transition to turbulence in Couette flow belongs to the DP universality class.

These results are robust in the "tilted" slender computational box of this study. The flow is three dimensional but its dynamics is quasi-1D, mainly constrained in the direction perpendicular to the stripe ( $Z$ direction in Fig. 3.2 (right)). Although our flow may not be a general PCF system and it is certainly very tricky to check our results in experiments, it is not a model or a simple system of ODE. It represents a real flow, the motion of which governed by the Navier-Stokes equations. To extend our results to the general PCF, one may still need to consider the following factors:

- The domain size. While the size in $Z$ direction is spatially extended, the size in $\theta$ direction is relatively short. This choice actually turns out to be a very successful strategy to simplify the complicated dynamics and to make the whole study possible within our current computing resources. However, the length $L_{\theta}$ has an influence on the results. As is shown in our previous study [106], doubling the size in $\theta$ direction (from $10 h$ to $20 h$ ) steepens the exponential distributions of the splitting time. This indicates that a longer turbulent stripe tends to split at an earlier moment, which may saturate as we further increase $L_{\theta}$. Whether it would influence the critical Reynolds number is unknown and worth further investigation.

- The "titled" angle. In all our simulations, this angle is fixed to be $24^{\circ}$. In a general PCF, this angle varies a lot, ranging from $20^{\circ}-70^{\circ}$ [43]. However, we believe that this angle will not change the results in a titled box.

- Simulation in the conventional PCF domain (as in Fig. 3.2, left). In this box, the dynamics is two dimensional. Besides the decay and splitting events, the 
stripe can also break into spots as Re decreases and grow along the stripe as Re increases. To quantify the dynamics in this case, the measurements of turbulent fraction and splitting time are difficulties. Especially close to the critical point, turbulent spots dominate the dynamics and it is thus hard to define a splitting event. One may define splitting as the doubling of the turbulent fraction. Since simulation would be too costly, experiments may be a better choice.

The last remark is on the temporal exponent. In our quasi-1D system, the calculation of the temporal exponent is very difficult. Theoretically, both steady-state and quenching experiments ${ }^{1}$ can be employed to calculate the temporal exponent [51]. In steady-state experiments, one can obtain the data of the time empty intevals from the space-time diagrams and then calculate the probability distributions, as is done for the turbulent liquid crystals in [55]. However, the mean decay or splitting time in Couette flow is about $2 \times 10^{4} \tau_{h}$ at criticality while the longest running time $\left(\sim 5 \times 10^{5} \tau_{h}\right)$ is still too short to obtain sufficient statistics for the temporal exponent. On the other hand, in quenching experiments 5 runs at each Re result in big fluctuactions even at the initial relaxation stage. Moreover, the computation domain is still not big enough to reach very small turbulent fraction. However, by performing the under-resolved simulations, we are able to have 20 runs at each Re and indeed we observe collapse of time series of the turbulent fraction by rescaling the axes (see Appendix B). In summary, the calculation of the temporal exponent requires much larger realizations and much longer integration time, setting a challenge for future studies.

\footnotetext{
${ }^{1}$ In steady-state experiments, data are sampled and results are obtained at the statistically steady states. However, quenching experiments depart from a fully turbulent state but run at Re close to the critical point, mainly devoted to study the dynamical behaviors before the flow decays or before it reaches the steady states.
} 
"THE "PARADOX" IS ONLY A CONFLICT BETWEEN REALITY AND YOUR FEELING OF WHAT REALITY "OUGHT TO" BE. “

Richard P. FEynman (1918 - 1988)

\begin{abstract}
4
Nonlinear transition to TURBUlEnCE IN QUASI-KEPLERIAN FLOW
\end{abstract}

Astrophysical disk flows must be turbulent so that the angular momentum can be transported outwards in the disk, as the matter spirals inwards and accretes onto the central massive body (see Fig 1.5). However, the origin of turbulence is still unclear. The Keplerian angular velocity profile, decreasing radially as $\Omega(r) \sim r^{-3 / 2}$, is linearly stable according to the inviscid Rayleigh criterion [2] and hence alternative mechanisms are required for turbulence to arise. In hot and ionized disks the magnetorational instability gives rise to turbulence [68 70], but it nonetheless fails to operate in cold and poorly ionized disks. However, as shown in Chapter 93 , in PCF and other linearly stable flows subcritical transition to turbulence via nonlinear mechanisms may apply to accretion disk flows. This Chapter is devoted to check whether subcritical turbulence arises in Rayleigh stable rotating flows. This question has been recently studied in the quasi-Keplerian regime (Regime II in Fig. 1.2) in TCF. The experimental results from different groups yielded contradictory results. More background can be found in $\$ 1.4$. This discrepancy may arise from the axial boundary conditions: numerical simulations of the experimental setups show that top and bottom end walls confining the fluid strongly disrupt Keplerian velocity profiles and causes turbulence to arise already at $R e \sim \mathcal{O}\left(10^{3}\right)$ [75]. Hence, the interpretation and extrapolation of experimental data remains controversial because of the prominent role played by axial end walls. To avoid end-wall, we here perform direct numerical simulations of TCF with axially periodic 
boundary conditions. At shear Re as large as $1 \times 10^{5}$ no sustained turbulence has been found. Although the flow is strongly disturbed and develops quickly into a turbulent state, the disturbance eventually decay but in very long time duration.

\subsection{Numerical specification}

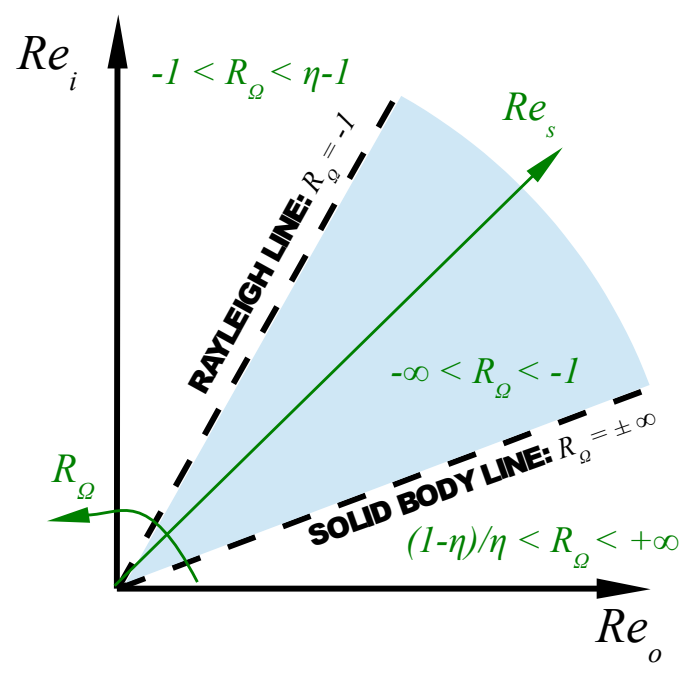

Figure 4.1: The parameter space $R e_{s}-R_{\Omega} . R_{\Omega}$ is constant along the half-line starting from origin. The range of $R_{\Omega}$ is shown at different regions seperated by the Rayleigh line $\left(R_{\Omega}=-1\right)$ and the solid body line $\left(R_{\Omega}= \pm \infty\right)$.

Following the parameter choice of Dubrulle et al. [110], we introduce two dimensionless parameters, namely, the shear Reynolds number $R e_{s}$ and the rotation number $R_{\Omega}$ :

$$
R e_{s}=\frac{2}{1+\eta}\left|\eta R e_{o}-R e_{i}\right|, \quad R_{\Omega}=\frac{(1-\eta)\left(R e_{i}+R e_{o}\right)}{\eta R e_{o}-R e_{i}}
$$

The shear Reynolds number $R e_{s}$ characterizes the shear between the inner and outer cylinders while the rotation number $R_{\Omega}$ is a measure for the mean rotation and is constant on every half-line out from the origin in the $R e_{o}-R e_{i}$ space (see Fig. 4.1). On the solid-body line, there is no relative motions between different layers and hence $R e_{s}=0$, whereas $R_{\Omega}= \pm \infty$. For comparison to the recent experimental and numerical results, we choose the radius ratio $\eta=0.71$. This value is fixed for all the simulations. The simulations have been performed at three different Reynolds numbers $R e_{i}=\left[1 \times 10^{4}, 1 \times 10^{5}, 2 \times 10^{5}\right]$ on the half line $R_{\Omega}=-1.2$. The corresponding shear Reynolds number is $R e_{s}=[5078.8,50788,101576]$. Another relevant parameter often encountered in the literature is the local exponent of angular velocity $q=-d \ln \Omega / d \ln r$. For a Keplerian velocity profile, $q=3 / 2$, and on the Rayleigh line $q=2$. Note that for the TCF base flow the parameter $q$ is not constant in the radial direction. In all our 
simulations, $q(r)=\frac{2}{r^{2}+\text { constant }} \in[1.5,1.8]$, which is between the Keplerian line and the Rayleigh line in the $R e_{o}-R e_{i}$ space (the blue region in Fig. 4.1). A comparison between astrophysical Keplerian flow and TCF of our simulations is shown in table 4.1.

\begin{tabular}{cccccc}
\hline \hline & $\Omega^{b}(r)$ & $R e_{s}$ & $R_{\Omega}$ & $q(r)$ & axial boundary \\
\hline our TCF simulations & $C_{1} r+C_{2} / r$ & $10^{4-5}$ & -1.2 & {$[1.5,1.8]$} & periodic \\
Keplerian & $C r^{-3 / 2}$ & $\gg 10^{6}$ & $-4 / 3$ & $3 / 2$ & free surfaces \\
\hline \hline
\end{tabular}

TABLE 4.1: A parameter comparison between TCF of our study and astrophysical Keplerian flows: base angular velocity profile $\Omega^{b}(r)$, shear Reynolds number $R e_{s}$, rotation number $R_{\Omega}$, local exponent $q(r)$ and axial boundary conditions. $C_{1}$ and $C_{2}$ are defined in Eq. 1.1 while $C$ is a constant.

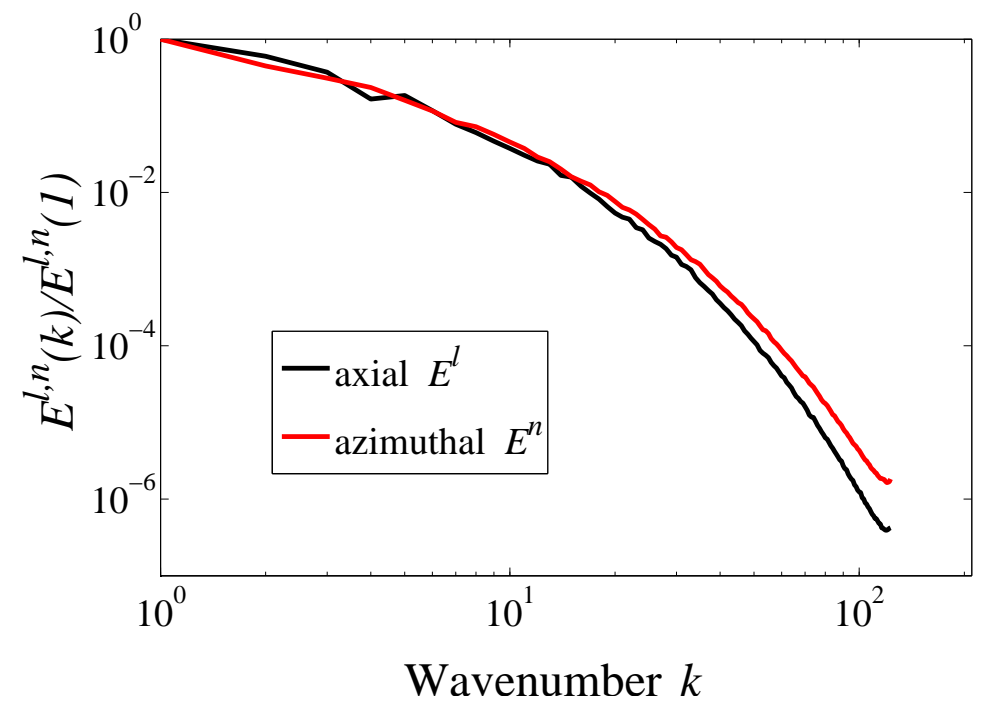

FIGURE 4.2: The axial (black) and azimuthal (red) energy power spectra $E^{l, n}(k)$ at time $t=4 \times 10^{-4} \tau_{\nu}$, before the decay of turbulence. The wavenumber $k$ corresponds to the axial or azimuthal wavenumber.

We use the parallel code nsCouette to simulate the Taylor-Couette flow. The technical details and the accuracy of the code are presented in section 92.3 The corresponding parameters of the simulations are listed in table 4.2. As for the computational domain, we choose $1 / 8$ cylinder in the azimuthal direction and $\Gamma=L_{z} / d=2$ at $R e_{s}=5078.8$. The total number of grid points $\left(N_{r} \times N_{\theta} \times N_{z}\right)$ is $(256 \times 256 \times 256)$. This resolution is checked by the axial and azimuthal energy spectra (see Fig. 4.2). To save computing time, the domain size for higher Reynolds numbers is chosen to be smaller, 1/16 cylinder in $\theta$ direction and $\Gamma=0.5$. As we will shown in the next section, this domain is sufficient to hold the building blocks (streaks and vorticities) of turbulence. At higher Re the spatial resolution in each direction is proportionally increased as $N \sim R e^{3 / 4}$, given that the domain size is the same. We should point out that in the case II a lower resolution than the one shown in Table 4.2 causes the simulations to blow up. This may be explained by the fact that with a lower resolution the smallest scale where the energy dissipates is not resolved such that the energy accumulates in the flow and 
causes the simulations to explode. The time step in Table 4.2 is the maximal value which allows the simulations not to blow up.

\begin{tabular}{ccccccc}
\hline \hline No. & $R e_{i}$ & $R e_{s}$ & $k_{\theta}$ & $\Gamma$ & \# points & $\mathrm{dt}$ \\
\hline I & $1 \times 10^{4}$ & 5078.8 & 8 & 2 & $256 \times 256 \times 256$ & $10^{-9}$ \\
II & $1 \times 10^{5}$ & 50788 & 16 & 0.5 & $1152 \times 384 \times 384$ & $10^{-9}$ \\
III & $2 \times 10^{5}$ & 101576 & 16 & 0.5 & $2048 \times 768 \times 512$ & $5 \times 10^{-10}$ \\
\hline \hline
\end{tabular}

TABLE 4.2: DNS parameters of TCF in quasi-Keplerian regime. The radius ratio is $\eta=0.71$. Periodic boundary conditions are imposed in axial direction.

Our initial conditions are derived from the optimal perturbations from a study of transient growth by Maretzke et al. [104], on top of which small three dimensional noise is added. Since the global optimal azimuthal wavenumber $k_{\theta}^{\text {opt }}$ for the transient growth is 7 (Table 4.3), smaller than $k_{\theta}$ used in our simulations, only part of the optimal perturbations are used as the initial conditions. For the case $R_{\Omega}=-1.2$ and $R e_{s}>\mathcal{O}\left(10^{3}\right)$, the optimal axial wavenumber equals to zero, indicating an axiallyindependent structure. The optimal transient growth rate $G^{\text {opt }}$ at these Reynolds numbers are listed in table 4.3. All simulations have been run on a Intel cluster (HYDRA in Garching Computing Center RZG) and over a time duration $\sim \mathcal{O}\left(10^{2}\right) \frac{d}{U}$ (about 13 cylinder rotations) in our best effort. The simulations are very costly in computing time: the simulation III takes about $5 \times 10^{6}$ core hours, with 512 MPI tasks and 10 OpenMP threads. The results are shown and discussed in the remaining of this Chapter.

\begin{tabular}{cccccc}
\hline \hline No. & $R e_{i}$ & $G^{\text {opt }}$ & $t^{\text {opt }} / \tau_{\nu}$ & $k_{z}^{\text {opt }}$ & $k_{\theta}^{\text {opt }}$ \\
\hline I & $1 \times 10^{4}$ & 15.00 & 0.00185 & 0 & 7 \\
II & $1 \times 10^{5}$ & 75.15 & 0.00040 & 0 & 7 \\
III & $2 \times 10^{5}$ & 120.19 & 0.00025 & 0 & 7 \\
\hline \hline
\end{tabular}

TABLE 4.3: The transient growth rate of the initial perturbations attained at time $t^{\text {opt }}$ and the corresponding optimal wavenumber.

\subsection{Numerical results}

We first measured the time series of the total perturbation energy. Assuming that $\hat{\mathbf{u}}^{l n}(r)=\hat{\mathbf{u}}\left(r, l k_{\theta}, n k_{z}\right)$ are the spectral coefficients in Fourier space of the velocity field $\mathbf{u}(r, \theta, z)$, the modal kinetic energy $E^{l n}$ associated with the Fourier mode $(l, n)$ is defined as

$$
E^{l n}=\frac{1}{2} \int_{r_{i}}^{r_{o}}\left[\hat{u}_{r}^{l n}(r)^{2}+\hat{u}_{\theta}^{l n}(r)^{2}+\hat{u}_{z}^{l n}(r)^{2}\right] r d r .
$$


We also need the kinetic energy of the axial mode $l$ and of the azimuthal mode $n$, respectively,

$$
E^{l}=\sum_{n=-N}^{N} E^{l n}, \quad E^{n}=\sum_{l=-L}^{L} E^{l n} .
$$

The total kinetic energy can be therefore expressed as

$$
E=\sum_{l=-L}^{L} \sum_{n=-N}^{N} E^{l n}
$$

Deducing the laminar energy from the total energy gives the perturbation energy $E_{p}$, the formulation of which differs only at mode $(0,0)$ in Eq. 4.2 by replacing $\hat{u}_{\theta}^{00}(r)$ with $\left[\hat{u}_{\theta}^{00}(r)-U_{\theta}^{b}(r)\right]$. Figure 4.3 shows the temporal evolution of the total perturbation energy $E_{p}(t)$ normalized by the initial perturbation energy $E_{0}$ at all Re of this study. The time is here in viscous unit $\tau_{\nu}=d^{2} / \nu$.

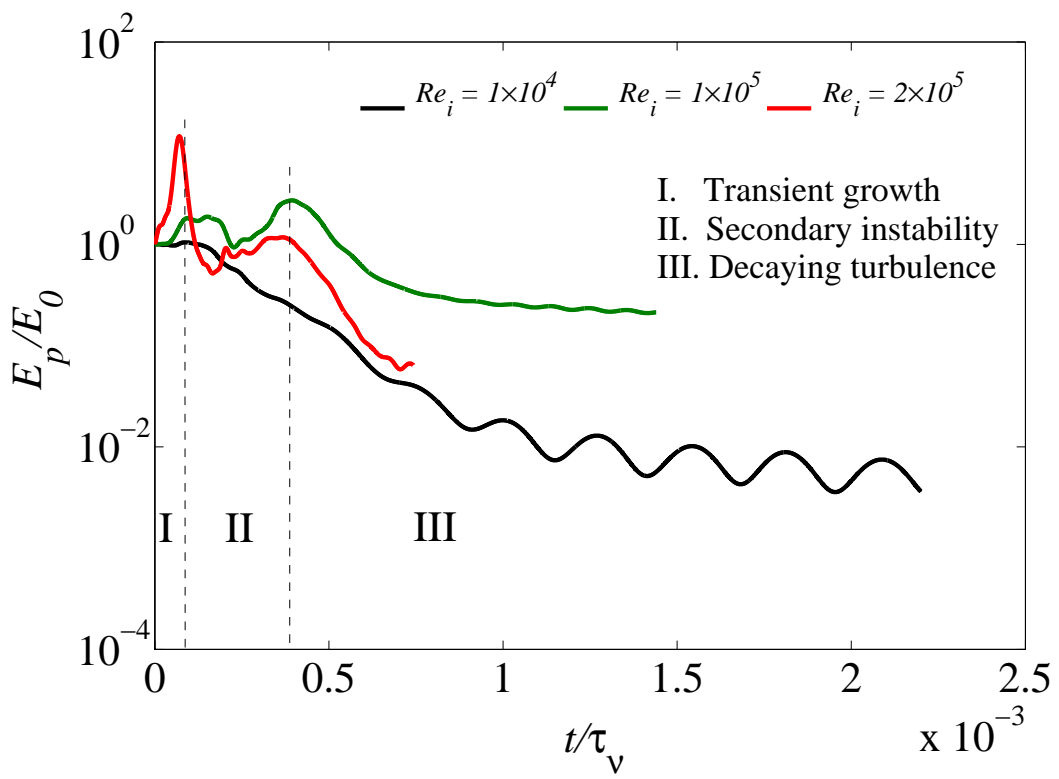

Figure 4.3: The temporal evolution of the total perturbation energy $E_{p}(t)$ normalized by the initial perturbation energy $E_{0}$. The dashed lines are guided lines seperating three different stages while different color corresponds to different $R e_{i}$. The time is in viscous unit $\tau_{\nu}=d^{2} / \nu$.

In all cases the perturbation energy decays eventually. However, the whole process occurs in a very long viscous time scale. We identify three different stages: 1) the first stage is mainly the transient growth of the initial perturbations which is attributed to the non-normality of the eigenmodes in the linearized Navier-Stokes equations. This stage happens within relatively short time since the nonlinear effects takes over once the amplitudes of the higher modes become non-negligible. 2) The nonlinear interactions give rise to secondary instabilities which will be further discussed in section \$4.2.1, 
These instabilities pump energy from the mean flow to the small scale structures and drive the flow turbulent. This is the second stage. 3) At the third stage, however, turbulence can not maintain its momentum due to the viscous and Coriolis forces and starts decaying, but with a different decay rate at different Re.

\subsubsection{Secondary instability}

Linear stability analysis tells us that the primary mode has negative growth and will eventually decay for TCF in the quasi-Keplerian regime [104]. As mentioned above, the most amplified axial mode in our case is $l=0$. The inset in Fig. 4.4 shows the nonlinear temporal evolution of the modal energy for $l=0$, which is the perturbation energy of the axially-averaged flow. The evolution follows qualitatively the prediction of the linear stability analysis: After the initial transient growth, the energy starts decaying. Note that the time for reaching the maximal point here is different from the optimal time listed in table 4.3 and that the transient growth rates are also much smaller than in the linear system. One reason is that the initial conditions in our simulations are not exactly only part of the optimal perturbations. The transient growth of the primary mode distorts the mean flow and supplies energy to the higher modes (small-scale structures). If the distortion is strong enough it causes the rapid growth of secondary modes. Figure 4.4 shows the exponential growth of the first eight axial modes at the early moment for $R e_{i}=1 \times 10^{5}$. At all Re, the secondary instabilities happen very fast, in the time interval $[1,4] \times 10^{-4} \tau_{\nu}$, and then drive the mean flow breakdown to a seemingly turbulent state. The energy power spectra $E^{l, n}(k)$ and dissipation spectra $k^{2} E^{l, n}(k)$ at time $t=4 \times 10^{-4} \tau_{\nu}$, where the turbulence intensity is nearly the strongest, is shown in Fig. 4.5. The power spectra shows that the flow is well resolved. The inertial range is short and the energy dissipation peaks at wavenumber $k<10$, meaning that turbulence here has relatively large "eddy" structures.

\subsubsection{Decaying turbulence}

The arising turbulence starts decaying at time $t \simeq 4 \times 10^{-4} \tau_{\nu}$, at all Re of this study. This can be seen from the azimuthal and axial modal energy at different Re. As shown in Fig. 4.3, after the secondary instabilities the perturbation energy firstly decays exponentially and switches finally to a wavy route downwards and with a lower decay rate. Figure 4.6 shows the temporal evolution of the velocity components at one point at mid gap at different Re. We can see that the fluctuation of $u_{z}$ fades away, while $u_{r}$ and $u_{\theta}$ oscillate around zero and around the laminar value, respectively. As the time advances further, the fluctuation and oscillation will eventually decay to zero. 


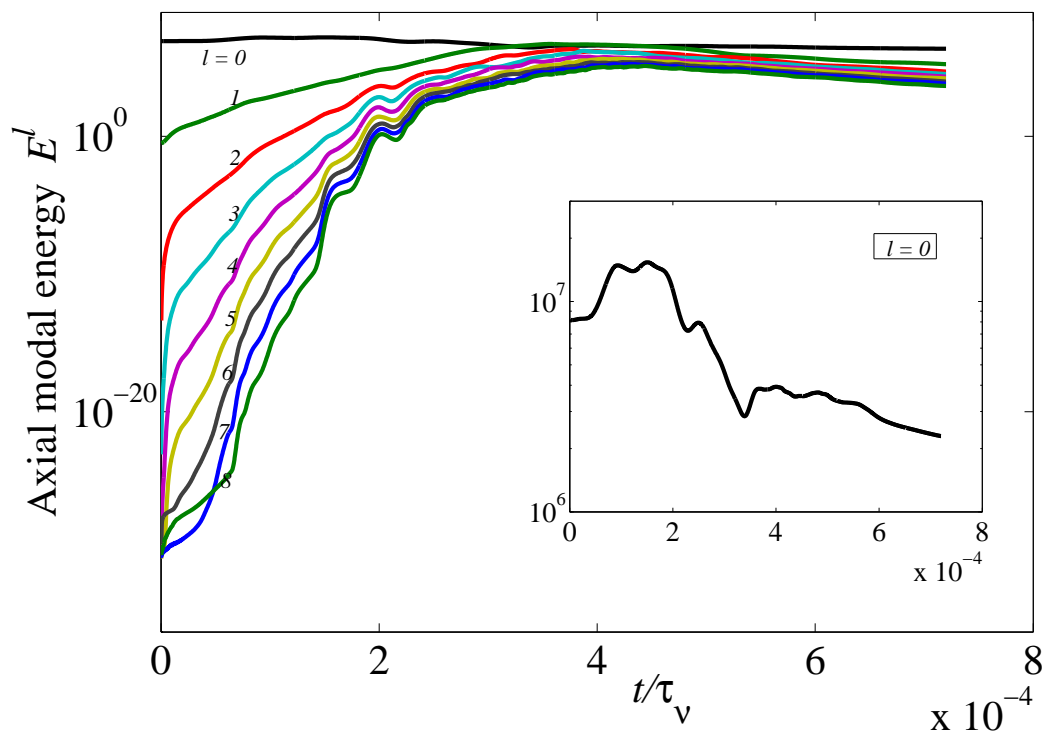

Figure 4.4: The temporal evolution of the axial modal energy $\left.E^{l}(t)\right|_{l=0} ^{8}$ at $R e_{i}=$ $1 \times 10^{5}$. The number on the line corresponds to the axial mode number. The inset shows the axial modal energy for $l=0$, the energy of the mean flow. The time is in the viscous unit $\tau_{\nu}=d^{2} / \nu$.
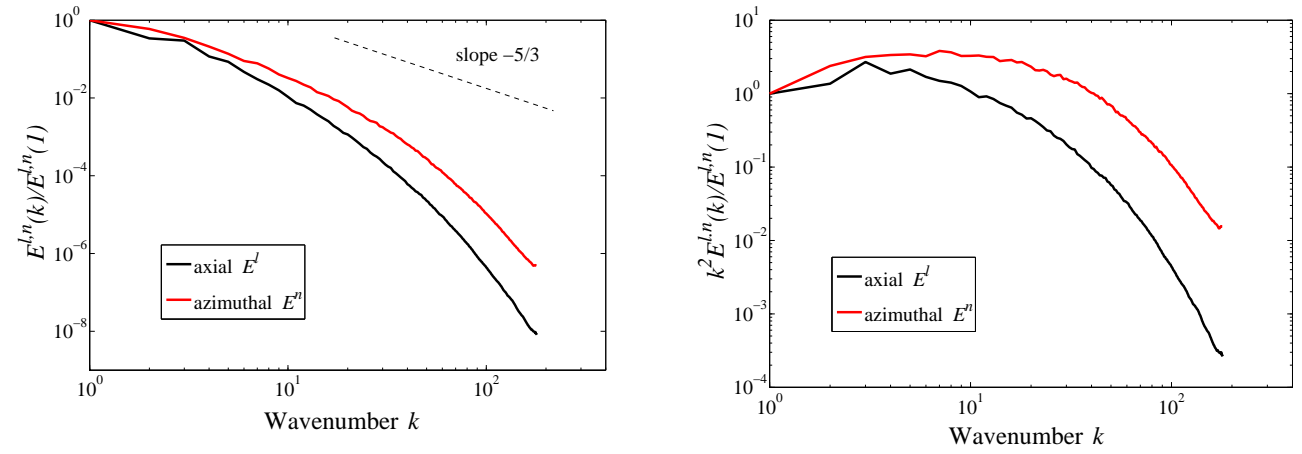

FiguRe 4.5: Axial and Azimuthal energy power $E^{l, n}(k)$ (left) and dissipation spectra $k^{2} E^{l, n}(k)$ (right), normalized by the energy of mode $1, E^{l, n}(1)$. The time is $t=$ $4 \times 10^{-4}$ and the Reynolds number is $R e_{i}=1 \times 10^{5}$. The guided line has a slope $-5 / 3$, the power cascade exponent for homogeneous isotropic turbulence.

The decay of turbulence can be more clearly seen in Fig. 4.7, the azimuthal and axial modal energy, $E^{n}(t)$ and $E^{l}(t)$, at $R e_{i}=1 \times 10^{5}$. The azimuthal and axial mode both varies from 0 to the highest 192 (half of the number of points) from top to bottom. Each mode is decaying about exponentially. Moreover, we visualize also the field of streamwise vorticity, $\omega_{\theta}=\partial_{z} u_{r}-\partial_{r} u_{z}$, at two different moments, as shown in Fig. 4.8 for $R e_{i}=1 \times 10^{4}$. The flow is rather turbulent at time $t_{1}$ while almost laminar at time $t_{2}$. Similar dynamical behaviors have been found at higher Re (Fig. 4.9). 
Chapter 4. Nonlinear transition to turbulence in quasi-Keplerian flow

\subsection{Discussion and summary}

By performing DNS simulations of axially periodic TCF in the quasi-Keplerian regime, decaying turbulence is found at shear Re up to $10^{5}$. Starting from the optimal perturbations (2D, axially independent) with 3D noise, the flow undergoes three stages before the relaminarization: $i$ ) linear transient growth; $i$ ) secondary instabilities due to nonlinear mechanisms; iii) turbulence decaying. The nonlinear secondary instabilities causes the linearly stable flow turbulent. However, turbulence does not sustain against the stabilizing (viscous or Coriolis) forces, oscillating down towards laminar motion. From the temporal evolution of axial or azimuthal modal energy, it is shown that turbulence decay at all Re starts at approximately the same viscous time, $\sim 4 \times 10^{-4} \tau_{\nu}$. This indicates that the viscous dissipation is the main mechanism for decay.

Decaying turbulence has been also found in recent studies from two other groups 111, 112]. Mónico et al. conducted also DNS simulations of TCF with periodic axial boundary conditions and found no sustained turbulence. A comparison to our simulations is drawn in Table 4.4. Although similar results have been obtained from both simulations, it is worth to mention that the resolutions per volume in their simulations are about 20 times smaller than ours at similar Re. However, as mentioned before, with such low resolutions our spectral code blows up, since the energy-dissipating scale is not well resolved. In their study, the second-order finite difference code may have high numerical viscosity, which helps dissipate the energy. In addition, their initial conditions are turbulent states taken from the Rayleigh-unstable regime (at stationary outer cylinder). A sudden rotation of the outer cylinder may cause very strong resistance of the flow, driving the intense initial turbulence even quicker relaminarization.

\begin{tabular}{ccc}
\hline \hline DNS & In this thesis & by Mónico et al. \\
\hline Highest $R e_{s}{ }^{1}$ & $1.01 \times 10^{5}$ & $9.45 \times 10^{4}$ \\
$\eta=r_{i} / r_{o}$ & 0.71 & 0.714 \\
Geometry $L_{r} \times L_{\theta} \times L_{z}$ & $1 \times 0.96 \times 0.5$ & $1 \times 2.614 \times 2.094$ \\
$\#$ points per unit length & $2048 \times 800 \times 1024$ & $800 \times 196 \times 490$ \\
\hline Numerical method & 2nd-order FD $(r)+$ & 2 nd-order FD \\
& Fourier spectral $(\theta, z)$ & $(r, \theta, z)$ \\
\hline Initial conditions & from optimal perturbations & turbulent states from \\
& + 3D noise & Rayleigh-unstable regime \\
\hline \hline
\end{tabular}

TABLE 4.4: A Comparison between our DNS simulations and the ones from Twente [11]

On the other hand, no persistent turbulence was observed at shear Re up to $2 \times 10^{6}$ in the new experiments of TCF conducted by Edlund and Ji [112]. With a radius ratio of $\eta \simeq 0.34$, the new experimental setup has axial end caps splitting into three

\footnotetext{
${ }^{1}$ The definition of $R e_{s}$ in this thesis is different from the one in [111] by a factor of $\frac{2}{1+\eta}$.

${ }^{2}$ The azimuthal length $L_{\theta}$ is based on the inner cylinder.
} 
parts: the middle part is flexible whereas the other two are attached with the inner and outer cylinder, respectively. By fine-tuning the rotation speed of the middle ring, the velocity profile of the laminar flow can match very well with the theoretical Couette profile. The laminar flow is then disturbed by injecting jets of fluid at the mid-plane. The decaying time scales of turbulence after stopping injection, however, are much shorter than what is observed in DNS simulations. A possible explanation is that in simulations the perturbations are imposed in the whole field whereas in experiments disturbances are always localized. The lack of strong spatial interactions in experiments may shorten the decay time, with a more stabilizing curvature effect $(\eta \simeq 0.34)$ also possibly contributing.

Albeit all these studies found no sustained hydrodynamic turbulence, the strong secondary instabilities observed in our simulations hints that subcritical hydrodynamic turbulence may still exist at higher Reynolds numbers. Besides, only the incompressible hydrodynamics of the flow is considered in these studies, which is very different from the astrophysical disks: i) The Reynolds number of astrophysical relevance is much higher; ii) Astrophysical flows are mostly compressible; iii) The physics of astrophysical disks is more complicated, including many other physical fields, such as self-gravity, magnetic field and temperature gradients [113]. Regarding the extremely high computing cost of DNS at higher Re, efforts in the near future may be better invested on the study of multi-physical processes, such as baroclinic instabilities originated from temperature gradients [114 116]. 
Chapter 4. Nonlinear transition to turbulence in quasi-Keplerian flow
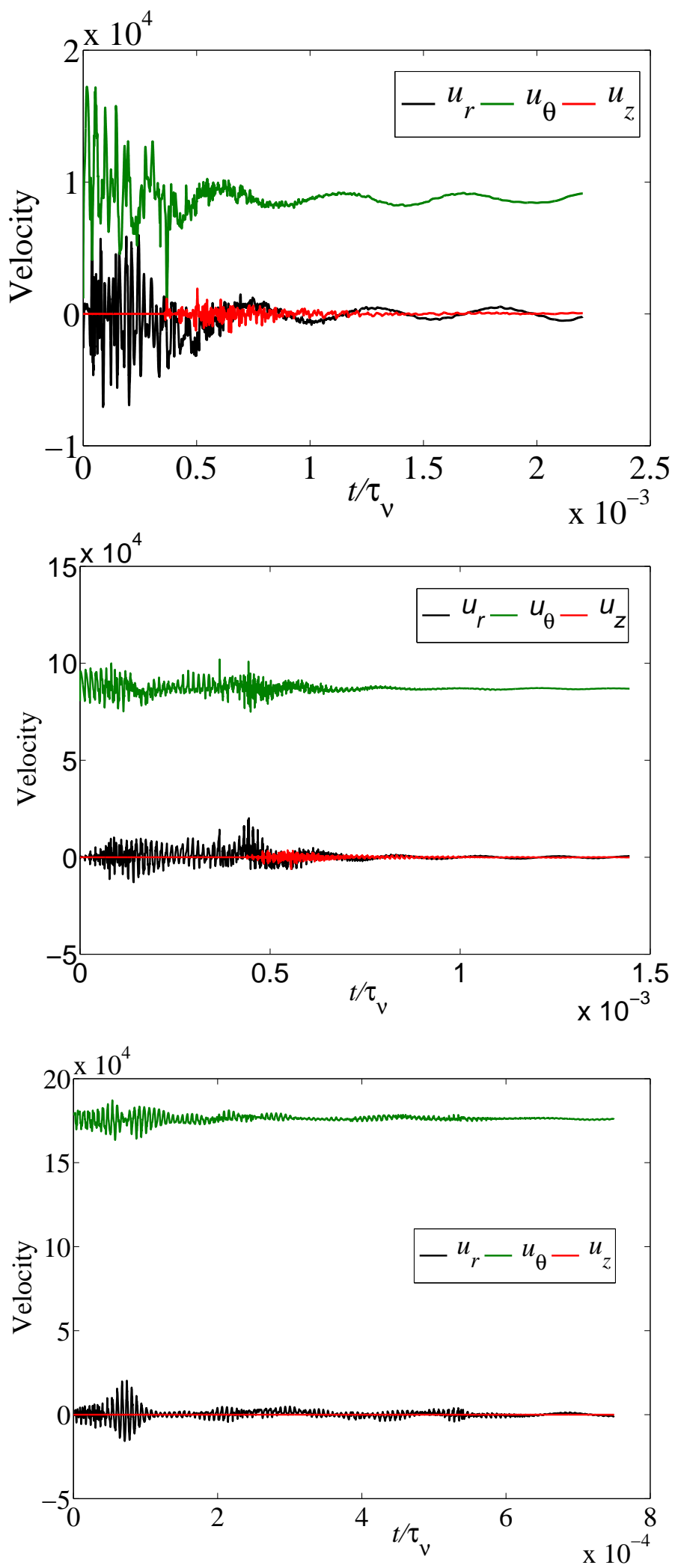

Figure 4.6: The temporal evolution of the velocity at a point at mid gap: $R e_{i}=$ $1 \times 10^{4}$ (top) $R e_{i}=1 \times 10^{5}$ (middle) and $R e_{i}=2 \times 10^{5}$ (bottom). The time is in viscous unit $\tau_{\nu}=d^{2} / \nu$. Note that the total time durations are different at different Re. 
Chapter 4. Nonlinear transition to turbulence in quasi-Keplerian flow
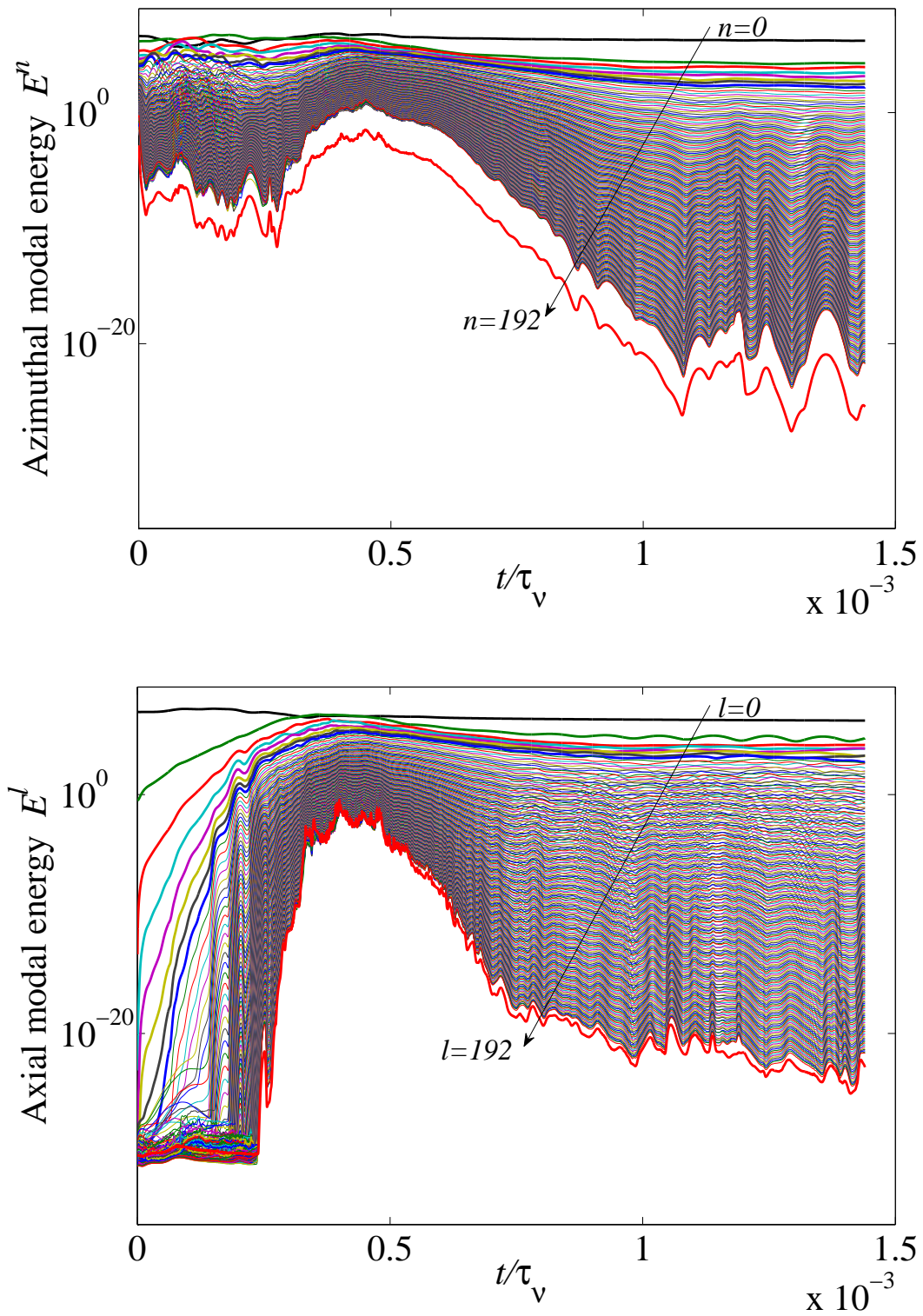

Figure 4.7: The temporal evolution of the azimuthal and axial modal energy, $E^{n}(t)$ (top) and $E^{l}(t)$ (bottom), at $R e_{i}=1 \times 10^{5}$. The bold lines are the first 8 modes and the last one. The time is in the viscous unit $\tau_{\nu}=d^{2} / \nu$. 

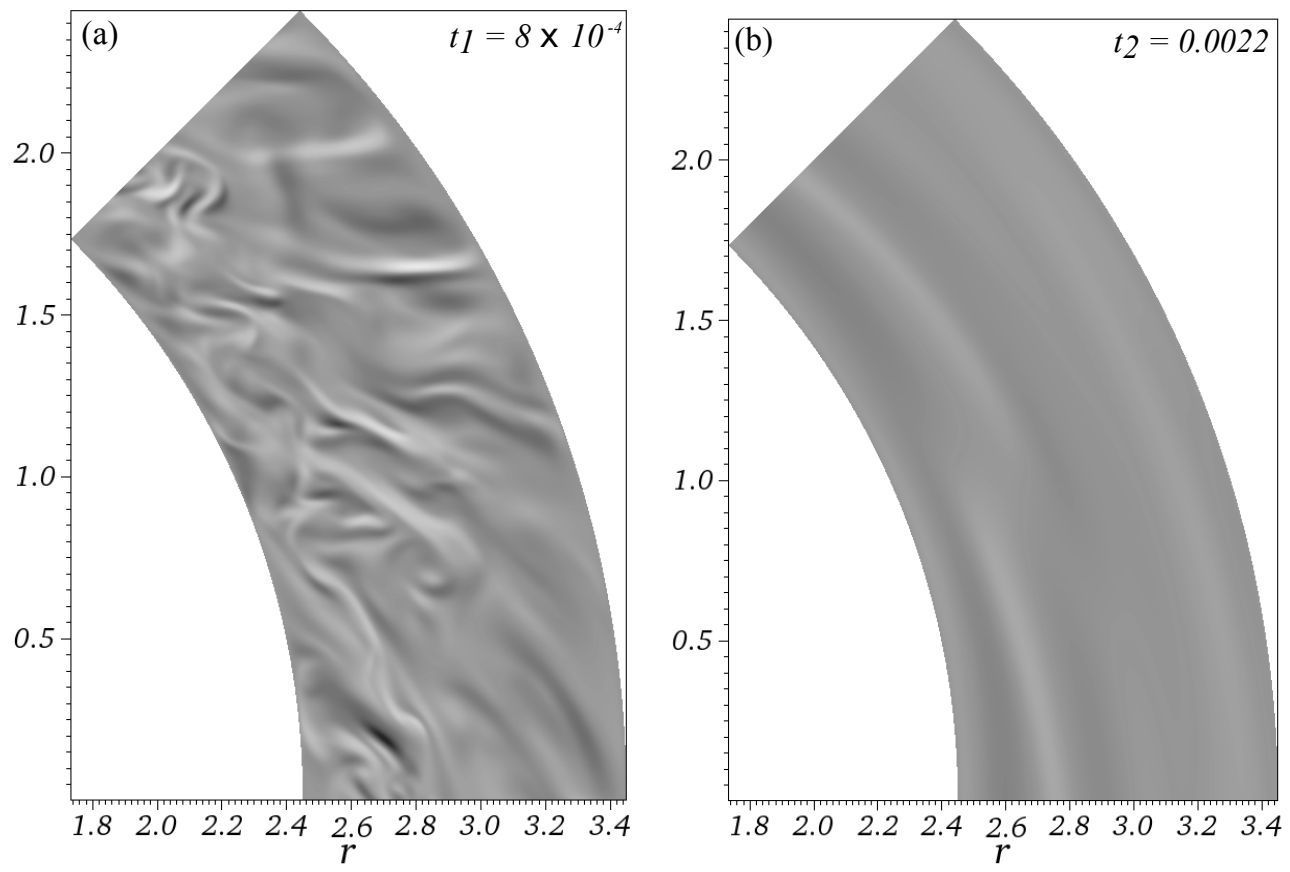

FiguRE 4.8: 2D contour plots of the streamwise vorticity $\omega_{\theta}$ at (a) $t_{1}=8 \times 10^{-4}$ and (b) $t_{2}=2.2 \times 10^{-3}$. The Reynolds number is $R e_{i}=1 \times 10^{4}$. The plot is in the $r-\theta$ plane for $z=d$.
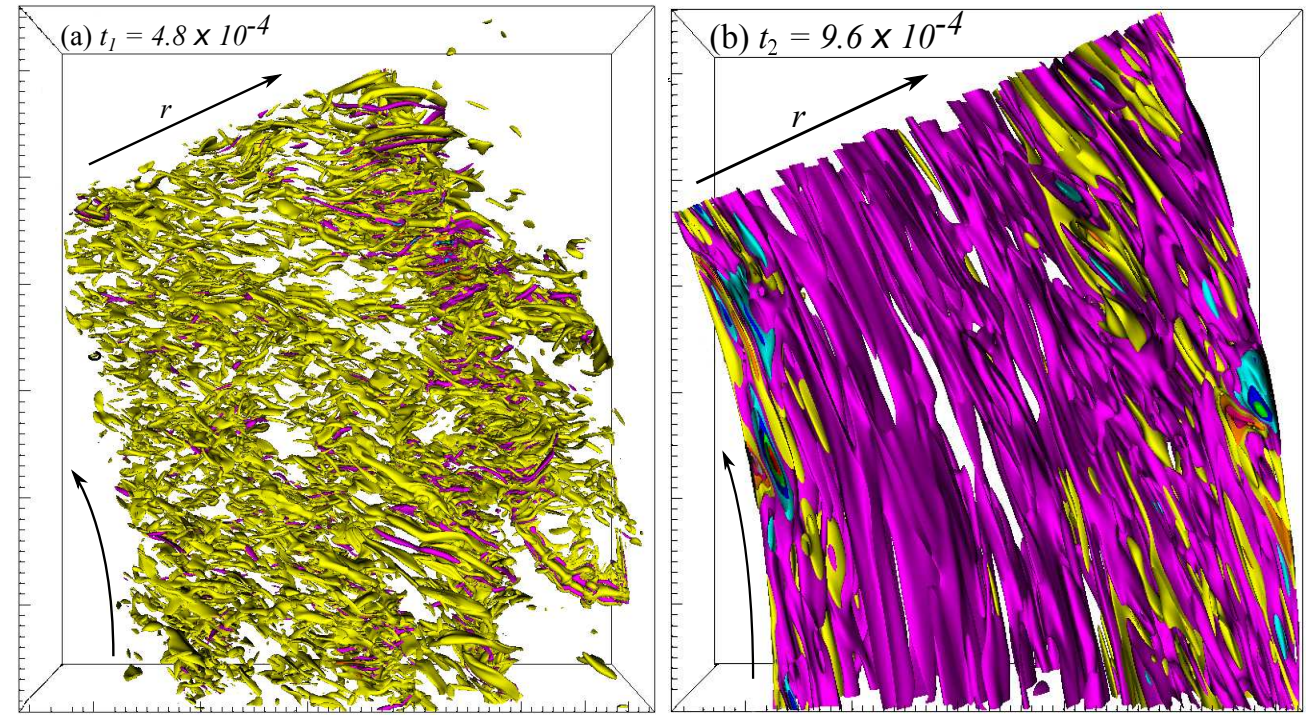

FiguRE 4.9: 3D contour plots of the streamwise vorticity $\omega_{\theta}$ at (a) $t_{1}=4.8 \times 10^{-4}$ and (b) $t_{2}=9.6 \times 10^{-4}$. The Reynolds number is $R e_{i}=1 \times 10^{5}$. The left-bottom arrow indicates the azimuthal direction. 
"Everything SHOUld Be MADE AS SIMPle AS POSSIBLE, BUT NOT SIMPLER."

Albert Einstein (1879 - 1955)

\section{Transient Growth of Couette FloW UNDER CORIOLIS FORCE 1}

In Taylor-Couette experiments, the axial end caps drive via shear the nearby fluids to rotate at the same speed as themselves. However the fluids far away from the end caps follow the ideal Couette profile, hence forming an axial gradient of azimuthal velocity and causing axial motions. This effect is known as Ekman pumping effect, mainly due to the presence of Coriolis forces. This is one source for the contradictory results mentioned in $\$ 1.4$ in TCF experiments. Another source that causes Ekman effects is the Earth's rotation, which brings another component of rotation perpendicular to the cylinder walls. The influence of this rotation is still unclear. In this Chapter, the simplest shear flow, PCF, with system wall-normal rotation is studied, including the linear stability analysis and transient energy growth.

\subsection{Introduction}

Ekman-Couette flow represents the flow between two sliding parallel walls, where the whole setup is subject to external rotation around the axis perpendicular to the walls. Fig. 5.1 shows schematically the geometry of the flow. In the extreme cases, the flow

\footnotetext{
${ }^{1}$ The content in this Chapter is mainly from the paper: L. Shi, B. Hof, A. Tilgner, Transient Growth of Ekman-Couette Flow, Phys. Rev. E, 89, 013001 (2014).
} 
becomes either plane Couette flow (PCF, if without rotation) or two well separated Ekman layers for large rotation rates. Because of the theoretical importance and the practical generality in planetary systems, these two canonical shear flows have both received enormous attention for the last decades and PCF under spanwise system rotation has also been widely studied [117, 118]. However, little work has been done to study the Ekman-Couette flow. Hoffmann et. al. [119] studied the secondary and tertiary flow states in Ekman-Couette flow while Ponty et. al. [120] investigated the onset of thermal convection between two shearing plates under the influence of external oblique rotation. Both studies focused mainly on the regime of moderate to large external rotation. The instability at small rotation has been yet payed little attention to. Since experimental flows on Earth are mostly subject to weak external rotation and the Earth's rotation has been reported to have measurable influences in many other flows [121 124], the influence of weak system rotation on the Couette flows will be here specially studied.

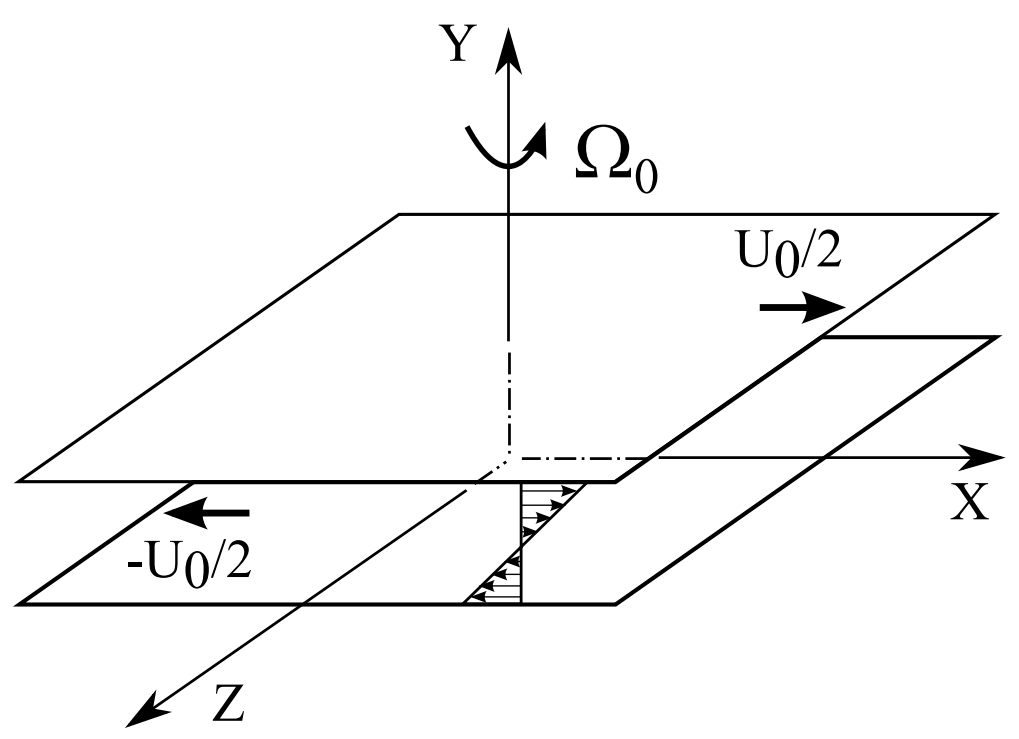

Figure 5.1: Schematic of Ekman-Couette flow. The top and bottom walls slide both with velocity $U_{0} / 2$ but in opposite directions along $\mathrm{X}$ axis. The whole setup rotates at a speed of $\Omega_{0}$ around the $\mathrm{Y}$ axis. The velocity profile corresponds to the base flow of plane Couette flow, $\left(U_{0} / 2 \cdot y, 0,0\right)$.

In this Chapter we present a study on the linear stability and the transient energy growth exploring a wide parameter space in Ekman-Couette flow. This work is interesting theoretically and is also motivated by recent conflicting results $[72,73,75,78,125]$ in astrophysical rotating flows, on whether turbulence in cold accretion disks can arise via hydrodynamic instabilities. The Ekman layers introduced by the top and bottom end walls in experimental Taylor-Couette setups influence remarkably the bulk flow and make the flow rather complicated. Besides, the Earth's rotation gives rise to another component of rotation, perpendicular to the rotation axis of the cylinders. At high Re, its effects may become non-negligible, except that the rotation axis of the 
cylinders aligns with the one of Earth's rotation. We here choose the simplest geometry to study the influence of the Ekman layer on the linearly stable flows. We find that in PCF an infinitesimal external rotation causes linear instabilities.

This Chapter is structured in the following way. The linearized Ekman-Couette problem is formulated mathematically in Section $\$ 5.2$, followed by the linear stability analysis in Section $₫ 5.3$. We finally study the transient energy growth in Section $4 \$ 5.4$.

\subsection{Problem formulation}

Considering that the fluid is incompressible, the governing equations of the fluid motion are the Navier-Stokes equations (here with external system rotation),

$$
\partial_{t} \mathbf{u}+\mathbf{u} \cdot \nabla \mathbf{u}+2 \Omega_{0} \mathbf{e}_{y} \times \mathbf{u}=-\frac{1}{\rho} \nabla p+\nu \Delta \mathbf{u}, \quad \nabla \cdot \mathbf{u}=0 .
$$

where $\mathbf{u}(\mathbf{x}, t)$ is the flow velocity field and $p(\mathbf{x}, t)$ is the pressure field. By taking the half gap distance between two plates $D / 2$ as the length unit and $D /\left(2 U_{0}\right)$ as the time unit,

$$
l=l^{\prime} \cdot D / 2, \quad t=t^{\prime} \cdot D /\left(2 U_{0}\right), \quad \mathbf{u}=\mathbf{u}^{\prime} \cdot U_{0}, \quad p=p^{\prime} \cdot \rho U_{0}^{2},
$$

we obtain the non-dimensional form of Eq. 5.1,

$$
\partial_{t} \mathbf{u}+\mathbf{u} \cdot \nabla \mathbf{u}+\frac{1}{\mathrm{Ro}} \cdot \mathbf{e}_{y} \times \mathbf{u}=-\nabla p+\frac{1}{\mathrm{Re}} \cdot \Delta \mathbf{u}, \quad \nabla \cdot \mathbf{u}=0 .
$$

with the Reynolds number and the Rossby number

$$
\operatorname{Re}=\frac{U_{0} D}{2 \nu}, \quad \operatorname{Ro}=\frac{U_{0}}{\Omega_{0} D}
$$

Note that the non-dimensional symbols in Eq. 5.2 are omitted. We define another nondimensional parameter, the rotation number $\Omega=\frac{\Omega_{0} D^{2}}{\nu}$. Here, Ro $=\frac{2 \mathrm{Re}}{\Omega}$. Considering the boundary conditions and the symmetry property about the plane $y=0$, the base velocity profile has the form of $[U(y), 0, W(y)]$. We introduce the complex function $Z(y)=U(y)+i W(y)$ and yield

$$
Z(y)=\frac{1}{2} \frac{e^{i \gamma y}-e^{-i \gamma y}}{e^{i \gamma}-e^{-i \gamma}}
$$

with $\gamma=\sqrt{\frac{\mathrm{Re}}{\mathrm{Ro}}} \frac{1+i}{\sqrt{2}}$ (see Appendix $\mathrm{C}$ for details). Fig. 5.2 displays the base velocity profile at $\mathrm{Re}=1000$, with and without rotation, respectively. At $\Omega=50$, the external rotation distorts qualitatively the base flow such that the inflection points appear in the profiles. 


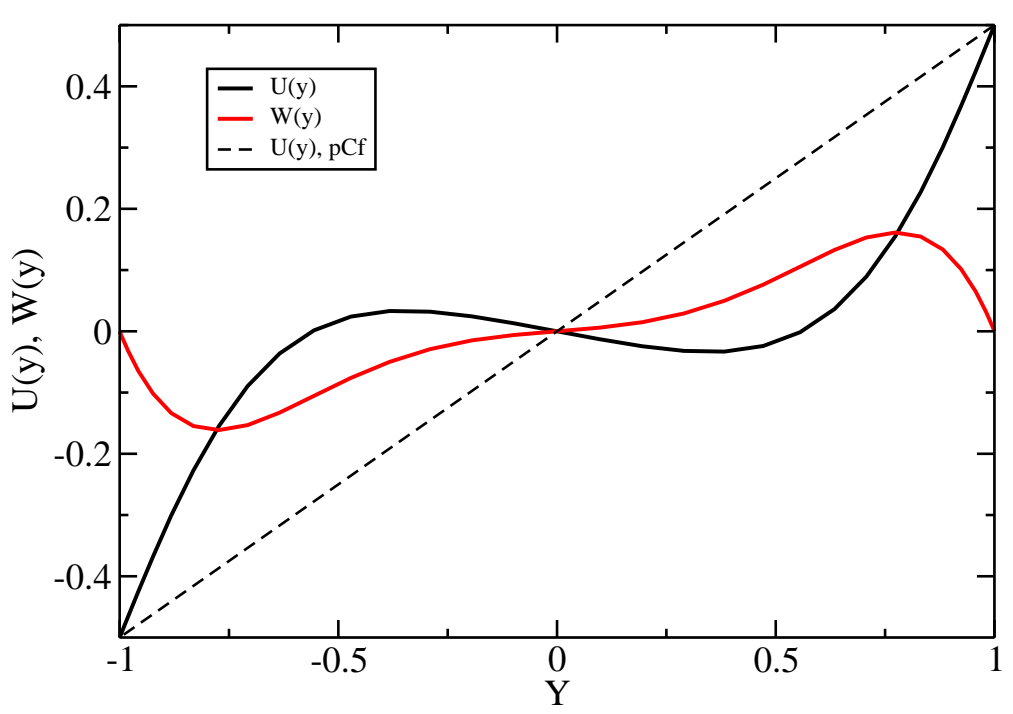

Figure 5.2: Base velocity profiles at $\operatorname{Re}=1000, \Omega=50$ (solid and dash-dotted lines) and at $\operatorname{Re}=1000, \Omega=0$ (dashed line).

To study the linear stability and transient dynamics of the base flow, we decompose the velocity field as $\mathbf{u}=\mathbf{u}_{\text {pert }}+\mathbf{U}_{\text {base }}$, where $\mathbf{U}_{\text {base }}=[U(y), 0, W(y)]$. Let $v$ and $\eta$ denote the perturbation of the wall-normal velocity and vorticity. By taking the curl once and twice, respectively, of Eq. 5.2 and then projecting into the Y direction, we obtain the linearized equations for the perturbation variables $(v, \eta)$,

$$
\begin{array}{r}
\partial_{t} \nabla^{2} v+\left(U \partial_{x}+W \partial_{z}\right) \nabla^{2} v-W^{\prime \prime} \partial_{z} v-U^{\prime \prime} \partial_{x} v= \\
\frac{1}{\operatorname{Re}} \nabla^{4} v-\frac{1}{\mathrm{Ro}} \partial_{y} \eta \\
\partial_{t} \eta+\left(U \partial_{x}+W \partial_{z}\right) \eta+U^{\prime} \partial_{z} v-W^{\prime} \partial_{x} v= \\
\frac{1}{\operatorname{Re}} \nabla^{2} \eta+\frac{1}{\mathrm{Ro}} \partial_{y} v
\end{array}
$$

In this paper we focus on the following modal perturbation,

$$
v=\hat{v}(y, t) e^{i(\alpha x+\beta z)}, \quad \eta=\hat{\eta}(y, t) e^{i(\alpha x+\beta z)},
$$


where $\alpha$ and $\beta$ are the wavenumbers in $X$ - and $Z$ - direction, respectively. By inserting into Eq. 5.4, we have the modal equations,

$$
\begin{aligned}
\partial_{t} \hat{\nabla}^{2} \hat{v}= & -i(U \alpha+W \beta) \hat{\nabla}^{2} \hat{v}+i\left(U^{\prime \prime} \alpha+W^{\prime \prime} \beta\right) \hat{v} \\
& +\frac{1}{\operatorname{Re}} \hat{\nabla}^{4} \hat{v}-\frac{1}{\mathrm{Ro}} \partial_{y} \hat{\eta}, \\
\partial_{t} \hat{\eta}= & -i(U \alpha+W \beta) \hat{\eta}-i\left(U^{\prime} \beta-W^{\prime} \alpha\right) \hat{v} \\
& +\frac{1}{\operatorname{Re}} \hat{\nabla}^{2} \hat{\eta}+\frac{1}{\mathrm{Ro}} \partial_{y} \hat{v},
\end{aligned}
$$

with $\hat{\nabla}^{2}=\partial_{y}^{2}-\left(\alpha^{2}+\beta^{2}\right)$.

Through the Chebyshev spectral discretization in the spatial direction [126], the above partial differential equations is transformed into a linear system $\partial_{t} \hat{\mathbf{v}}=-i L \hat{\mathbf{v}}$, where $\hat{\mathbf{v}}=$ $[\hat{v}, \hat{\eta}]$. The linear stability and transient growth is then calculated by the eigenvalues and eigenvectors of the linear operator $L$, which is computed in this paper by the subroutines in the LAPACK library. The accuracy and convergence of the method have been verified against the results in [126].

\subsection{Linear instability}

The inflection points in the base profile hint that the Ekman-Couette flow may be linearly unstable. Thus we first investigate the linear instability of the flow. The range of parameters under study is $\operatorname{Re} \in[100,300000]$ and $\Omega \in[0,100]$. A bisection method is employed to find the critical curve $\operatorname{Re}^{c}(\Omega)$, separating the linearly stable and unstable regions. The results are shown in Fig. 5.3. At small $\Omega(\Omega<5)$, the linear instability is here referred to as type " 0 " and the critical Reynolds number $\mathrm{Re}^{c}$ is found to scale with $\Omega$ as $\operatorname{Re}^{c}(\Omega) \simeq 1800 \cdot \Omega^{-1}$. Therefore, as $\Omega \rightarrow 0, \operatorname{Re}^{c} \rightarrow \infty$, which is consistent with the linear stability of plane Couette flow $(\Omega=0)$ at any Re [127, 128]. As $\Omega$ is increased, we recover the type I and type II instabilities previously found in Ekman layer flow [129]. The corresponding wavenumbers are shown in Fig. 5.4. Here the wavenumber $k=\sqrt{\alpha^{2}+\beta^{2}}$ and the angle $\theta=-\arctan (\alpha / \beta)$, where $\theta$ is the angle between the wavevector $\mathbf{k}$ and the $Z$ axis. The negative sign indicates anti-clockwise direction. As shown in the Fig. 5.4, type I instability is characterized by a large wavenumber and a negative angle while type II has a smaller wavenumber and a positive angle. The results agree very well with the ones previously reported in [119, 120]. 


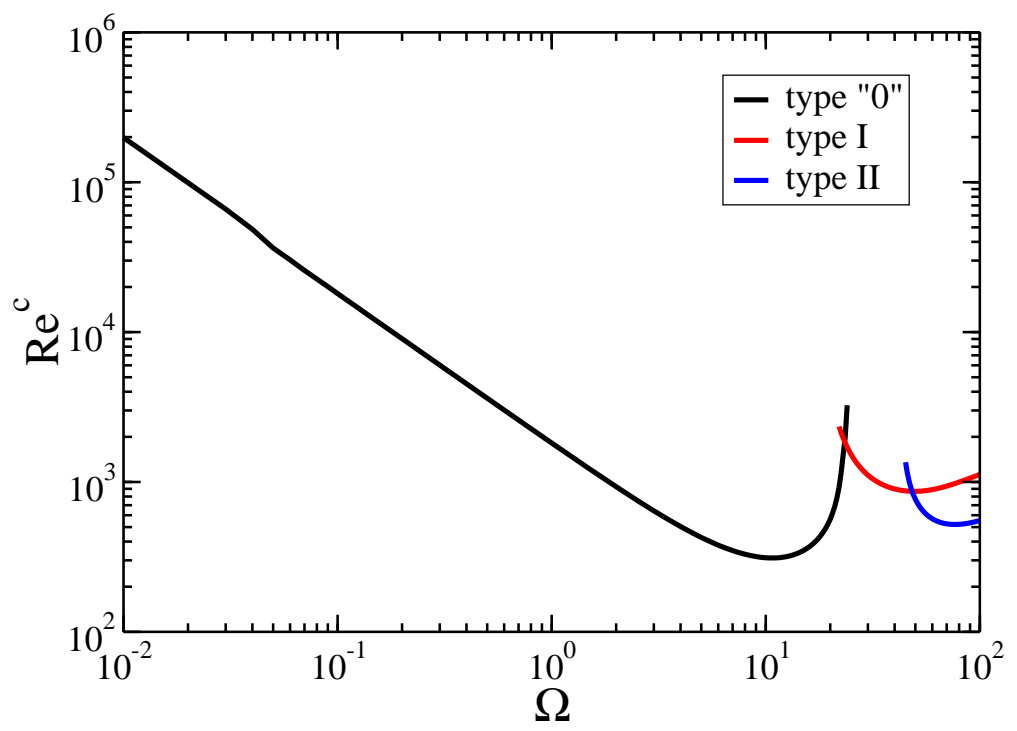

Figure 5.3: Critical Reynolds number $\operatorname{Re}^{c}$ as a function of $\Omega$. For $\Omega<5$, the critical Re scales inversely with the system rotation, $\operatorname{Re}^{c} \simeq 1800 \cdot \Omega^{-1}$.
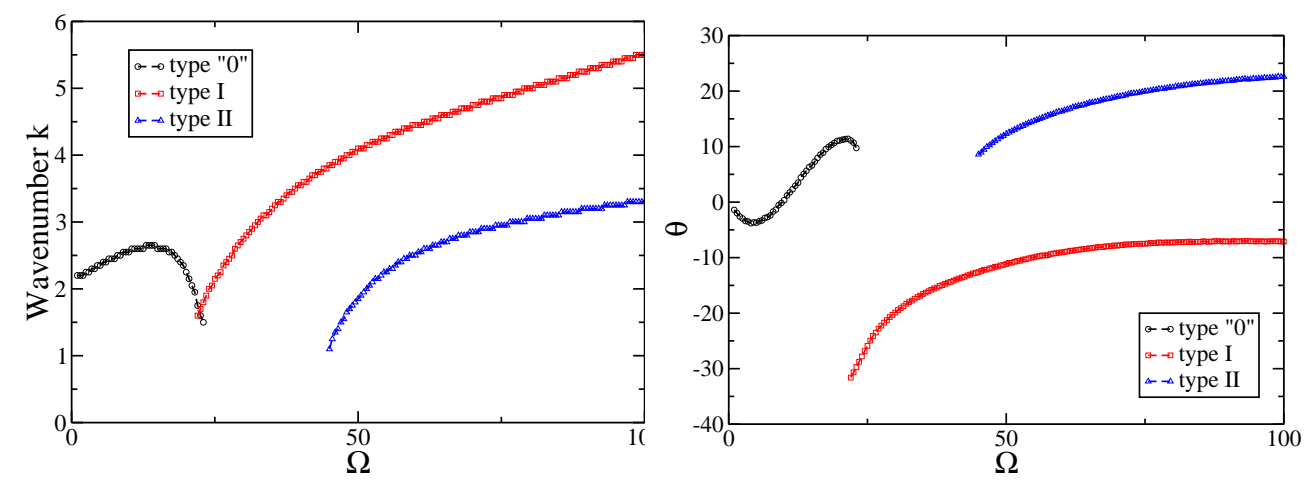

Figure 5.4: The variation with $\Omega$ of the wavenumber $k=\sqrt{\alpha^{2}+\beta^{2}}$ (top) and the angle $\theta$ (bottom).

\subsection{Transient growth}

Below the neutral stability curve $\operatorname{Re}^{c}(\Omega)$, the flow is linearly stable and the transient growth of initial perturbations may play an important role in the nonlinear transition to turbulence. Due to the non-normality of the governing linear operator L, PCF undergoes substantial transient growth before nonlinear interaction sets in, [126, 130]. However, the influence of the external system rotation on the transient behavior is still unknown. Here we employ the method presented in [126] to compute the optimal transient growth and the optimal perturbations. Let us first define the physical quantities 

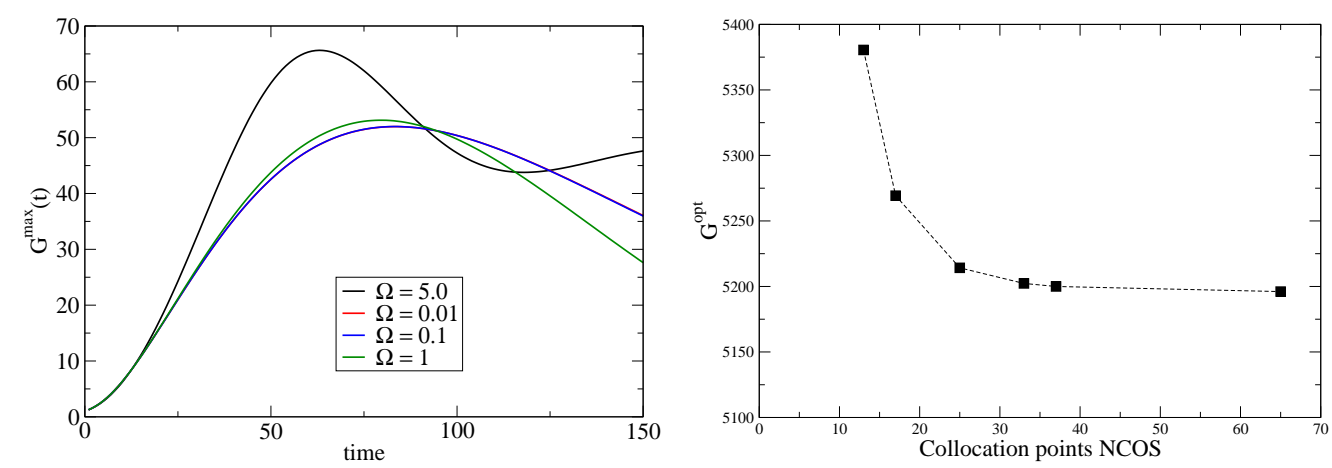

Figure 5.5: (left) The temporal evolution of the maximum transient growth rate $G^{m}(t)$ at $R e=500$ and at different rotation rates $\Omega$. At $\Omega=5.0$, the flow is already linearly unstable. (right) Convergence of the global optimal growth rate $G^{\text {opt }}$ at $R e=5000$ and $\Omega=0.05$. The collocation points $N C O S=33$ is chosen for the following computations.

of interest, the spectral energy

$$
\hat{E}(\alpha, \beta, \operatorname{Re}, \Omega ; t)=\|\hat{\mathbf{v}}\|^{2}=\int_{-1}^{1}\left(\left|\partial_{y} \hat{v}\right|^{2}+k^{2}|\hat{v}|^{2}+|\hat{\eta}|^{2}\right) d y
$$

and the optimal growth function

$$
G(\alpha, \beta, \operatorname{Re}, \Omega ; t)=\sup _{\hat{\mathbf{v}}(\cdot ; 0) \neq 0} \frac{\hat{E}(\cdot ; t)}{\hat{E}(\cdot ; 0)}=\sup _{\hat{\mathbf{v}}(\cdot ; 0) \neq 0} \frac{\|\hat{\mathbf{v}}(\cdot ; t)\|}{\|\hat{\mathbf{v}}(\cdot ; 0)\|}
$$

The spectral energy measures the kinetic energy contained in the mode $(\alpha, \beta)$, while the optimal growth function is the maximal energy growth achievable among all possible initial perturbations within time $t$. As presented in [126], the growth function $G(\cdot ; t)$ can be directly computed by the eigenvalues and eigenvectors of the linear operator $L$. For simplicity of notation, we here introduce two additional functions: the maximal growth in time as $G^{m}(\alpha, \beta, \operatorname{Re}, \Omega)=\max _{t} G(\alpha, \beta, \operatorname{Re}, \Omega ; t)$ and the global optimal growth in the $(\alpha, \beta)$ plane as $G^{\text {opt }}(\operatorname{Re}, \Omega)=\max _{\alpha, \beta} G^{m}(\alpha, \beta, \operatorname{Re}, \Omega)$. Examples of the time evolution of $G^{m}$ is shown in Fig. 5.5 (left), while the right figure shows the convergence of $G^{o p t}$ at $R e=5000$ and $\Omega=0.05$. One property of $G^{m}$ is the symmetry under the transformation $(\alpha \rightarrow-\alpha, \beta \rightarrow-\beta)$. The maximal growth $G^{m}$ in the $\alpha-\beta$ plane at various Re and $\Omega$ is shown in Fig. 5.6 , evidencing the symmetry with respect to the point $(0,0)$. The Reynolds number and the rotation number are fixed in each case. The range in the $\alpha-\beta$ plane is $[-10,10] \times[-10,10]$. At small $\Omega$ (Fig. $5.6 \mathrm{a})$, the contour plot of $G^{m}$ is similar to that in plane Couette flow [126], where the maximum is located very close to the $\beta$ axis. As $\Omega$ increases, the effect of the external rotation becomes non negligible and the maximum moves away from the $\beta$ axis. Moreover, increasing Re from 500 (Fig. 5.6a) to 1500 (Fig. 5.6b) results in a substantial increase of $G^{m}$ while increasing $\Omega$ from 0.05 (Fig. [5.6 a) to 50 (Fig. [5.6 d) leads to a sharp decrease in $G^{m}$. It is worthwhile to note that the modes that achieve the maximal transient growth are 
not the least unstable modes computed from the linear stability analysis.
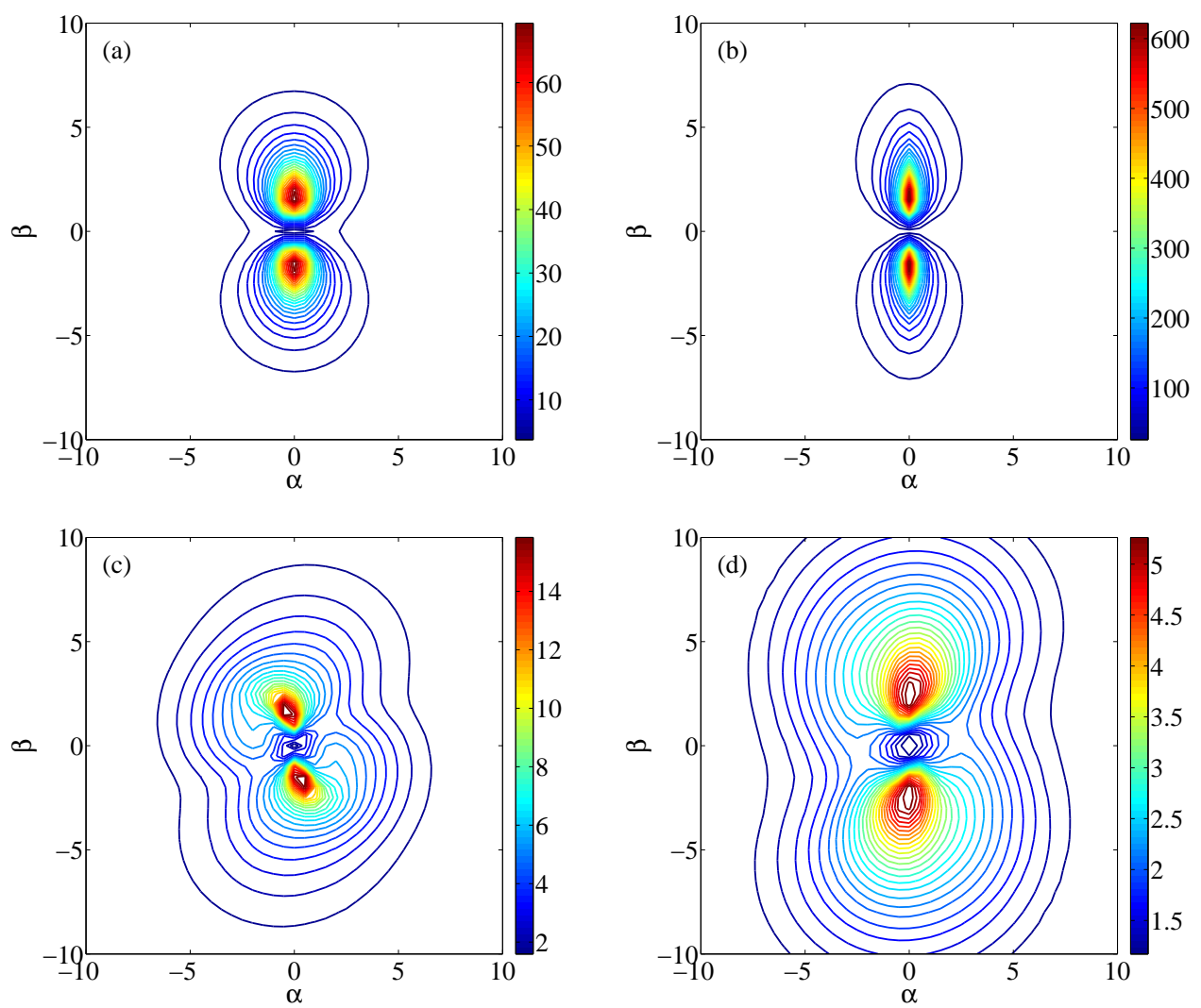

Figure 5.6: Contour plot in $(\alpha, \beta)$ space of the global maximum growth rate $G^{m}$ : (a) $\operatorname{Re}=500, \Omega=0.05$; (b) $\operatorname{Re}=1500, \Omega=0.050$; (c) $\operatorname{Re}=500, \Omega=20$; (d) $\operatorname{Re}=500, \Omega=50$. The size of $G^{m}$ is indicated by the color value.

We further compute the global optimal growth function $G^{\text {opt }}(\operatorname{Re}, \Omega)$ in the linearly stable region in $\operatorname{Re} \in[0,100]$ and $\Omega \in[0,1500]$. The search of the global maximum in the $\alpha-\beta$ plane is done by the downhill simplex method [131]. Fig. [5.7 shows the contour plot of $G^{\text {opt }}(\operatorname{Re}, \Omega)$. The highest growth is located in the left top region with low $\Omega$ and high Re, while the lowest growth is in the right bottom region with high $\Omega$ and low Re. Nevertheless, the middle bumps in the contour plot shows that the growth variation is not monotonic, whereas the non-smooth, irregular patches are due to the lack of sufficiently high resolution. Quantitatively, the scaling of $G^{\text {opt }}$ with Re and $\Omega$ is shown in Fig. 5.8, Fig. 5.8 displays the variation of $G^{o p t}(\operatorname{Re}, \Omega)$ with Re at fixed $\Omega$. The optimal growth scales at small $\Omega$ slightly faster than a power law with Re, $G^{\text {opt }} \sim \mathrm{Re}^{2}$, whereas the power-law scaling disappears at large $\Omega$ and the transient growth becomes much smaller than the one at small $\Omega$. Fig. $5.8 \mathrm{~b}$ plots the growth $G^{o p t}$ as a function of $\Omega$ when Re is fixed. It can be seen that the transient growth is enhanced with weak external rotation while it is dramatically suppressed as $\Omega>25$.

The transient growth in rotating Couette flow is finally compared to the case without external rotation. Here in the rotating case we choose $\Omega=0.05$. For a plane Couette 


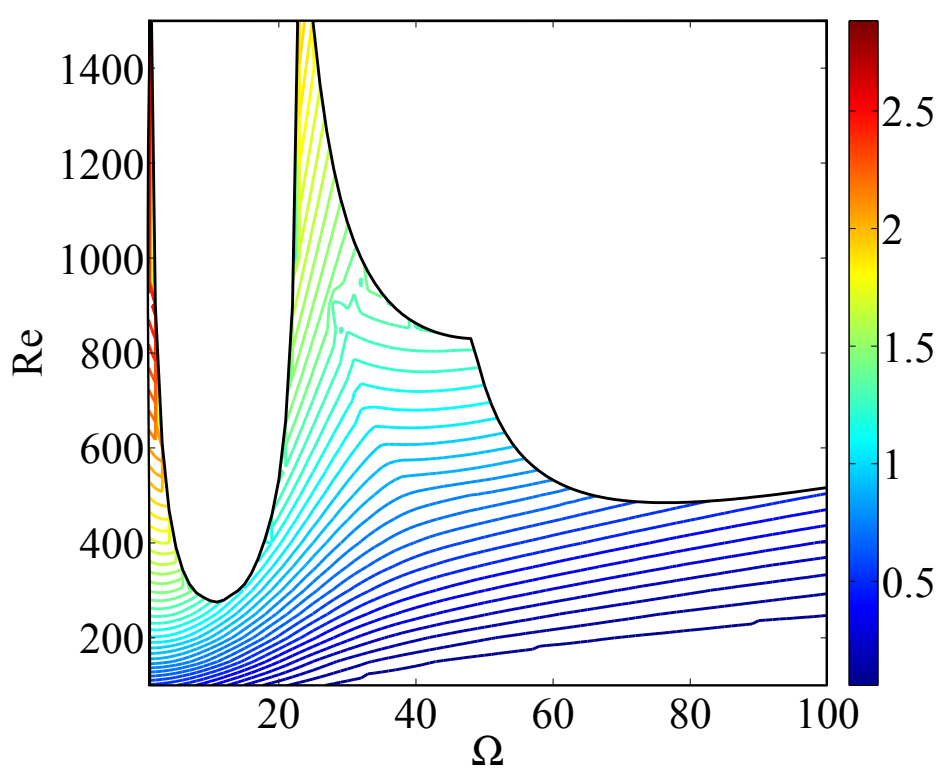

Figure 5.7: Contour plot of the global optimal growth $G^{o p t}$ in the $\Omega$-Re plane. The boundary (the black line) is the neutral curve from the linear stability analysis. The color value is on a logarithmic scale, e. g., the value "2" denotes $G^{\text {opt }}=10^{2}$.

setup in Göttingen (Germany) with a gap distance $D \simeq 0.03 m$, the rotation number induced by the Earth's rotation $\Omega_{0} \simeq 7.3 \cdot 10^{-5} \cdot \sin (51.32 \cdot \pi / 180) \simeq 5.7 \cdot 10^{-5}$ is $\Omega=\frac{\Omega_{0} D^{2}}{\nu_{H_{2} O} \mathrm{O}} \simeq 0.0513$, where the water viscosity is $\nu_{H_{2} \mathrm{O}} \simeq 10^{-6} \mathrm{~m}^{2} / \mathrm{s}$ at $T=20^{\circ}$. The Reynolds number under investigation is in the range $\operatorname{Re} \in[1500,35000]$. The results are ploted in Fig. 5.9, In PCF, we have the optimal transient growth $G^{o p t} \simeq 1.18 \times 10^{-3} \mathrm{Re}^{2}$ (Fig. 5.9 a) achieved at time $t^{\text {opt }} \simeq 0.117$ Re (Fig. 5.9 b), which agrees perfectly with the results in 130]. The corresponding wavenumber $\alpha^{\text {opt }}$, as shown in Fig. [5.9k, scales as $\alpha^{\text {opt }} \sim \operatorname{Re}^{-1}$ and $\beta^{\text {opt }}$ (Fig. $5.9 \mathrm{~d}$ ) stays constant, $\beta^{\text {opt }} \simeq 1.60$. For the case with external rotation $\Omega=0.05$, the transient growth is slightly increased, with a power exponent little greater than 2.0 and it is obtained at an earlier moment (see Fig. 5.9b). The wavenumber $\alpha$ is basically the same as the case without rotation, while the wavenumber $\beta$ decreases linearly with Re and has a different slope at different $\Omega$. Furthermore, as shown in Fig. 5.10, the optimal perturbations are both in the form of inclined roll structures. However, the elongated rolls in the case of $\Omega=0.05$ are slightly twisted.

\subsection{Discussion and summary}

We presented in this Chapter a study of the linear stability and transient energy growth in rotating plane Couette flows, where the rotation axis is perpendicular to the planes. Such a rotating framework is of interest to geophysical and astrophysical flows. For example plane Couette and Taylor Couette experiments that are often used to study 
Chapter 5. Transient growth of Couette flow under Coriolis force
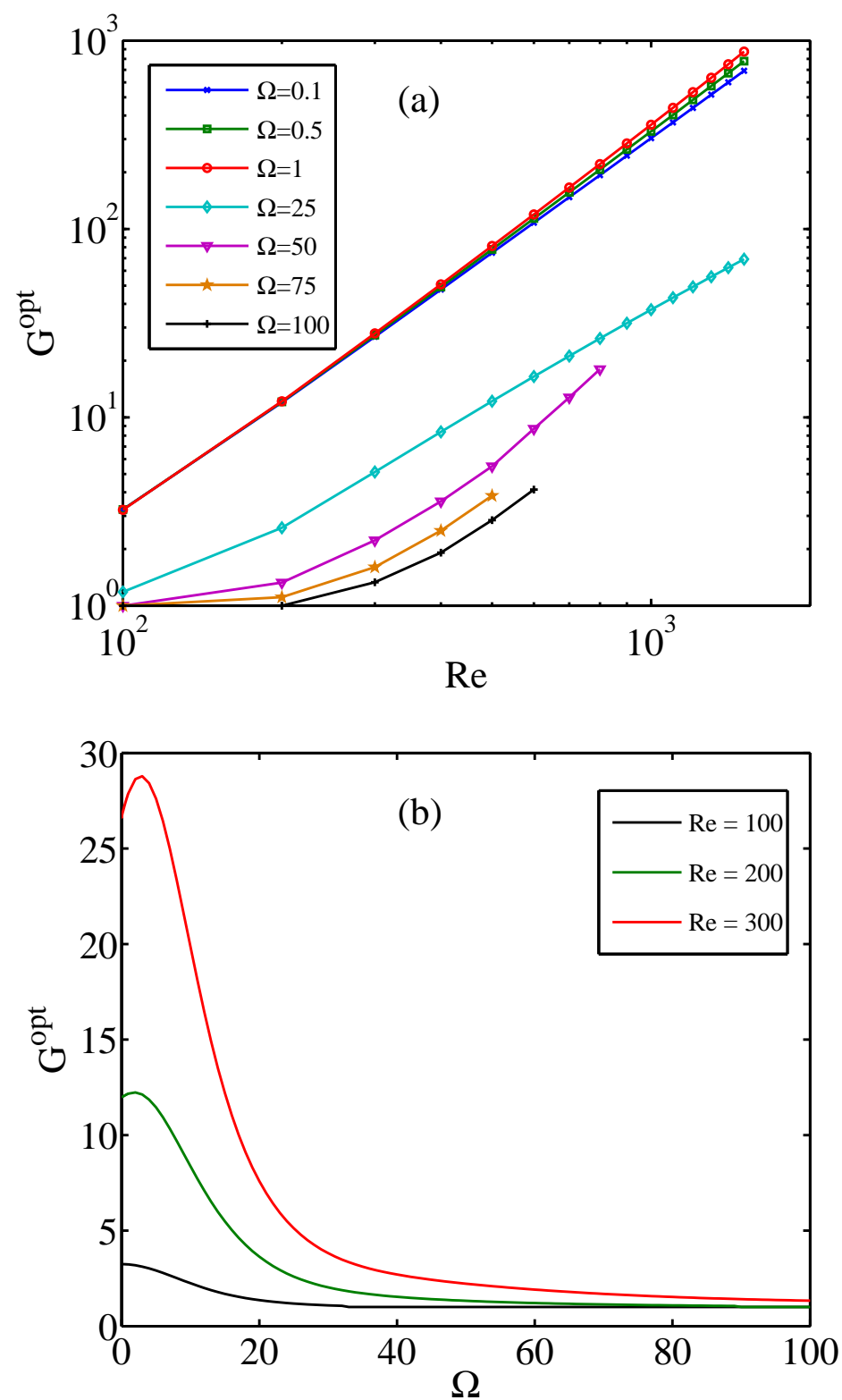

Figure 5.8: Scaling of $G^{\text {opt }}$ (a) with Re at different $\Omega$ and (b) with $\Omega$ at different Re.

the stability of geophysical and astrophysical flows [72] are all exposed to the Earth's rotation. By linearizing the Navier-Stokes equations, we firstly computed the neutral stability curve dividing the linearly stable and unstable region in the Re- $\Omega$ parameter space. Three different type of instabilities are found: for $\Omega>20$, type I and type II instabilities which have been already known from the Ekman boundary layer flow and, for $\Omega<20$, type " 0 " instabilities. The results are consistent with the previous one reported in [119, 120]. Moreover, we found that the critical Re for $\Omega<5$ scales as a power law with $\Omega, \operatorname{Re}^{c}(\Omega) \simeq 1800 \cdot \Omega^{-1}$, which agrees with the fact that the PCF $(\Omega=0)$ is linearly stable for all Re. 

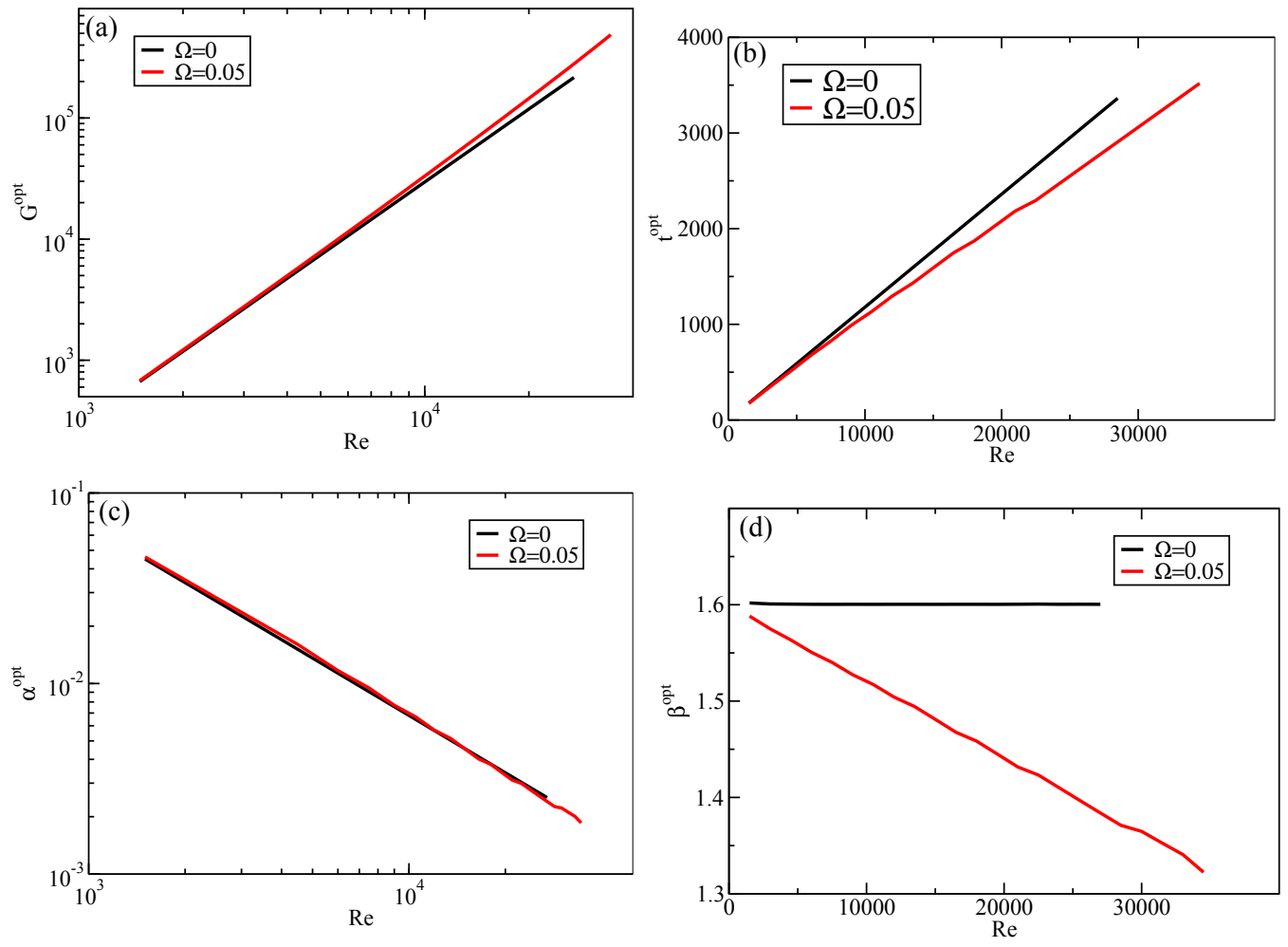

Figure 5.9: Scaling with Reynolds number at $\Omega=0$ and $\Omega=0.05$. (a) The global mamixum $G^{o p t} \sim \mathrm{Re}^{-2}$; (b) The corresponding time where the global maximum is attained, $t^{\text {opt }}=a+b \cdot \operatorname{Re}$; (c) Wavenumber $\alpha^{\text {opt }}(\operatorname{Re}) \sim \operatorname{Re}^{-1}$, which is almost the same at different $\Omega$; (d) Wavenumber $\beta^{\text {opt }}(\mathrm{Re})=c+d \cdot \operatorname{Re}$, with different slopes at different $\Omega$.
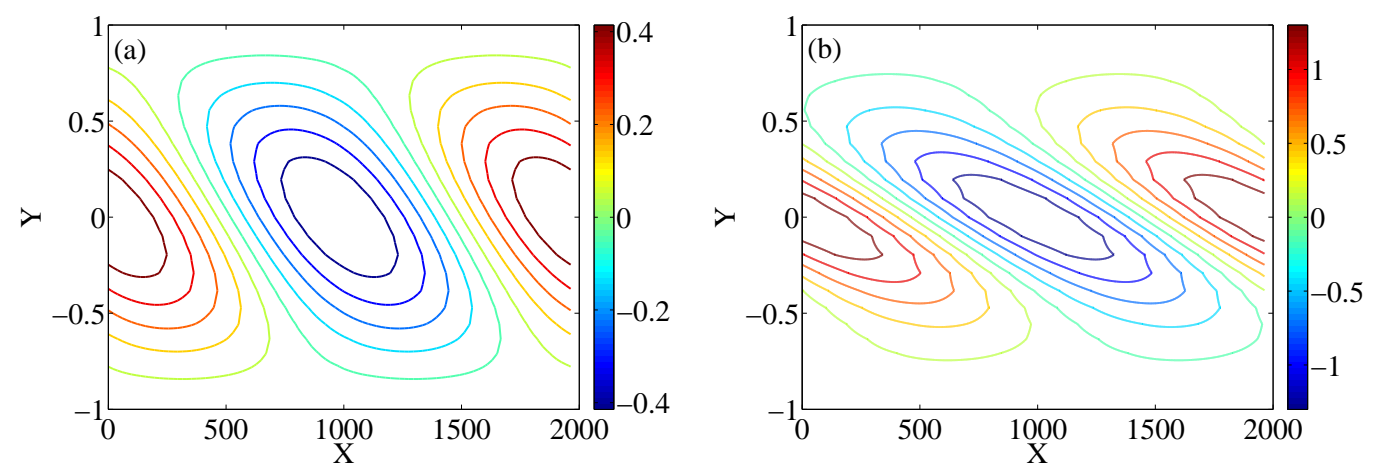

Figure 5.10: Contour plot of the optimal perturbation $v^{\text {opt }}(x, y, z=0)$ for (a) $\Omega=0$ and (b) $\Omega=0.05$. The Reynolds number is $\operatorname{Re}=20000$. The wavenumbers are the ones giving the optimal transient growth.

Through computation of the eigenvalues and eigenfunctions of the governing linear operator $L$, we obtained the global optimal transient growth in the $\alpha-\beta$ plane amongst all possible initial perturbations. Our results show that the external rotation can have both enhancing and suppressing effects on the optimal transient growth. For weak rotation, it increases the transient growth while strong rotation inhibits significantly the transient growth. At the rotation numbers relevant for geophysical applications, for 
example the atmospheric boundary layer, the transient growth is so small that linear stability analysis appears to be the appropriate tool to determine the stability limits of Ekman layers in the geophysical context. At small rotation the optimal growth scales slightly faster than the power law $\Omega^{2}$ as is found in plane Couette flow. Furthermore, the wavenumbers where the optimal transient growth is obtained is also different from the non-rotating case. The optimal wavenumber $\alpha$ stays the same, scaling as a power law $\alpha \sim \operatorname{Re}^{-1}$, whereas the optimal wavenumber $\beta$ is shifted linearly with Re.

\begin{tabular}{lcccr}
\hline \hline place & gap $d / \mathrm{mm}$ & $\Omega$ & $\mathrm{Re}^{c \text {,linear }}$ & $d^{\star} / \mathrm{mm}$ \\
\hline Toronto [132] & 58 & 0.169 & 10651 & 234.8 \\
Stockholm [23] & 10 & 0.006 & $3 \times 10^{5}$ & 214.8 \\
Paris [39] & 7 & 0.003 & $6 \times 10^{5}$ & 212.7 \\
Zürich [133] & 31.2 & 0.052 & 34615 & 227.7 \\
\hline \hline
\end{tabular}

TABLE 5.1: Existing experimental setups of plane Couette flows and their onset of linear instability under Earth's rotation. The value $\operatorname{Re}^{c}$ is computed according to $\operatorname{Re}^{c} \simeq 1800 \cdot \Omega^{-1}$, while $d^{\star}$ corresponds to the gap distance beyond which the linear instability sets in before the nonlinear transition to turbulence in PCF.

Mostly, the rotation of the Earth has been intuitively considered to be too weak to influence the experiments qualitatively. However, our results tell us that in the case of PCF the Earth's rotation does change radically the flow stability, from linearly stable to linearly unstable. This instability may be attributed to the inflection points in the base velocity profile introduced by the external rotation. Table 5.1 lists the existing experimental PCF setups and their approximate critical Reynolds number for the linear instability under Earth's rotation. The value $d^{\star}$ indicates a reference gap distance where $\operatorname{Re}^{c, \text { linear }}=\operatorname{Re}^{c, \text { nonlinear }}, i$. e., the critical Reynolds number from the linear instability equals the one computed from nonlinear mechanism in PCF ( 650 based on the gap distance, see [39, 43, 103]). Although the linear $\operatorname{Re}^{c}$ are far beyond the onset of turbulence via nonlinear mechanism, the results provide important theoretical guidance for the design of future PCF setups. It may also be relevant to recent TaylorCouette studies at Re of order $\mathcal{O}\left(10^{6}\right)[72,73,125]$, in that at large Re the additional component of rotation induced by the Earth's rotation may also cause inflection points in the base velocity profile. Further studies on the underlying physical mechanisms will contribute to the understanding of shear flows in rotating frameworks. 
"When I MEeT GOD, I AM GOING TO ASK HIM TWO QUESTIONS: Why RELATIVITY? AND WHy TURBULENCE? I REALLY BELIEVE HE WILL HAVE AN ANSWER FOR THE FIRST."

Werner K. Heisenberg (1901-1976)

\section{6 \\ Conclusions}

Subcritical transition to turbulence arises in many circumstances (e.g. in oil pipes and other wall-bounded flows) and is a very interesting and active research topic for fluid dynamicists. Subcritical turbulence is characterized by strong spatial inhomogeneity and rich dynamical complexity, which goes beyond the framework of classical bifurcation and temporal chaos theory. In this thesis we developed a highly efficient DNS code for simulating Taylor-Couette flow, which is parallelized by a hybrid MPIOpenMP strategy and has an excellent scalability up to $3 \times 10^{4}$ cores. With this code we firstly studied statistically the subcritical transition to turbulence in a slender domain in plane Couette flow. The results provide strong evidence that subcritical transition to turbulence is a continuous phase transition and falls into the DP universality class, which brings important insight to the understanding of the nature of turbulence. We then performed DNS simulations in the quasi-Keplerian regime in TC flow in order to probe the existence of subcritical turbulence. At shear Re up to $1 \times 10^{5}$, turbulent motion of fluids is observed due to the secondary instabilities and nonlinear mechanisms. However, turbulence does not sustain and eventually decays. Finally, the influence of external system rotation (such as Earth's rotation) on linearly stable PCF was studied. It was found that external system rotation causes linear instability and strongly inhibits the transient growth at large rotation rates. 


\subsection{Results and discussion}

The first part of the thesis focus on the onset of sustained turbulence in PCF. The main results are summarized as follows:

- Decay and splitting of localized turbulence are stochastic and memoryless dynamical processes, strongly depending on the initial conditions.

- The probability distributions of lifetime and splitting time of localized turbulence are exponential at each Re of this study.

- The mean lifetime and splitting time scale super-exponentially with Re.

- A lower bound for the critical $\operatorname{Re}, R e_{c}^{L B} \simeq 325$, is given by balancing the mean time scales of decay and splitting events.

- The width of turbulent stripes falls on the same exponential distribution at all Re of this study. The characteristic width is about $41 h$.

- At criticality, the flow displays scale invariance: the laminar spacing obeys a power-law scaling, with an exponent $\sim-1.75$.

- The mean turbulent fraction is well approximated as $\bar{F}_{t} \sim\left(R e-R e^{c}\right)^{\beta}$, with $\beta \simeq 0.276$.

- The critical Reynolds number for sustained turbulence is $R e^{c}=328.5 \pm 0.5$.

These results are new and robust for the long slender and tilted PCF system (see Fig. (3.2), and are a breakthrough in understanding the nature of transition to turbulence and have significant theoretical meaning for the statistical physics. The dynamics in such a system is quasi-one dimensional: the turbulent stripe can either decay or spread only along the $Z$ axis. This system has much affinity with pipe flow in all aspects. In pipe flow, the turbulent puffs of about fixed length have the same memoryless dynamics. The probability distributions and scalings of lifetime and splitting time are qualitatively comparable. Although the large mean time scales of the events in pipe flow makes a quantitative study at criticality beyond current reach, the model of [134] predicted that the transition in pipe flow also belongs to the 1D-DP universality class. Beyond the similarity one should also bear in mind the differences: 1) Unlike in pipe flow where the new puff originates from the front edge of the old one and develops in the downstream, the stripe in Couette flow splits as a whole and can appear in both sides of the old stripe; 2) At high Re, a puff progressively grows in length and develops into a slug, whereas in Couette flow the stripes have more or less fixed width and disappear in the turbulent environment as Re is increased above certain value. 
One may argue that this system does not capture the full spectrum of rich dynamics in an infinite PCF system. However, we believe that the results presented above are still valid in extended $\mathrm{PCF}$ systems, except that the transition will belong to the 2DDP universality class instead of 1D. First, our slender titled box involves the essential coherent structures (streamwise vortices and streaks) and dynamical events (decay and spreading) which are necessary for turbulence to sustain. Second, the long slender box takes into account of the large spatial correlation length at criticality and hence avoids the finite-size effect. Third, our obtained critical Reynolds is consistent with the value previously reported in experiments [39] and simulations [43]; Finally, the mean turbulent fraction in counter-rotating TCF where the system is characterized by the spiral turbulent stripes is reported to have the same scaling as in the 2D-DP universality class [13].

In the second part of the thesis we probed subcritical hydrodynamic turbulence in quasi-Keplerian flows by performing highly resolved DNS simulations of TCF. Due to limited available computing time, we have done simulations only at three points in parameter space, with the highest shear Reynolds number up to $10^{5}$. In all our simulations, the flow is disturbed initially by the optimal linear perturbations, undergoes a secondary instability due to nonlinear mechanisms and becomes turbulent. However, turbulence decays and would relaminarise. The results may be also valid in the whole quasi-Keplerian regime in TCF. Our main conclusion is that no sustained hydrodynamic turbulence exists in quasi-Keplerian regime at Re up to $10^{5}$ and that other physical processes may be required to explain the origin of turbulence in astrophysical accretion disks.

The above simulations have been performed in the Garching Supercomputer Center (Rechnungszentrum Garching, RZG), with a high-performance DNS code . This code is designed to simulate TCF, solving the NS equations in cylindrical coordinates. For the spatial discretization, the spectral Fourier-Galerkin method is employed in the azimuthal and axial directions, whereas finite differences are used in the radial direction. The nonlinear terms are treated by the pseudospectral technique. A second-order, semi-implicit projection scheme is adopted for the time integration. The code is validated in many flow regimes: laminar flow, time-dependent wavy vortex flows, transitional and turbulent flows. To achieve higher Re, the code is parallelized with a hybrid MPI-OpenMP strategy. This strategy is simple to implement, reduces inter-node communications and is more efficient compared to a flat-MPI parallelization. The code demonstrates very good scalability up to $3 \times 10^{4}$ cores and hence enables to simulate TCF at Re relevant to experiments.

In the last part of the thesis, we studied the influence of external system rotation on linearly stable shear flow. For simplicity we investigated the linear stability and the transient growth behaviour of the PCF under external rotation perpendicular to 
the parallel wall, which is often called Ekman-Couette flow. We summarize the main results as follows:

- External rotation causes linear instability in PCF.

- At small external rotation rate, the critical Reynolds number for linear instability varies inversely with the rotation rate, $R e^{c, l i n e a r} \simeq 1800 \Omega^{-1}$.

- The maximum transient growth rates are strongly inhibited at large external rotation.

Note that we have wall-normal system rotation instead of spanwise system rotation. Since all experiments on Earth are subject to the Earth rotation, our results show that the PCF setups on Earth are all linearly unstable. Although the critical Reynolds numbers for linear instability under earth's rotation are mostly far beyond the one from the nonlinear mechanism $R e^{c, \text { nonlinear }}$ ( see Table 5.1), the linear instability itself is interesting theoretically and may arise in other shear flows experiments on earth.

\subsection{Future work}

One interesting and difficult direction is to understand the physical mechanism of the localized turbulent stripes in PCF or TCF, including the growth process from localized turbulent spots to stripes, the splitting process of turbulent stripes, the fixed width $(\sim 20 d)$ of the stripes and the obliqueness of the laminar-turbulent interfaces and the selection of the inclined angle. Moreover, two turbulent stripes do not merge. All these behaviors of localized stripes have been repeatedly observed in either experiments or numerical simulations. There are some works in this direction [107, 135, 136]. However the physical mechanisms remain largely unknown. The understanding of the underlying physical mechanisms will help better interpret the results presented in this thesis.

Concerning the origin of turbulence in accretion disks, pure hydrodynamical nonlinear mechanisms are shown to hardly generate sustained turbulence at Re of order $\mathcal{O}\left(10^{5}\right)$. Since astrophysical accretion disks are complicated, with the presence of many physical fields such as gravity, thermal and magnetic fields, one may add these fields to the experiments or simulations. Baroclinic instability is an example due to the presence of thermal gradients, in the radial and axial (vertical) direction.

Another direction is the study of warped accretion disks, where the disks are distorted geometrically. The distortion of geometry of the disk introduces inflection points in the base velocity profile and hence may cause linear instabilities. Linear instabilities in warped accretion disks have already been reported in two recent papers [137, 138]. Nonlinear 3D simulations will bring valuable insights to the problem. 
"We prove What We Want to Prove, AND the REAL DIFFICULTY IS TO KNOW WHAT WE WANT TO PROVE."

Émile Chartier (1868 - 1951)

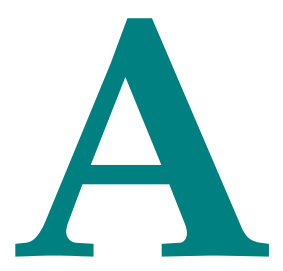

\section{COMPUTing THE AZIMUTHAL WAVE SPEED OF WAVY VORTICES IN TCF}

In Taylor-Couette flow, wavy vortices are time-dependent (periodic or quasi-periodic) flow states bifurcated from stationary Taylor vortices as the shear flow rate increases. The contour plot in Fig. 2.5 illustrates the wavy vortices flow state obtained at $R e_{i}=$ 458.1, $R e_{o}=0$ and $\eta=0.868$. Starting from the laminar Couette profile with small perturbation, the flow experiences firstly the Taylor vortices and after short-time adjustment it stabilizes at the stage of wavy vortices, which is demonstrated by the velocity evolution at one point in the flow as shown in Fig. A.1. From this figure we can roughly estimate the periodicity $T \simeq 0.045 \cdot d^{2} / \nu$. Since the azimuthal wavenumber $k_{\theta}$ of these wavy vortices is 6 , then the non-dimensional rotation speed is $\omega=\frac{2 \pi}{k_{\theta} T \Omega_{i}} \simeq \frac{2 \pi}{k_{\theta} T(1-\eta) R e_{i} / \eta} \simeq 0.334$. In this appendix, we present a more rigorous method to precisely evaluate the rotation speed of the waves.

This method consists in minimizing the difference between two velocity fields at two different time. Let $\mathbf{u}(\mathbf{r}, t)$ and $\mathbf{u}^{\prime}(\mathbf{r}, t+\Delta t)$ denotes the velocity fields at time $t$ and $t+\Delta t$. Due to the periodicity of the wavy vortices, these two velocity fields a priori collapse each other if we apply a backward phase shift $\Delta \theta$ in the azimuthal direction on $\mathbf{u}^{\prime}$. Mathematically, we need to find an appropriate $\Delta \theta_{0}$ such that the 2-norm of the velocity difference $\mathcal{E}(\Delta \theta)=\left\|\mathbf{u}(r, \theta, z, t)-\mathbf{u}^{\prime}(r, \theta-\Delta \theta, t+\Delta t)\right\|$ is minimal. In this way, the rotation speed $\omega$ equals $\left|\Delta \theta_{0} / \Delta t\right|$. Theoretically $\mathcal{E}(\Delta \theta)$ vanishes at $\Delta \theta=\Delta \theta_{0}$. 


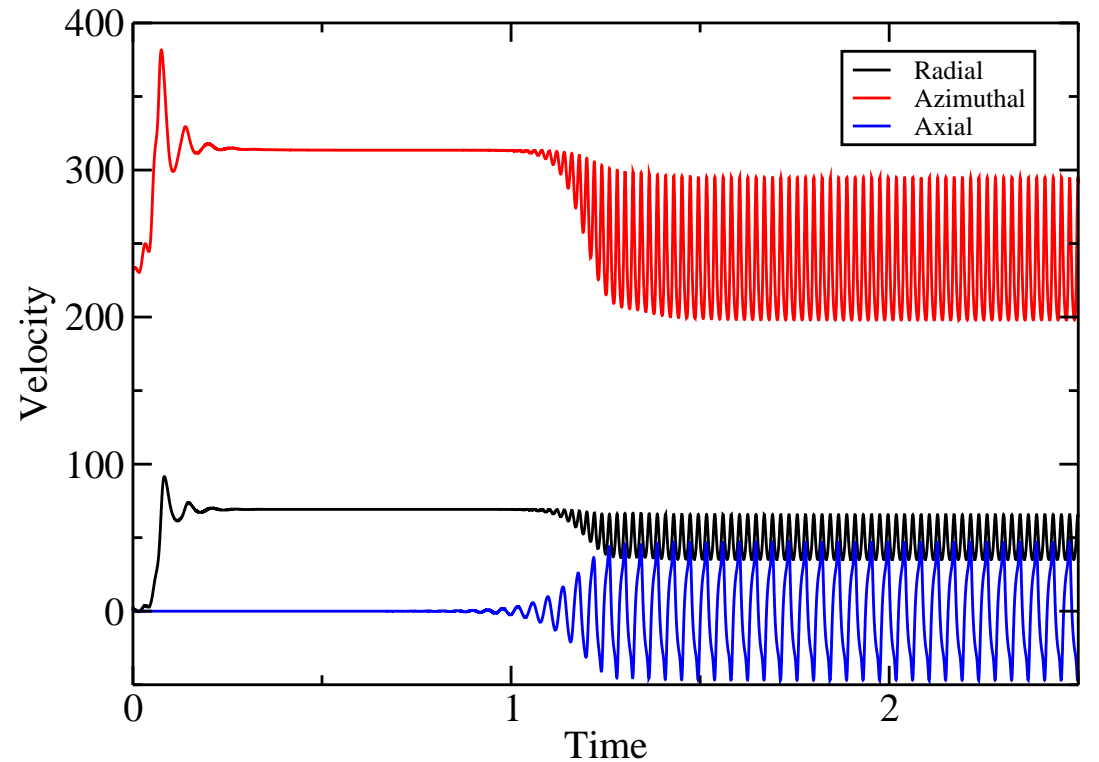

Figure A.1: Temporal evolution of the velocity at a middle point in the flow $\left(R e_{i}=\right.$ $458.1, R e_{o}=0$ and $\left.\eta=0.868\right)$. The parallel horizontal lines indicate the stage of stationary Taylor vortices while the final wavy curves signify the wavy vortices state. Time is in viscous time unit $d^{2} / \nu$.

By inserting equation (2.7) into the expression of $\mathcal{E}(\Delta \theta)$, we have

$$
\begin{aligned}
\mathcal{E}(\Delta \theta) & =\left\|\sum_{l=-L}^{L} \sum_{n=-N}^{N}\left(\hat{\mathbf{u}}_{l n}(r, t)-\hat{\mathbf{u}}_{l n}^{\prime}(r, t+\Delta t) e^{-i n k_{\theta} \Delta \theta}\right) e^{i\left(l k_{z} z+n k_{\theta} \theta\right)}\right\| \\
& =\sum_{l=-L}^{L} \sum_{n=-N}^{N}\left\|\left(\hat{\mathbf{u}}_{l n}(r, t)-\hat{\mathbf{u}}_{l n}^{\prime}(r, t+\Delta t) e^{-i n k_{\theta} \Delta \theta}\right) e^{i\left(l k_{z} z+n k_{\theta} \theta\right)}\right\| \\
& =\sum_{l=-L}^{L} \sum_{n=-N}^{N}\|\underbrace{\hat{\mathbf{u}}_{l n}(r, t)-\hat{\mathbf{u}}_{l n}^{\prime}(r, t+\Delta t) e^{-i n k_{\theta} \Delta \theta}}_{\equiv \Delta \hat{\mathbf{u}}_{l n}(r, \Delta \theta)}\| \\
& =\sum_{l=-L}^{L} \sum_{n=-N}^{N} \int_{r_{i}}^{r_{o}}\left\|\Delta \hat{\mathbf{u}}_{l n}(r, \Delta \theta)\right\| r d r
\end{aligned}
$$

where $\Delta \hat{\mathbf{u}}_{l n}(r, \Delta \theta) \equiv \hat{\mathbf{u}}_{l n}(r, t)-\hat{\mathbf{u}}_{l n}^{\prime}(r, t+\Delta t) e^{-i n k_{\theta} \Delta \theta}$ and can be evaluated easily in Fourier space, given that $\Delta \theta, \hat{\mathbf{u}}(r, t)$ and $\hat{\mathbf{u}}^{\prime}(r, t+\Delta t)$ are known. Hence the function $\mathcal{E}(\Delta \theta)$ can also be conveniently computed. We further employ a revised version of the Brent's local minimization algorithm [139] to find the minimal value of $\mathcal{E}(\Delta \theta)$. The used method combines the golden section search and the successive quadratic interpolation, which attains a super-linear convergence.

This method was applied to compute the wave speed in the above-mentioned case. We chose two velocity fields apart by a time interval $\Delta t=5 \times 10^{-3} d^{2} / \nu$. Fig. A.2 shows 


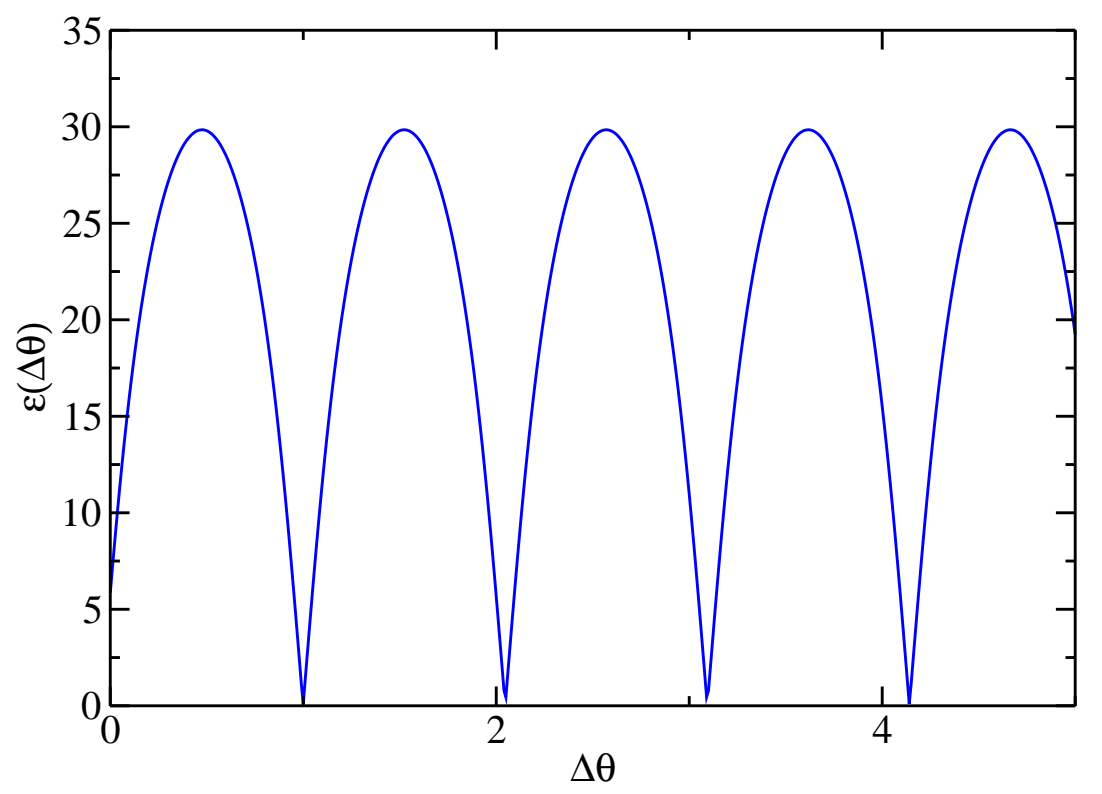

Figure A.2: The 2-norm of the velocity difference $\mathcal{E}$ as a function of the backward phase shift $\Delta \theta$.

the periodic variation of $\mathcal{E}(\Delta \theta)$ with $\Delta \theta$. The periodicity $\lambda$ of $\mathcal{E}(\Delta \theta)$ corresponds to the wavelength of the wavy vortices. Hence the wavenumber $k_{\theta}=2 \pi / \lambda \simeq 6$, which is consistent with the observation. The minimization routine also returned the first minima $\Delta \theta_{\text {min }}=0.9992$ and the value of $\mathcal{E}\left(\Delta \theta_{\text {min }}\right) \simeq 1.76 \cdot 10^{-4}$. Since within such an interval $\Delta t$ the wave may travel several rounds, therefore the real traveling angle can be $\Delta \theta_{0}=k \pi \pm \Delta \theta_{\text {min }}$. With the estimation from figure A.1 $T \simeq 0.045$, we know that $\Delta \theta_{0}=\pi / 3-\Delta \theta_{\min } \simeq 0.047998$. Rendered dimensionless by the rotation speed $\Omega_{i}$ of the inner cylinder, the wave rotation speed is then $\omega=\left|\frac{\Delta \theta_{0}}{\Delta t \Omega_{i}}\right| \simeq 0.34432$, which agrees well with the estimation. 

"He Who has overcome his FEARS Will truly BE FREE."

$$
\text { Aristotle (384 - } 322 \text { BC) }
$$

\section{B \\ ONSET OF SUSTAINED TURBULENCE IN LESS-RESOLVED COUETTE FLOW}

While direct numerical simulation of transition to turbulence at criticality requires significant amount of computing time to fully resolve the spatial and time scales, we resort to less-resolved simulations to make a reasonably good prediction with limited computing resources. This type of less-resolved simulations, as proposed and argued by Manneville [140], result in a downward shift in critical Reynolds number but preserves the qualitative features of transitional turbulence.

Simulations are carried out using a Petrov-Galerkin pseudospectral code [90]. The domain size is $\left(L_{z} \times L_{\theta} \times L_{r}\right)=(960 h \times 10 h \times 2 h)$, with a resolution $\left(n_{z} \times n_{\theta} \times n_{r}\right)=$ $(1276 \times 16 \times 12)$. Compared to the resolution for the fully resolved simulations, the current one is about 32 times lower, which reduces substantially the computational cost. This enables the simulations to run on a home cluster and therefore provides the possibility to investigate the dynamics at criticality and to check whether the transition to turbulence belongs to the DP universality class.

\section{B.1 Steady-state experiments}

We first performed a group of simulations to study the statistically stationary states. Simulations were done at different Reynolds number $R e \in[271.5,285]$, based on half 
Appendix B. Onset of sustained turbulence in less-resolved Couette flow

gap distance. Note that the lower bound for the critical Reynolds number at this resolutions is about 270 , determined by balancing the mean splitting time and lifetime of localized turbulent bands. At each Re, 20 realizations were conducted, with independent initial conditions. We did the same measurements of laminar gaps and other analysis as described in Chapter 33

Figure B.1 shows the temporal evolution of the ensemble-averaged (averaged over 20 independent realizations) turbulent fraction $F_{T}(t)$ at $R e=[271.75,272.5,273.5,275]$. Except for the case at $\operatorname{Re}=543.5$ which tends to decay, all other ones appears to reach a steady state with fluctuation around the mean. Moreover, the relaxation time depends on Re: The higher the Reynolds number is, the shorter the relaxation time becomes.

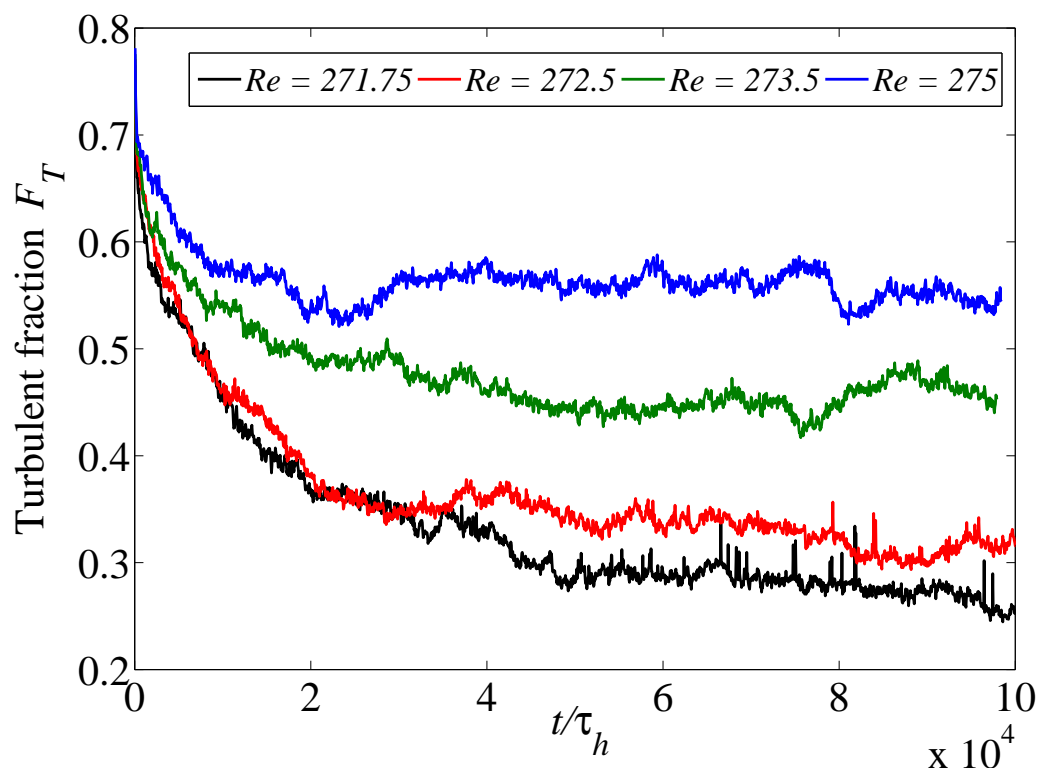

Figure B.1: Temporal evolution of the ensemble-averaged turbulent fraction at different Re. Each curve is obtained by averaging over 20 independent ones at the same Re. X axis is in advective time unit $\tau_{h}=h / U$.

In order to obtain the scaling relation with Re, the temporal mean $\bar{F}_{T}$ of the ensembleaveraged turbulent fraction is computed at the steady stage. The results are shown in Fig. B.2. The dashed line is the $\beta$-fit with the universal value $\beta=0.276$, giving a critical Reynolds number $R e_{c}^{\beta \text {-fit }} \simeq 271.9$. The fitting curve agrees very well with the simulation data. Note that the exponent $\beta$ from the best fit is about 0.25 .

The probability distributions (power density function) of the laminar gap sizes at Re close to the critical point follow a power-law scaling, while at higher Re the distributions are exponential, as shown in Fig. B.3. At Re $=271.75$, the fitting exponent of the tail of the distribution equals approximately -1.75 , which is consistent with the result from the fully-resolved simulations and the universal value in 1D-DP class. 


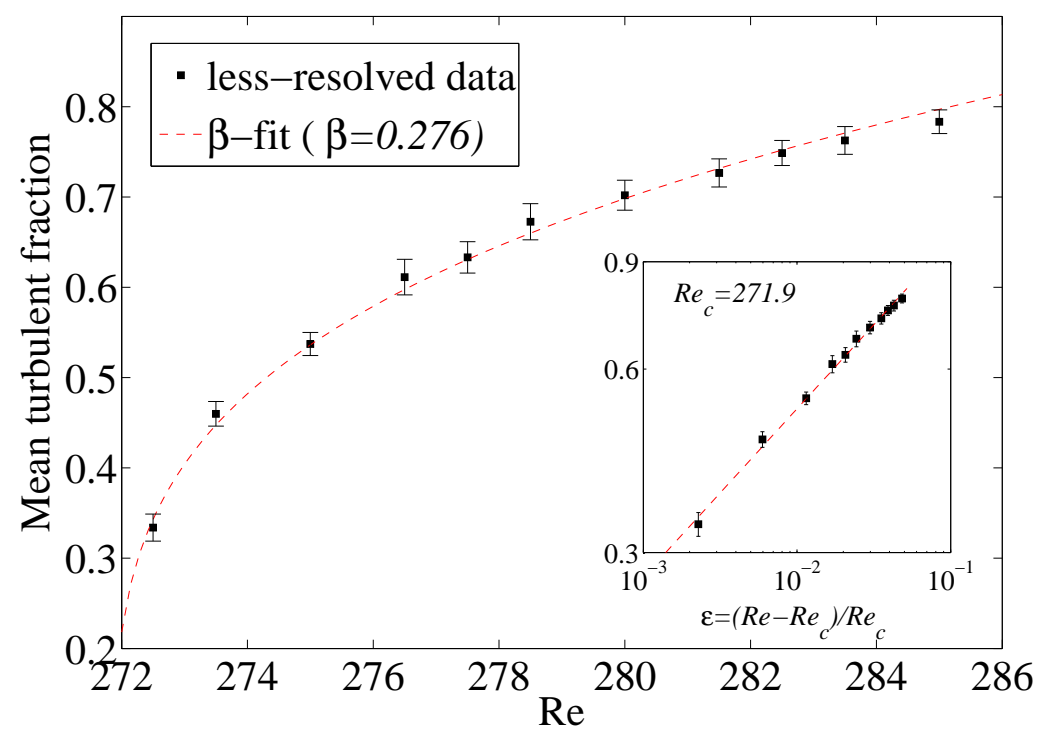

FiguRe B.2: Mean turbulent fraction as a function of Reynolds number. The red dashed line corresponds to the $\beta$-fit. Inset is the $\log$-log plot with $\epsilon=\frac{R e-R e_{c}}{R e_{c}}$ in X axis. The error bars are the standard deviation.

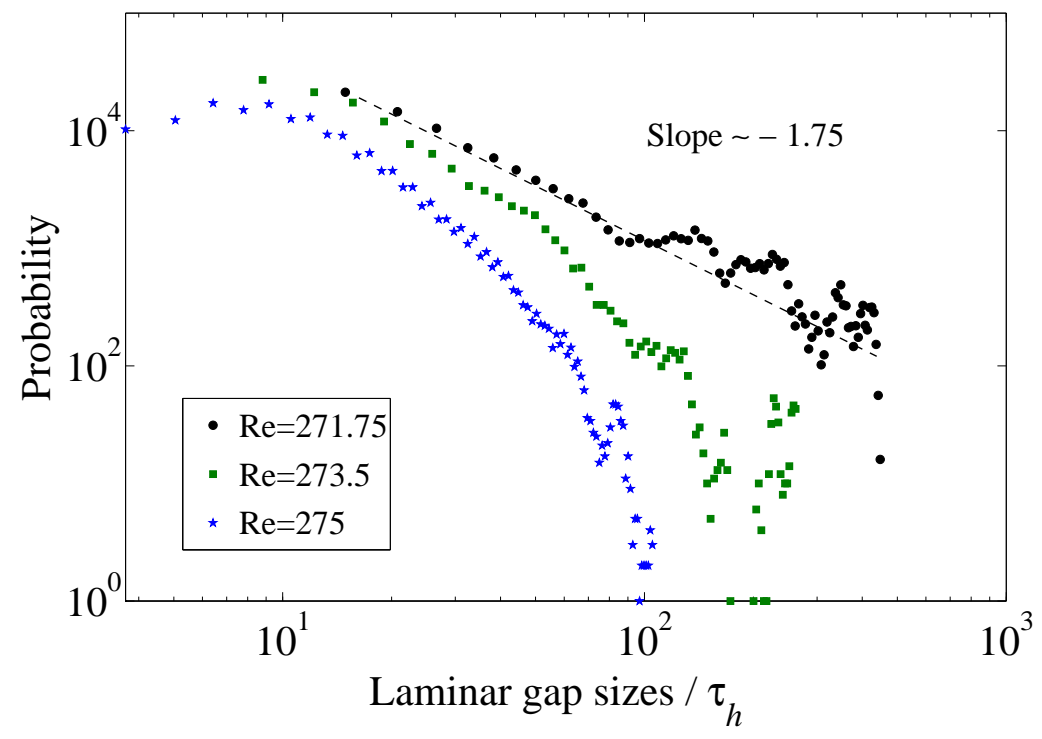

Figure B.3: Probability distributions (Power density function) of laminar gap sizes. The dashed line is the fitting curve to the black circle data set, at $R e=271.75$. Both axes are in logarithmic scale.

To confirm the DP universality class, a third exponent corresponding to the temporal correlation length is required. However, as stated in section $\$ 3.7$, it is rather difficult to obtain this exponent with steady-state experiments. An alternative method is to perform the quenching experiments, starting from a fully turbulent state obtained at very high Re and running at lower Re. The quenching experiments are described in the next section. 
Appendix B. Onset of sustained turbulence in less-resolved Couette flow

\section{B.2 Quenching experiments}

The initial conditions are obtained at $\operatorname{Re}=300$, with almost fully developed turbulence. The simulations were conducted at $R e \in[270,275]$. At criticality it is expected that the turbulent fraction decays algebraically as a power law, $F_{T}(t)=t^{-\alpha} F_{U}\left(\epsilon t^{1 / \nu_{\|}}\right)$with $\alpha=\beta / \nu_{\|}$and $F_{U}$ a universal scaling funtion. As Re goes away from the critical point, the turbulence decay or saturation within a finite duration. Our observation in Fig. B.4 confirms qualitatively the above scenario. With quenching experiments, the usual way to obtain the third exponent is to collapse all curves in Fig. B.4 by rescaling the axes, $t \rightarrow t\left|\epsilon^{\nu_{\|}}\right|$and $F_{T} \rightarrow F_{T} t^{\alpha}$ (see [51]). Since $\beta$ and the critical Reynolds number $R e_{c}$ are known from the steady-state experiments, we need to tune the third exponent such that the collapse of the curves is the best. To avoid the difficulty of defining a best collapse, in our case we simply choose the DP exponent $\nu_{\|}=1.734$ and check whether the curves collapse. The rescaled plot is shown in Fig. B.5, which resembles the one obtained from the systems in 1D-DP class (e.g. the turbulent liquid crystals in [55]). The good collapse supports that transition to turbulence in our system falls into the DP universality class.

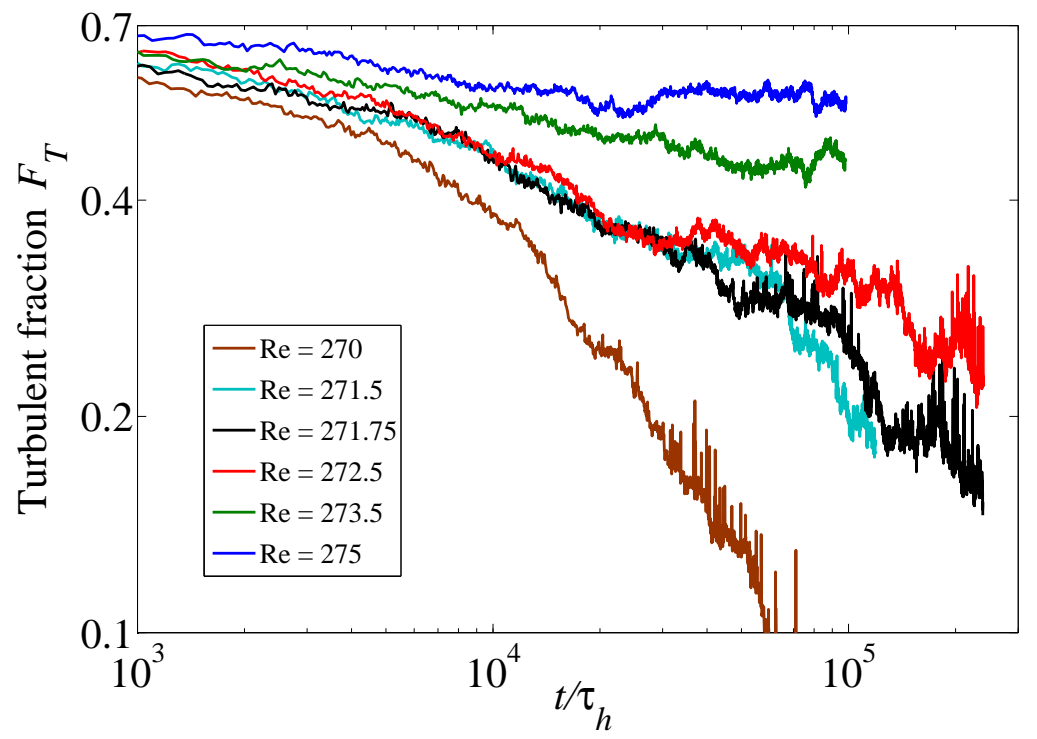

Figure B.4: Temporal evolution of the ensemble-averaged turbulent fraction in the quenching experiments at different Re. The axes are in logarithmic scale. It is shown that the evolution follow a power law close to the critical point.

\section{B.3 Discussion and conclusion}

For saving the computing time and obtaining preliminary insights into the dynamics at criticality, less-resolved simulations have been performed and three relevant critical 


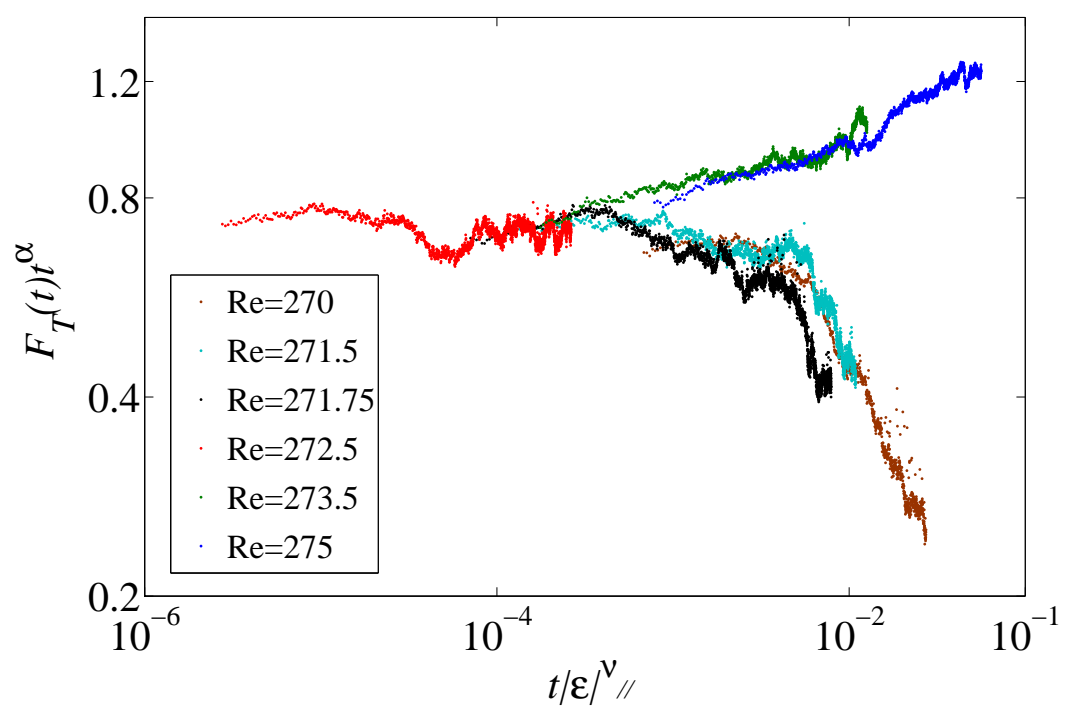

Figure B.5: The same date as in Fig. B.4 but with rescaled axes $t\left|\epsilon^{\nu_{\|}}\right|$and $F_{T} t^{\alpha}$.

exponents have been measured via steady-state and quenching experiments. The exponents are in good agreement with the DP universal value. These evidences show that the transition to turbulence in our less-resolved quasi-1D Couette flow is a continuous phase transition and falls into the DP universality class.

One may argue that less-resolved simulations are not a real physical system and the results presented here are not transferable to the real Couette flows. It is known that the less-resolved simulations results in a smaller critical Reynolds number. However, similar statistical results were obtained in both fully- or less-resolved systems. Figure B.6 shows the mean turbulent fraction as a function of the normalized Reynolds number $\epsilon$, a measure for the distance to the critical Re. The data points from both case fall on the same scaling relation (the $\beta$-fit). Note that the best fit of all the data points gives an exponent $\beta^{\text {best-fit }} \simeq 0.275$. These similarities indicate that in quenching experiments for the fully-resolved case we can have also the collapsed curves and obtain the DP temporal universal exponent. On the other hand, supposing that the quasi-1D Couette flow falls into the DP universality class, the less-resolved Couette flow should also belong to the DP class, since in the less-resolved system the localized turbulent stripes are preserved and they also split and decay with certain probabilities. Whether the smallest or microscopic structures inside turbulent stripes are resolved or not, it does not change the macroscopic qualitative behavior and hence may still belong to the DP universality class.

Another factor that may influence the results is the cutoff value of the vorticity averaged over the radial and azimuthal directions. Below this cutoff the flow is considered to be laminar. This influence is checked by varying the cutoff value, to see the change on the 
Appendix B. Onset of sustained turbulence in less-resolved Couette flow

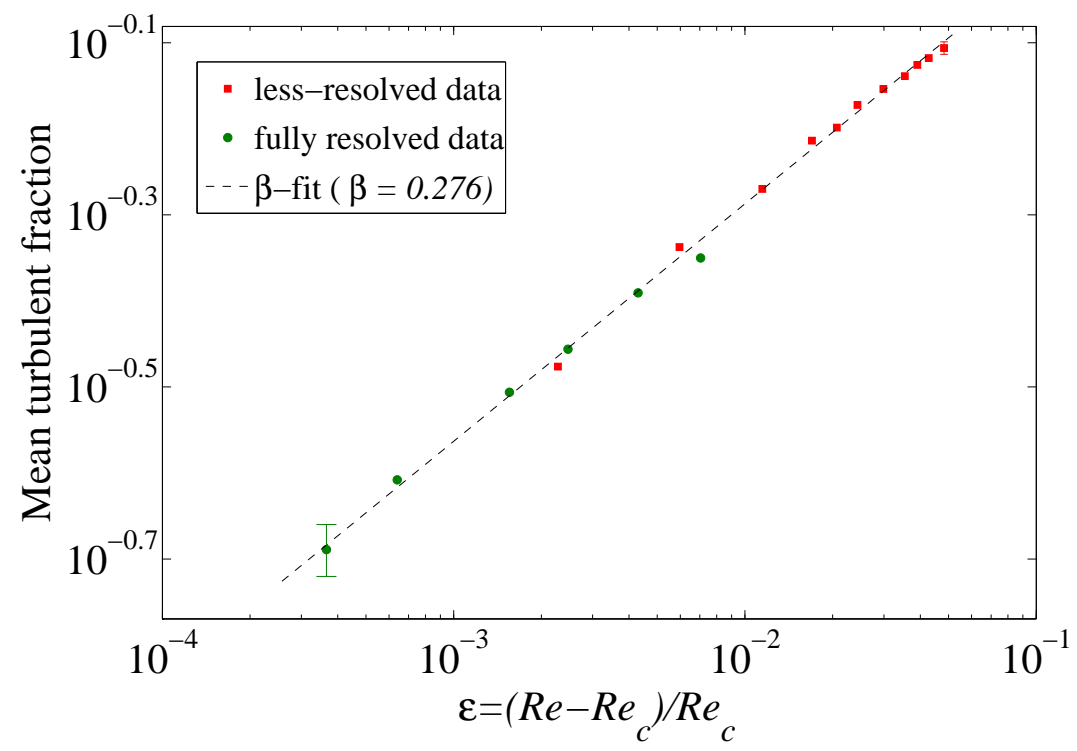

Figure B.6: Scaling of the mean turbulent fraction in both fully resolved (green dots) and less-resolved (red squares) simulations. The dashed line is the $\beta$-fit. In the best fit, $\beta \simeq 0.275$.

scaling relation of the mean turbulent fraction. Five different cutoff values are tested, ranging from $1 \times 10^{4}$ to $1 \times 10^{5}$. As is shown in Fig. B.7, higher cutoff value results in lower turbulent fraction. With an allowed range (not too high or too low), different cutoff values lead to a shift of the absolute value but the power exponent stays almost the same.

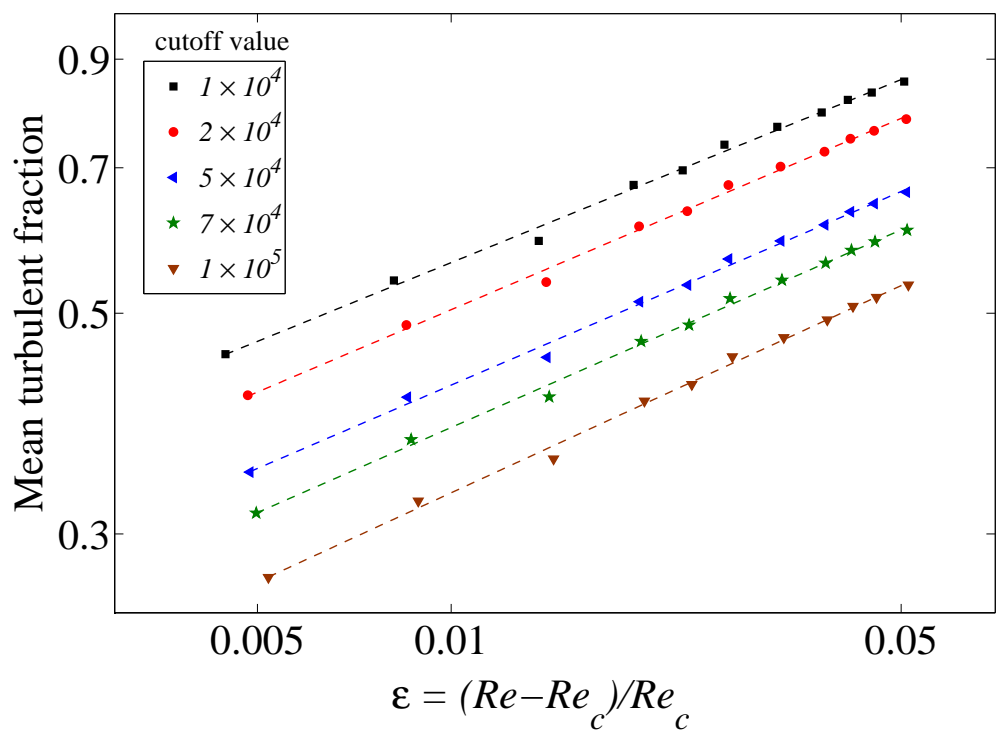

FiguRE B.7: Scalings of the mean turbulent fraction at different cutoff value of mean vorticity. Dashed lines are the best fits. 
"There IS ALWAYS A WELL-KNOWN SOLUTION TO EVERY HUMAN PROBLEM - NEAT, PLAUSible, AND WRONG."

Henry L. MENCKen (1880 - 1956)

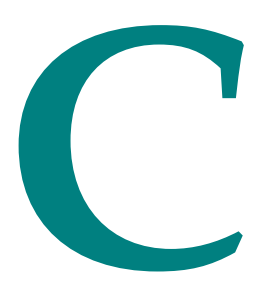

\section{BASE VELOCITY PROFILE IN EKMAN-COUETTE FLOW}

The base flow of plane Couette flow is known to be a linear profile, $\left(U_{0} y / 2,0,0\right)$. For the Ekman-Couette flow, the base flow is more complicated, including one more velocity component in $Z$ direction. We use here the coordinate system as shown in Fig. 5.1 , Because of the homogeneity in $X$ and $Z$ direction, the base profile depends only on $y$, as $\mathbf{U}(y)=(U(y), 0, W(y))$, which gives $\mathbf{U} \cdot \nabla \mathbf{U}=0$. The non-slip boundary conditions are chosen on the wall, namely, $U( \pm 1)= \pm U_{0} / 2 W$ and $W( \pm 1)=0$. Since the base flow is also independent of time, the equation 5.2 for the base flow can be expressed as

$$
\begin{aligned}
\frac{1}{\mathrm{Ro}} W & =-\partial_{x} P+\frac{1}{\mathrm{Re}} \partial_{y}^{2} U \\
0 & =-\partial_{y} P \\
-\frac{1}{\mathrm{Ro}} U & =-\partial_{z} P+\frac{1}{\mathrm{Re}} \partial_{y}^{2} W
\end{aligned}
$$

From Eq. C.2, we have $\partial_{x} \partial_{y} P=\partial_{z} \partial_{y} P=0$, which means that $\partial_{x} P$ and $\partial_{z} P$ are independent of $y$. Due to the sysmetry about the plane $y=0$, we obtain

$$
\left.\partial_{y}^{2} U\right|_{y=0}=\left.\partial_{y}^{2} W\right|_{y=0}=\left.U\right|_{y=0}=\left.W\right|_{y=0}=0
$$


Appendix C. Base velocity profile in Ekman-Couette flow

By evaluating Eq. C.1 and C.3 at $y=0$, it gives $\partial_{x} P=0$ and $\partial_{z} P=0$. Together with Eq. C.2, we know that the pressure $P$ of the base flow is constant everywhere. Therefore, the Eq. C.1 and C.3 are reduced as

$$
\begin{aligned}
\frac{1}{\mathrm{Ro}} W & =\frac{1}{\mathrm{Re}} \partial_{y}^{2} U \\
-\frac{1}{\mathrm{Ro}} U & =\frac{1}{\mathrm{Re}} \partial_{y}^{2} W
\end{aligned}
$$

Let us now introduce a complex function $Z(y)=U(y)+i W(y)$. By performing (C.5) $+\mathrm{i}(\overline{\mathrm{C} .6})$, we have

$$
Z^{\prime \prime}+i \frac{R e}{R o} Z=0
$$

, which is a second-order ordinary differential equation with boundary conditions. By assuming $Z=Z_{0} e^{i \gamma y}$, we have the eigen-equations of the ODE system, $-\gamma^{2}+i \frac{R e}{R o}=0$. The root of this equation is $\gamma_{ \pm}= \pm \sqrt{i \frac{R e}{R o}}= \pm \sqrt{\frac{R e}{R o}} \frac{1+i}{\sqrt{2}}$. Hence the general solution of Eq. C.7 is

$$
Z(y)=A_{+} e^{i \gamma_{+} y}+A_{-} e^{i \gamma_{-} y}, \quad \text { with } \gamma_{+}=-\gamma_{-} .
$$

The non-slip boundary conditions for $Z$ are

$$
\begin{aligned}
Z(1) & =\frac{U_{0}}{2}=A_{+} e^{i \gamma_{+}}+A_{-} e^{i \gamma_{-}} \\
Z(-1) & =-\frac{U_{0}}{2}=A_{+} e^{-i \gamma_{+}}+A_{-} e^{-i \gamma_{-}}
\end{aligned}
$$

By solving the linear equations, we obtain $A_{+}=A_{-}=\frac{U_{0}}{2} \frac{1}{e^{i \gamma_{+}}-e^{i \gamma_{-}}}$. The solution to Eq. C.7 can be thus writen as

$$
Z(y)=\frac{U_{0}}{2} \frac{e^{i \gamma y}-e^{-i \gamma y}}{e^{i \gamma}-e^{-i \gamma}}, \quad \text { with } \gamma=\sqrt{\frac{\operatorname{Re}}{\operatorname{Ro}}} \frac{1+i}{\sqrt{2}}
$$

Note that in non-dimensional form $U_{0}=1$. In summary, the base velocity of the Ekman-Couette flow is $[U(y), V(y), W(y)]=[\operatorname{Re}(Z), 0, \operatorname{Im}(Z)]$. 
"IF I HAVE SEEN FARTHER THAN OTHERS, IT IS BY STANDING ON THE SHOULDERS OF GIANTS."

Sir IsaAC Newton (1642 - 1727)

"If I haVe not SEen AS far as Others, IT IS BECAUSE GIANTS WERE STANDING ON MY SHOULDERS."

Harold (Hal) Abelson (1947 - )

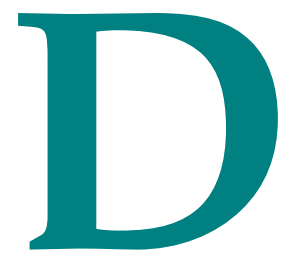

\section{PublicAtions}

1. L. Shi, M. Avila, B. Hof,

Scale Invariance at the Onset of Turbulence in Couette Flow,

Phys. Rev. Lett., 110, 204502 (2013).

2. L. Shi, B. Hof, A. Tilgner,

Transient Growth of Ekman-Couette Flow,

Phys. Rev. E, 89, 013001 (2014).

3. L. Shi, M. Rampp, B. Hof, M. Avila,

A Hybrid MPI-OpenMP Parallel Implementation for Pseudospectral Simulations: Application to Taylor-Couette Flow, accepted with minor revision to Computers $\&$ Fluids, (2014). 



\section{BIBLIOGRAPHY}

[1] O. Reynolds. An experimental investigation of the circumstances which determine whether the motion of water shall be direct or sinuous, and of the law of resistance in parallel channels. Proc. R. Soc. Lond., 35:84-99, 1883.

[2] Lord Rayleigh. On the dynamics of revolving fluids. Proc. R. Soc. Lond. A, 93: 148-154, 1917.

[3] G. I. Taylor. Stability of a viscous liquid contained between two rotating cylinders. Proc. R. Soc. Lond. A, 223:289-343, 1923.

[4] G. I. Taylor. Fluid friction between rotating cylinders. I. torque measurements. Proc. R. Soc. Lond. A, 157:546-564, 1936.

[5] L. D. Landau. On the problem of turbulence. CR Acad. Sci. URSS, 44:1-314, 1944.

[6] J. Rotta. Experimenteller beitrag zur entstehung turbulenter strömung im rohr. Arch. Appl. Mech., 24:258-281, 1956.

[7] E. N. Lorenz. Deterministic nonperiodic flow. Journal of the Atmospheric Sciences, 20:130-141, 1963.

[8] Donald Coles. Transition in circular couette flow. J. Fluid Mech., 21:385-425, 1965.

[9] D. Ruelle and F. Takens. On the nature of turbulence. Commun. Math. Phys., 20:167-192, 1971.

[10] H. L. Swinney and J. P. Gollub. The transition to turbulence. Phys. Today, 31: 41, 1978.

[11] Y. Pomeau. Front motion, metastability and subcritical bifurcations in hydrodynamics. Physica D, 23:3-11, 1986.

[12] K. Avila, D. Moxey, A. D. Lozar, M. Avila, D. Barkley, and B. Hof. Onset of sustained turbulence in pipe flow. Science, 333:192, 2011. 
[13] Kerstin Avila. Experiments on shear flows: Characterizing the onset of turbulence as a phase transition. $\mathrm{PhD}$ thesis, Georg-August-Universität Göttingen, Germany, 2013.

[14] C. D. Andereck, S. S. Liu, and H. L. Swinney. Flow regimes in a circular couette system with independently rotating cylinders. J. Fluid Mech., 164:155-183, 1986.

[15] B. Hof, A. Juel, and T. Mullin. Scalint of the turbulence transition threshold in a pipe. Phys. Rev. Lett., 91:244502, 2003.

[16] Björn Hof, Jerry Westerweel, Tobias M. Schneider, and Bruno Eckhardt. Finite lifetime of turbulence in shear flows. Nature, 443:59-62, 2006.

[17] Bruno Eckhardt, Tobias M. Schneider, Bjrn Hof, and Jerry Westerweel. Turbulence transition in pipe flow. Ann. Rev. Fluid Mech., 39:447-468, 2007.

[18] Marc Avila, Ashley P. Willis, and Björn Hof. On the transient nature of localized pipe flow turbulence. J. Fluid Mech., 646:127-136, 2010.

[19] T. Mullin. Experimental studies of transition to turbulence in a pipe. Ann. Rev. Fluid Mech., 43:1-24, 2011.

[20] K. Avila, D. Moxey, A. D. Lozar, M. Avila, D. Barkley, and B. Hof. Onset of sustained turbulence in pipe flow. Science, 333(192), 2011.

[21] V. A. Romanov. Stability of plane-parallel couette flow. Funct. Anal. Appl., 7: 137-146, 1973.

[22] P. G. Drazin and W. H. Reid. Hydrodynamics Stability. Cambridge university press, 1981.

[23] Nils Tillmark and P. Henrik Alfredsson. Experiments on transition in plane couette flow. J. Fluid Mech., 235:89-102, 1992.

[24] Anders Lundbladh and Arne V.Johansson. Direct simulation of turbulent spots in plane couette flow. J. Fluid Mech., 229:499-516, 1991.

[25] J. P. Gollub and H. L. Swinney. Onset of turbulence in a rotating fluid. Phys. Rev. Lett., 35:927-930, 1975.

[26] Hugues Chaté and Paul Manneville. Transition to turbulence via spatiotemporal intermittency. Phys. Rev. Lett., 58:112-115, 1987.

[27] A. N. Kolmogorov. The local structure of turbulence in incompressible viscous fluid for very large reynolds numbers. Proc. R. Soc. Lond. A, 434:9-13, 1991.

[28] A. N. Kolmogorov. Dissipation of energy in the locally isotropic turbulence. Proc. R. Soc. Lond. A, 434:15-17, 1991. 
[29] G. Karniadakis and S. Orszag. Nodes, modes and flow codes. Phys. Today, pages 34-42, March 1993.

[30] Holger Faisst and Bruno Eckhardt. Transition from couette-taylor system to the plane couette system. Phys. Rev. E, 61:7227, 2000.

[31] Lord Kelvin. Stability of fluid motion-rectilinear motion of viscous fluid between two parallel planes. Philos Mag., 24:188-197, 1887.

[32] L. Hopf. Verlauf kleiner schwingungen auf einer strmung reibender flssigkeit. Ann. Phys., 349:1, 1914.

[33] W. H. Reid. Uniform asymptotic approximations to the solutions of the orrsommerfeld-equation. part 1. plane couette flow. Stud. Appl. Math., 53:91, 1974.

[34] K. M. Case. Stability of inviscid plane couette flow. Phys. Fluids, 3:143-148, 1960.

[35] T. Ellingsen and E. Palm. Stability of linear flow. Phys. Fluids, 18:487-488, 1974.

[36] M. Nagata. Three-dimensional finite-amplitude solutions in plane couette flow: bifurcation from infinity. J. Fluid Mech., 217:519-527, 1990.

[37] F. Daviaud, J. Hegseth, and P. Bergé. Subcritical transition to turbulence in plane couette flow. Phys. Rev. Lett., 69:2511-2514, 1992.

[38] O. Dauchot and F. Daviaud. Finite amplitude perturbation and spots growth mechanism in plane couette flow. Phys. Fluids, 7:335-343, 1995.

[39] S. Bottin and H. Chaté. Statistical analysis of the transition to turbulence in plane couette flow. Eur. Phys. J. B, pages 143-155, 1998.

[40] P. Moin and J. Kim. Tackling turbulence with supercomputers. Scientific American, 276:62-68, January 1997.

[41] P. Moin and K. Mahesh. Direct numerical simulation: A tool in turbulence research. Ann. Rev. Fluid Mech., 30:539-578, 1998.

[42] J. Jiménez. Computing high-reynolds-number turbulence: will simulations ever replace experiments? J. of Turbulence, 4:1-13, 2003.

[43] Y. Duguet, P. Schlatter, and D. S. Henningson. Formation of turbulent patterns near the onset of transition in plane couette flow. J. Fluid Mech., 650:119-129, 2010. 
[44] Tobias M. Schneider, Filippo De Lillo, Juergen Buehrle Bruno Eckhardt, Tim Dörnemann, Kay Dörnemann, and Bernd Freisleben. Transient turbulence in plane couette flow. Phys. Rev. E, 81(025301), 2010.

[45] Björn Hof, Alberto de Lozar, Dirk Jan Kuik, and Jerry Westerweel. Repeller or attractor? selecting the dynamical model for the onset of turbulence in pipe flow. Phys. Rev. Lett., 101:214501, 2008.

[46] I. J. Wygnanski and F. H. Champagne. On transition in a pipe. part 1. the origin of puffs and slugs and the flow in a turbulent slug. J. Fluid Mech., 59:281-335, 1973.

[47] Arnaud Prigent, Guillaume Grégoire, Hugues Chaté, Olivier Dauchot, and Wim van Saarloos. Large-scale finite-wavelength modulation within turbulent shear flow. Phys. Rev. Lett., 89:014501, 2002.

[48] Dwight Barkley and Laurette S. Tuckerman. Computational study of turbulent laminar patterns in couette flow. Phys. Rev. Lett., 94(014502), 2005.

[49] S. R. Broadbent and J. M. Hammersley. Percolation processes i. crystals and mazes. Proc. Cambr. Phil. Soc., 53:629-641, 1957.

[50] S. P. Obukhov. The problem of directed percolation. Physica, 101A:145-155, 1980.

[51] Malte Henkel, Haye Hinrichsen, and Sven Lübeck. Non-Equilibrium Phase Transitions - Volume I: Absorbing Phase Transitions. Springer, 2008.

[52] Kunihiko Kaneko. Spatiotemporal intermittency in coupled map lattices. Progr. Theoret. Phys., 74:1033-1044, 1985.

[53] Hans Karl Janssen. On the non-equilibrium phase-transition in reaction- diffusion systems with an absorbing stationary state. Z. Phys. B: Cond. Mat., 42:151-154, 1981.

[54] P. Grassberger. On phase transition in schlögl's second model. Z. Phys. B: Cond. Mat., 47:365-374, 1982.

[55] K. A. Takeuchi, M. Kuroda, H. Chaté, and M. Sano. Directed percolation criticality in turbulent liquid crystals. Phys. Rev. Lett., 99(234503), 2007.

[56] H. Hinrichsen. Nonequilibrium critical phenomena and phase-transitions into absorbing states. Adv. Phys., 49:815-958, 2000.

[57] Hugues Chaté and Paul Manneville. Spatio-temporal intermittency in coupled map lattices. Physica D, 32:409-422, 1988. 
[58] S. Bottin, F. Daviaud, P. Manneville, and O. Dauchot. Discontinuous transition to spatiotemporal intermittency in plane couette flow. Europhys. Lett., pages 171-176, 1998.

[59] Paul Manneville. Spatiotemporal perspective on the decay of turbulence in wallbounded flows. Phys. Rev. E, 79:025301(R), 2009.

[60] David Moxey and Dwight Barkley. Distinct large-scale trubulent-larminar states in transitional pipe flow. Proc. Natl. Acad. Sci. U.S.A., 107(18):8091-8096, 2010.

[61] Satish C. Reddy Lloyd N. Trefethen, Anne E. Trefethen and Tobin A. Driscoll. Hydrodynamic stability without eigenvalues. Science, 261:579-584, 1993.

[62] Peter J. Schmid and Dan S. Henningson. Stability and Transition in Shear Flows. Springer, 2001.

[63] Peter J. Schmid. Nonmodal stability theory. Ann. Rev. Fluid Mech., 39:129-162, 2007.

[64] N. I. Shakura and R. A. Sunyaev. Black holes in binary systems. observational appearance. Astron. Astrophys., 24:337-355, 1973.

[65] D. N. C. Lin and J. C. B. Papaloizou. Theory of accretion disks ii: Application to observed systems. Annu. Rev. Astron. Astrophys., 34:703-747, 1996.

[66] John S. Carr, Alan T. Tokunaga, and Joan Najita. Hot h2o emission and evidence for turbulence in the disk of a young star. Astrophys. J., 603:213-220, 2004.

[67] A. Meredith Hughes, David J. Wilner, Sean M. Andrews, Chunhua Qi, and Michiel R. Hogerheijde. Empirical constraints on turbulence in protoplanetary accretion disks. Astrophys. J., 727:85, 2011.

[68] Steven A. Balbus and John F. Hawley. A powerful local shear instability in weakly magnetized disks. i. linear analysis. Astron. Astrophys., 376:214-222, 1991.

[69] Steven A. Balbus and John F. Hawley. Instability, turbulence, and enhanced transport in accretion disks. Rev. Mod. Phys., 70:1-53, 1998.

[70] Steven A. Balbus. Enhanced angular momentum transport in accretion disks. Annu. Rev. Astron. Astrophys., 41:555-597, 2003.

[71] Denis Richard and Jean-Paul Zahn. Turbulence in differentially rotating flows. what can be learned from the couette-taylor experiment. Astron. Astrophys., 347:734, 1999. 
[72] H. Ji, M. J. Burin, E. Schartman, and J. Goodman. Hydrodynamic turbulence cannot transport angular momentum effectively in astrophysical disks. Nature, 444:343-346, 2006.

[73] E. Schartman, H. Ji, M. J. Burin, and J. Goodman. Stability of quasi-keplerian shear flow in a laboratory experiment. Astron. Astrophys., 543:A94, 2012.

[74] M. S. Paoletti, D. P. M. van Gils, B. Dubrulle, C. Sun, D. Lohse, and D. P. Lathrop. Angular momentum transport and turbulence in laboratory models of keplerian flows. Astron. Astrophys., 547:A64, 2012.

[75] M. Avila. Stability and angular-momentum transport of fluid flows between corotating cylinders. Phys. Rev. Lett., 108(124501), 2012.

[76] Y. B. Zeldovich. On the friction of fluids between rotating cylinders. Proc. R. Soc. Lond. A, 374:299-312, 1981.

[77] F. Hersant, B. Dubrulle, and J.-M. Huré. Turbulence in circumsteller disks. Astron. Astrophys., 429:531-542, 2005.

[78] S. Balbus. A turbulent matter. Nature, 470:475-476, 2011.

[79] Steven A. Orszag and G. S. Patterson. Numerical simulation of three-dimensional homogeneous isotropic turbulence. Phys. Rev. Lett., 28:76-79, 1972.

[80] P. R. Spalart. Direct simulation of a turbulent boundary layer up to $r_{\theta}=1410$. J. Fluid Mech., 187:61-98, 1988.

[81] P. Schlatter and R. Örlü. Assessment of direct numerical simulation data of turbulent boundary layers. J. Fluid Mech., 659:116-126, 2010.

[82] J. Kim, P. Moin, and R. Moser. Turbulence statistics in fully developed channel flow at low reynolds number. J. Fluid Mech., 177:133-166, 1987.

[83] S. Hoyas and J. Jiménez. Scaling of the velocity fluctuations in turbulent channels up to $r e_{\tau}=2003$. Phys. Fluids, 18(011702), 2006.

[84] J.G.M. Eggels, F. Unger, M.H. Weiss, J. Westerweel, R.J. Adrian, R. Friedrich, and F.T.M. Nieuwstadt. Fully developed turbulent pipe flow: a comparison between direct numerical simulation and experiment. J. Fluid Mech., 268:175209, 1994.

[85] X. Wu, J. R. Baltzer, and R. J. Andrian. Direct numerical simulation of a 30r long turbulent pipe flow at $r^{+}=685$ : large- and very large-scale motions. J. Fluid Mech., 698:235-281, 2012.

[86] K. Coughlin and P.S. Marcus. Turbulent bursts in couette-taylor flow. Phys. Rev. Lett, 77 (11):2214-2217, 1996. 
[87] H. J. Brauckmann and B. Eckhardt. Direct numerical simulation of local and global torque in taylor-couette flow up to $r e=30000$. J. Fluid Mech., 718: 398-427, 2013.

[88] Stephen B. Pope. Turbulent Flows. Cambridge University Press, 2000.

[89] R. D. Moser, P. Moin, and A. Leonard. A spectral numerical method for the navier-stokes equations with applications to taylor-couette flow. J. Comput. Phys., 52:524-544, 1983.

[90] A. Meseguer, Marc Avila, F. Mellibovsky, and F. Marques. Solenoidal spectral formulations for the computation of secondary flows in cylindrical and annular geometries. Eur. Phys. J. Special Topics, 146:249-259, 2007.

[91] Philip S Marcus. Simulation of taylor-couette flow. part 1. numerical methods and comparison with experiment. Journal of Fluid Mechanics, 146(1):45-64, 1984.

[92] Dan Kasloff and Hillel Tal-Ezer. A modified chebyshev pseudospectral method with an $\mathcal{O}\left(n^{-1}\right)$ time step restriction. J. Comput. Phys., 104:457-469, 1993.

[93] S. Hugues and A. Randriamampianina. An improved projection scheme applied to pseudospectral methods for the imcompressible navier-stokes equations. Int. J. Numer. Meth. Fluids, 28:501-521, 1998.

[94] P. M. Gresho and R. L. Sani. On pressure boundary conditions for the incompressible navier-stokes equations. Int. J. Numer. Methods Fluids, 7:1111-1145, 1987.

[95] S. A. Orszag and A. T. Patera. Secondary instability of wall-bounded shear flows. J. Fluid Mech., 128:347-385, 1983.

[96] Bengt Fornberg. A pratical guide to pseudospectral methods. Cambridge university press, 1998.

[97] G. E. Karniadakis, M. Israeli, and S. A. Orszag. High-order splitting methods for the incompressible navier-stokes equations. J. Comput. Phys., 97:414-443, 1991.

[98] J. Dongarra, P. Beckman, et al. The international exascale software project roadmap. International Journal of High Performance Computer Applications, 25(1):3-60, 2011. ISSN 1094-3420.

[99] Matteo Frigo and Steven G. Johnson. The design and implementation of FFTW3. Proceedings of the IEEE, 93(2):216-231, 2005. Special issue on "Program Generation, Optimization, and Platform Adaptation". 
[100] D. Pirró and M. Quadrio. Direct numerical simulation of turbulent taylor-couette flow. Eur. J. Mech. B/Fluids, 27:552-566, 2008.

[101] Gregory P. King, Y. Li, W. Lee, Harry L. Swinney, and Philip S. Marcus. Wave speeds in wavy taylor-vortex flow. J. Fluid Mech., 141:365-390, 1984.

[102] Isabelle Raspo, Sandrine Hugues, Eric Serre, Anthony Randriamampianina, and Patrick Bontoux. A spectral projection method for the simulation of complex three-dimensional rotating flows. Computers \& fluids, 31:745-767, 2002.

[103] L. Shi, M. Avila, and B. Hof. Scale invariance at the onset of turbulence in couette flow. Phys. Rev. Lett., 110(204502), 2013.

[104] Simon Maretzke, Björn Hof, and Marc Avila. Transient growth in linearly stable taylor-couette flows. J. Fluid Mech., 742:254-290, 2014.

[105] Daniel Borrero-Echeverry, Michael F. Schatz, and Randall Tagg. Transient turbulence in taylor-couette flow. Phys. Rev. E, 81(025301), 2010.

[106] Liang Shi. Onset of sustained turbulence in narrow-gap taylor-couette flow. Master's thesis, École Polytechnique, France, 2010.

[107] Laurette S. Tuckerman and Dwight Barkley. Patterns and dynamics in transitional plane couette flow. Phys. Fluids, 23:041301, 2011.

[108] J.F. Lawless. Statistical Models and Methods for Lifetime Data. Wiley, New Jersey, second edition, 2003.

[109] Paul Manneville. Turbulent patterns in wall-bounded flows: a turing instability? Europhys. Lett., 98:64 001, 2012.

[110] B. Dubruelle, O. Dauchot, F. Daviaud, P-Y. Longaretti, D. Richard, and J-P. Zahn. Stability and turbulent transport in rotating shear flow: prescription from analysis of cylindrical and plane couette flows data. Phys. Fluids, 17:095103, 2005 .

[111] Rodolfo O. Mónico, Roberto Verzicco, Siegfried Grossman, and Detlef Lohse. Turbulence decay towards the linearly-stable regime of taylor-couette flow. arXiv:1311.2463, 2013.

[112] Eric M. Edlund and Hantao Ji. Nonlinear stability of laboratory quasi-keplerian flows. Phys. Rev. E, 89:021004(R), 2014.

[113] P. J. Armitage. Dynamics of protoplanetary disks. Ann. Rev. Astron. Astrophys., 49:195-236, 2011. 
[114] Mark R. Petersen, Keith Julien, and Glen R. Stewart. Baroclinic vorticity production in protoplanetary disks. i. vortex formation. Astrophys. J., 658:12361251, 2007.

[115] Mark R. Petersen, Glen R. Stewart, and Keith Julien. Baroclinic vorticity production in protoplanetary disks. i. vortex formation. Astrophys. J., 658:12521263, 2007.

[116] Mark R. Petersen, Glen R. Stewart, and Keith Julien. The subcritical baroclinic instability in local accretion disc models. Astron. Astrophys., 513:A60, 2010.

[117] F. Rincon, G. I. Ogilvie, and C. Cossu. On self-sustaining processes in rayleighstable rotating plane couette flows and subcritical transition to turbulence in accretion disks. Astron. Astrophys., 463:817-832, 2007.

[118] T. Tsukahara, N. Tillmark, and P. H. Alfredsson. Flow regimes in a plane couette flow with system rotation. J. Fluid Mech., 648:5-33, 2010.

[119] N. Hoffmann, F. H. Busse, and W. L. Chen. Transitions to complex flows in the ekman-couette layer. J. Fluid Mech., 366:311-331, 1998.

[120] Y. Ponty, A. D. Gilbert, and A. M. Soward. The onset of thermal convection in ekman-couette shear flow with oblique rotation. J. Fluid Mech., 487:91-123, 2003.

[121] A. A. Draad and F. T. M. Nieuwstadt. The earth's rotation and laminar pipe flow. J. Fluid Mech., 361:297-308, 1998.

[122] Eric Brown and Guenter Ahlers. Effect of the earth's coriolis force on the large-scale circulation of turbulent rayleigh-bérnard convection. Phys. Fluids, 18:125108, 2006.

[123] J. Boisson, D. Cébron, F. Moisy, and P. P. Cortet. Earth rotation prevents exact solid-body rotation of fluids in the laboratory. EPL, 98:59002, 2012.

[124] D. P. Lathrop S. A. Triana, D. S. Zimmerman. Precessional states in a laboratory model of the earth's core. J. Geophys. Res. : Solid Earth, 117:B04103, 2012.

[125] M. S. Paoletti and D. P. Lathrop. Angular momentum transport in turbulent flow between independently rotating cylinders. Phys. Rev. Lett., 106(024501), 2011.

[126] Satish C. Reddy and Dan S. Henningson. Energy growth in viscous channel flows. J. Fluid Mech., 252:209-238, 1993.

[127] Steven A. Orszag and Lawrence C. Kells. Transition to turbulence in plane poiseuille and plane couette flow. J. Fluid Mech., 96:159-205, 1980. 
[128] P. G. Drazin and W. H. Reid. Hydronamic Stability. Cambridge University Press, 1981.

[129] Douglas K. Lilly. On the instability of ekman boundary flow. J. Atmos. Sci., 23: 481-494, 1966.

[130] Kathryn M. Butler and Brian F. Farrell. Three-dimensional optimal perturbations in viscous shear flow. Phys. Fluids A, 4:1637-1650, 1992.

[131] William H. Press, Saul A. Teukolsky, William T. Vetterling, and Brain P. Flannery. Numerical Recipes: The Art of Scientific Computing. Cambridge University Press, 2007.

[132] E. M. Aydin and H. J. Leutheusser. Plane-couette flow between smooth and rough walls. Experiments in Fluids, 11:302-312, 1991.

[133] Dominik Krug, Beat Lüthi, Hansjörg Seybold, Markus Holzner, and Arkady Tsinober. 3d-ptv measurements in a plane couette flow. Exp. Fluids, 52:1349$1360,2012$.

[134] D. Barkley. Simplifying the complexity of pipe flow. Phys. Rev. E, 84(016309), 2011.

[135] Dwight Barkley and Laurette S. Tuckerman. Mean flow of turbulent-larminar patterns in plane couette flow. J. Fluid Mech., 576:109-137, 2007.

[136] Yohann Duguet and Philipp Schlatter. Oblique laminar-turbulent interfaces in plane couette flows. Phys. Rev. Lett., 110:034502, 2013.

[137] Gordon I. Ogilvie and Henrik N. Latter. Local and global dynamics of warped astrophysical discs. Mon. Not. R. Astro. Soc., 443:2403-2419, 2013.

[138] Gordon I. Ogilvie and Henrik N. Latter. Hydrodynamic instability in warped astrophysical discs. Mon. Not. R. Astro. Soc., 443:2420-2435, 2013.

[139] Richard P. Brent. Algorithms for Minimization without Derivatives. Dover Publications, 2002.

[140] Paul Manneville and J. Rolland. On modelling transitional turbulent flows using under-resolved direct numerical simulations: the case of plane couette flow. Theor. Comput. Fluid Dyn., 25:407-420, 2011. 